

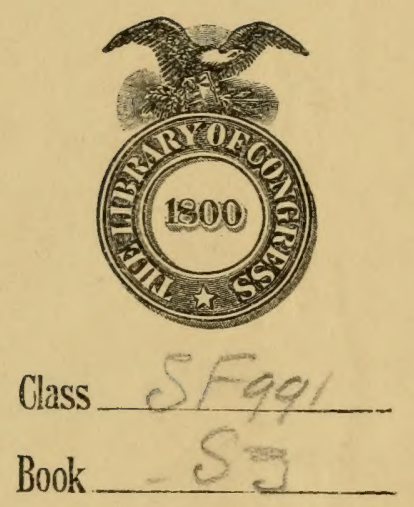

Copyight №.

COPYRIGHT DEPOSIT. 


$-2=$

is 



\section{VETERINARY MEDICINE SERIES \\ No. 5}

Edited by D. M. CAMPBELL, D. V. S.

\section{CANINE MEDICINE AND SURGERY}

BY

CHAS. G. SAUNDERS, V.S., B. V.Sc.

Senior Professor Ontario Veterinary College, Professor

Canine and Feline Medicine Ontario Veterinary

College, Editor Canine Department Amer-

ican Journal of Veterinary Medicine, O. C. No. 2 Sect. Canadian

ARMY VeTERINARY

CoRPS, Etc.

Chicago

AMERICAN JOURNAL OF VETERINARY MEDICINE 1915 


\section{SF991 \\ .53}

Copyright, 1915 ,

BY

D. M. Campbell

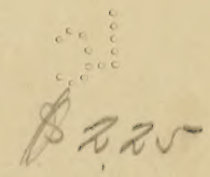

MAR -31915

(C) Cl. A 393882

no, 


\section{PREF A C E}

$\mathrm{I}^{\mathrm{N}}$ offering this little work to the profession the I author wishes to point out that it is publisher mainly for the use of senior students and practitioners. It presumes a knowledge of pathology, histology and anatomy, and aims to deal only with the clinical aspect of the various diseases. It does not presume to be an encyclopedia, but deals merely with the conditions commonly found in everyday practice. If it succeeds in arousing more interest in and deeper study of canine practice, the author feels that his labor will not have been in vain. Toronto, Ont.

C. G. S. 


\section{SENATOR VEST'S EULOGY ON THE DOG}

The best friend a man has in this world may turn against him and become his enemy. His son and daughter that he has reared with loving care may become ungrateful. Those who are nearest and dearest to us, those whom we trust with our happiness and our good name, may become traitors to their faith. The money that a man has he may lose. It flies away from him when he may need it most. Man's reputation may be sacrificed in a moment of ill-considered action. The people who are prone to fall on their knees and do us honor when success is with us may be the first to throw the stone of malice when failure settles its cloud upon our heads. The one absolutely unselfish friend a man may have in this selfish world, the one that never deserts him, the one that never proves ungrateful or treacherous, is the dog.

A man's dog stands by him in prosperity and poverty, in health and in sickness. He will sleep on the cold ground, when the winiry winds blow and the snow drives fiercely, if only he may be near his master's side. He will kiss the hand that has no food to offer, he will lick the wounds and sores that come in encounter with the roughness of the world. He guards the sleep of his pauper masier as if he were a prince.

When all o'her friends desert, he remains. When riches take wings and reputation falls to pieces he is as constant in his love as the sun in its journey through the heavens. If fortune drives the master forth an outcast into the world, friendless and homeless, the faithful dog asks no higher privilege than that of accompanying him, to guard him against danger, to fight against his enemies, and when the last scene of all comes and death takes his master in its embrace and his body is laid away in the cold ground, no matter if all other friends pursue their way, there by his graveside will the noble dog be found, his head between his paws and his eyes sad, but open in alert watchfulness, faithful and true even to death. 


\section{CONTENTS}

\section{PART I}

SECTION I.

Gexerif, ReMirks

SECTION II.

Diseases of the Upper Respiratory Passages..........

Simple Nasal Catarrh - Rhinitis - Coryza-Epistaxis-Laryngitis-Chronic Laryngitis.

\section{SECTION III.}

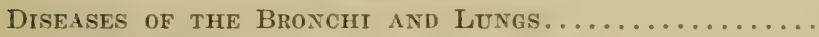

Bronchitis-Acute Bronchitis-Chronic Bronchitis -Pneumonia-Lobar (Croupous) Pneumonia-Lobular (Catarrhal) Pneumonia.

SECTION IV.

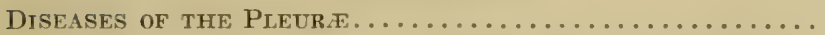

Pleurisy-Inflammation of the Pleura; PleuritisHydrothorax (Dropsy of the Thoracic Cavity)Pleurodynia.

\section{SECTION V.}

Diseases of the Mouth, Pharynx axd Esophagus.....

Stomatitis-Stomatitis Ulcerosa or Necrotic Stomatitis-Glossitis-Ranula-Epulis-Papilloma or Wart - Neoplasms-Harelip and Cleft Palate-The TeethTartar-Caries of the Teeth (Caries Dentum)-Empyema of the Superior Maxillary Sinus-Pharyngitis, or Sore Throat-Parotitis; Inflammation of the Parotid Gland (Mumps).

\section{SECTION VI.}

Diseases of the Stomach and Intestixes...........

Impaction of the Stomach-Gastritis-Acute Gastritis-Chronic Gastritis-Foreign Bodies in the Stomach-Intestinal Catarrh-Colic-Intussusception -Diarrhea-Constipation-Typhilitis-Enteritis.

\section{SECTION VII.}

DIseases of the Rectum AND ANus..............

Hemorrhoids-Prolapsus Recti-Amputation of the Prolapsed Rectum-Prolapsus Ani-Fistula in AnoAtresia Ani-Obstruction of the Anal Glands. 
SECTION VIII.

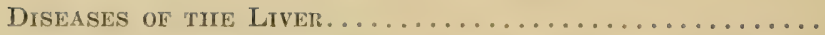

Icterus or Jaundice-Catarrhal Jaundice-Heptogenous Icterus-Hematogenous Icterus-Malignant Malarial Jaundice-Hepatitis; Inflammation of the Liver-Cirrhosis of the Liver; Interstitial Hepatitis - Ascites or Abdominal Dropsy-Diabetes Mellitus.

SECTION IX.

DISLASES OF THE KIDNEY . . . . . . . . . . . .

Acute Inflammation of the Kidney; Acute Nephritis-Chronic Nephritis-Suppurative NephritisDegenerations and Neoplasms of the Kidney-Parasites of the Kidney-Diabetes Insipidus-Diabetes Mellitus.

$$
\text { SECTION } \mathrm{X} \text {. }
$$

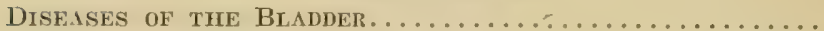

Cystitis-Spasm of the Sphincter Vesicæ-Paralysis of the Sphincter-Lithiasis; Stone in the Bladder -Retroflexion-Tumors-Prolapse-Eversion.

\section{SECTION XI.}

Drseases of the Prostate, Urethra and Prepuce.......

Urethral Calculi-Urethritis; Catarrhal Inflammation of the Mucosa of the Urethra-Prostatitis-Hypertrophy of the Prostate-Neoplasm; Tumor of the Prostate-Phimosis-Paraphimosis-Tumors of the Glans, Prepuce and Vagina.

\section{SECTION XII.}

Diseases of the Testicles and Scrotunt............ 103

Orchitis-Abcess of the Testicle-New Growths.

\section{SEC'IION XIII.}

Diseases of the Heart and Blood Vesseis........... 105

Pericarditis-Acute Endocarditis-Chronic Endocarditis and Valvular Defects-Hypertrophy and Dilatation of the Heart-Rupture of the Heart.

\section{SECTION XIV.}

Diseasts of tire Bloon ann Blond Vessels........... 111

Aneurysms-Hematozoa-Anemia of the BrainCerebral Hemorrhage.

\section{SECTION XV.}

Diseases of the Nemwous Systex...............

Encephalitis-Cerebrospinal Meningitis-Inflammation of the Spinal Cord and Membrane; Myelitis and Meningitis Spinalis-Apoplexy-Epilepsy-Chorea. 
SECTION XVI.

Diskases of THE Eye..................... 122

Conjunctivitis-Keratitis-Abscess of the CorneaOpacities of the Cornea-Congenital Opacities-Iritis -Cataract-Diseases of the Retina-Glaucoma.

SECTION XVII.

Diseastes of TIIE E.IR...................... 131

Ulceration of the Ear Flap-Catarrh of the Auditory Canal (Canker)-Deafness.

SECTION XVIII.

DISE.ISES of THE SKIX.................. 135

Sarcoptic Mange-Follicular Mange-Ringworm and Favus-Parasitic Dermatitis-Non-Parasitic Diseases of the Skin-Erythema-Eczema-UrticariaAlopecia-Acne.

SECTION XIX.

Infectious Coxstitutiox.l Diselses............ 148

Distemper-Rabies-Furious Rabies-Dumb Rabies -Tetanus-Anthrax-Tuberculosis-Granuloma.

SECTION XX.

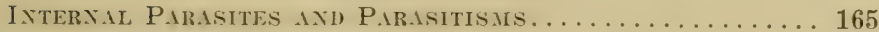

Spiroptera Sanguinolenta - Ascaris MarginataOxyuris Vermicularis-Ankylostomum Trigonocephalum-Tricocephalus Depressiusculus-Tænia; Tapeworms.

SECTION XXI.

Poisoxs axd Porsoxixg....................... 17

Pathology of Poisoning-Arsenic-PhosphorusStrychnin-Hydrocyanic Acid and Potassium Cyanid -Carbolic Acid (Phenol)-Iodoform.

\section{PART II}

Surgtcal Operations,

SECTION I.

Premimisaries

Anesthesia - General Anesthesia - Preparatory Treatment of the Operative Area-Preparation of Instruments-Preparation of the Operator's Hands.

SECTION II.

Surgiry of the Head and Neck................ 188

Hematoma of the Ear Flap-Plastic Operation for Split Ears-Harelip and Cleft Palate-Esophagotomy -Tracheotomy. 


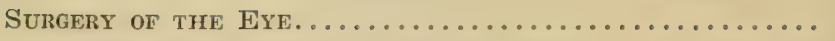

Injuries; Foreign Bodies-Warts and Foreign Bodies - Entropion - Ectropion - Pterygium - Displaced Orbital Gland-Enucleation of the Eyeball.

SECTION IV.

Surgery of the Thor 4 x $\Lambda$ nd Abdomen.............. 20 203

Paracentesis Thoracic-Paracentesis AbdominisParacentesis Vesicæ - Laparotomy - Gastrotomy Enterotomy-Enterectomy and Anastomosis of the Intestine-Ovariotomy and Ovariohysterectomy- $\mathrm{Hy}$ sterotomy-Cesarian Section-Suprapubic Lithotomy.

SECTION V.

OPERATIONS For Herixt...................... 275

Umbilical Hernia - Ventral Hernia - Inguinal Hernia-Scrotal Hernia-Perineal Hernia.

SECTION VI.

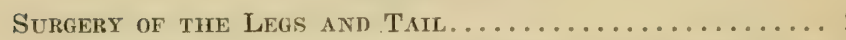

Sprains - Dislocations - Necrosis - Amputation of the Tail-Fractures-Amputation of - Claws-Interdigital Cysts-Tumors.

SECTION VII.

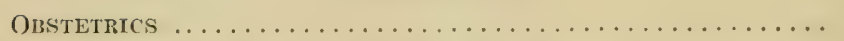

Dystocia-Maternal Dystocia-Fetal DystociaAccidents Liable to Occur During Parturition Exhaustion-Lacerations of the Vagina-Rupture of the Uterus-Sequelæ to Parturition-Vaginitis-Prolapse of the Vagina and Uterus-Metritis-Simple Metritis-Septic Metritis or Puerperal Fever-Mammitis-Eclampsia. 


\title{
CANINE MEDICINE AND SURGERY
}

\section{PART I \\ CANINE MEDICINE}

\author{
SECTION I
}

\section{GENERAL REMARKS}

7 HE examination of a sick dog must be made 1 carefully, so that a correct diagnosis of its condition may be reached, as on this the treatment and ultimate results largely depend. In this preliminary examination the animal must be handled gently but firmly, and so secured that it can do no injury to the veterinarian or to his assistants. In every case, unless the animal is known to be docile, its jaws should be secured either by a muzzle or by a clove hitch. "The non-observance of this precaution has led repeatedly to painful injuries, and in sereral instances in the writer's experience has made necessary a visit to the hospital for the Pasteur treatment.

The physical condition of the patient, and its mental attitude, should be noted, and its appetite, diet and the condition of the bowels and urinary apparatus should be inquired into. The temperature, the rate and character of the pulse and the respiration should also be noted. The nose of a well dog is moist and cool; in a sick dog it is usually hot and dry. The skin, normally, is loose and supple, and the hair bright and lustrous; in disease, 
on the other hand, the skin becomes dry, often hot to the touch, and it may be the site of eruptions, and in some places be devoid of hair. In wasting disease, skin ailnents and digestive derangements the hair feels harsh and dry, is staring in appearance, and is often brittle or easily pulled from the follicles. The condition and color of the visible mucous membranes are often valuable guides to diagnosis, and should always be examined, while the character and amount of any discharge from the eyes and natural openings of the body must on no account be overlooked.

Having made this general examination of the patient, a more searching and specialized one is next carried out. The information obtained by the general examination will determine what organ or organs it is necessary to examine more closely.

If the lungs, bronchial tubes or pleuræ are suspected, the special examination consists of auscultation and percussion to determine what abnormalities, if any, are present. The abdomen should be palpated, auscultated to determine the absence or presence of peristalsis, and moderate pressure applied over the abdominal walls to discover any unusual tenderness or tenseness of these walls. The eyes should be carefully scrutinized, and the action of the pupils noted, to learn whether the dilation is equal or unequal.

The writer cannot urge too strongly that throughout the examination all gentleness and consideration be shown the patient, both for humane and ethical reasons, and because of the fact that success in dog practice depends in no mean degree upon one's ability to "get along" with the mistress of the dog and win the confidence of the pet, both of which are impossible if the $\mathrm{dog}$ is handled roughly during the examination. 


\section{THE ADMINISTRATION OF MEDICINES}

Medicine may be administered as to other animals. by the mouth, hypudermically, intravenously or by the rectum. The first two methods, save in exceptional-cases, are those most frequently practiced.

\section{The Oral Method}

Medicine in solution, in emulsion and in pill or tablet form may be given orally, but the two latter are by far the easiest to administer. To administer a pill or tablet the animal is either held by an assistant or steadied against the operator's legs; the left hand is placed over the upper jaw and the cheeks pressed against the teeth, while the under jaw is gently depressed by the first finger of the right hand. As the mouth opens, pressure is maintained by the fingers of the left hand, thereby forcing the cheeks between the molars and effectually preventing the mouth closing. The pill or tablet is then placel well back on the animal's tongue and the mouth allowed to close.

In cases where it is desirable to administer the medicament in liquid form the dose should be regulated so as to be of as small a quantity as possible, except, of course, where irritant drugs are used, in which case they must be properly diluted to avoid irritation of the buccal mucous membrane. The medicine being already placed in a teaspoon, syring: or other convenient receptacle, the animal's mouth is closed, either by an assistant holding the jaws closely together or by tying them together with a tape. The index finger of the left hand is then introduced at the corner of the lips and the checks pulled ontwards to form a pocket, into which the medicine should be poured slowly until the animal has taken the required dose. 


\section{The Hypodermic Method}

This is one of the most effectual methods of administration where quick absorption, with consequently quick results, is desired. The requirements are non-irritating and easily soluble drugs, small-caliber needles and a twenty-minim syringe, preferably all metal, because such are easily sterilized. The site of injection is of no importance, provided the skin be loose and thin, and for this reason the region of the scapula is generally selected. The site of injection should be painted with tincture of iodin to disinfect it; the needle and syringe should be sterilized by boiling, and the drug completely dissolved in distilled water. Unless all of these precautions are taken, an abscess may result at the site of the puncture. The syringe being filled, the skin is pinched up by the index finger and thumb of the left hand, the needle pushed quickly through the skin into the subcutaneous tissues, by the right hand, and the syringe is then attached and its contents evacuated slowly.

\section{The Intravenous Method}

This method is not used extensively in dog practice, but is coming more into vogue than formerly. The skin over the vein selected for the injection, usually the auricular vein, must be most carefully disinfected before the needle is inserted. The needle and the syringe must also have been sterilized, distilled water should be used for the solvent, and the drug must be completely dissolved to avoid embolism with its attendant dangers. The temperature of the solution should be raised to the temperature of the animal, and great care exercised in introducing the needle into the vein. All air must be expelled 
from the syringe before injecting, and the injection made slowly.

\section{The Rectal Method}

Medicine is administered by the rectum chiefly in the form of suppositories for the treatment of some local conditions, such as hemorrhoids, althought both ether and chloral may be given by this method to induce anesthesia. The rectum is first emptied by an enema of warm, soapy water, then the suppository is introduced manually or the liquid injected by means of a syringe. Rectal injections to remove feces, to lower temperature and for other purposes may also be considered as medication by the rectum. 


\section{DISEASES OF THE UPPER RESPIRATORY PASSAGES}

\section{Simple Nasal Catarrh, Rhinitis, Coryza, Cold in the Head}

THIS condition, as the name implies, consists of 1 an inflammation of the nasal mucous (schneiderian) membrane. However, it sometimes extends to the mucous membrane of the sinuses of the head.

Cause.-Cold, infection and the inhalation of irritant gases and other substances, for example, chemical fumes, smoke, dust or pollen are frequent causes of this condition.

Symptoms.-The first symptom noticed is sneezing and pruritus of the nose, the animal trying to alleviate the itching by pawing at the nose or trying to rub it against something. The nose is hot and dry and the nasal mucous membrane hyperemic. In from twelve to twenty-four hours exudation from the mucous membrane takes place and a thin and watery discharge is established. This later becomes thicker and more tenacious, taking on a purulent character. This discharge must be differentiated from that of canine distemper, which is more sticky and of a greenish-yellow color, besides being nearly always of an offensive odor and often streaked with blood. The duration of an attack of nasal catarrl is usually. about one week.

Treatment.-Nasal catarrh should always receive treatment, since it opens the gates, as it were, to other infectious agents; and, furthermore, the 
catarrhal conditions may spread farther down the respiratory tract.

A mild laxative, such as olive oil or cascara extract, should be given, and the nose kept clear of discharge. Inhalation of medicated vapor gives great relief and soothes the inflamed mucous mem. brane. A few drops of oil of eucalyptus, creolin or Friar's balsam (compound tincture of benzoin), in a kettle of boiling water, is all the apparatus required for steaming a dog. Small doses of quinin bromid, given three or four times daily, are usually all the medicine required. Attention nust be pair to the surroundings, the patient being made comfortable in a warm, dry and properly ventilated kennel. The diet should consist chiefly of milk an! easily digested solid foods.

\section{Epistaxis}

Bleeding from the nose is usually the result of violence, such as a kick or blow; it may be slight or very profuse, according to the extent of the injury. It is also seen as a symptom in disease of the turbinated bones, in neoplasms of the nasal or other sinuses of the head, and also in some parasitic affections, such as from the Pentastoma taenioides and in hookworm discase (Uncinaria trigonocephala infestation).

Treatment.-The animal must be kept quiet, and cold, either in the form of cold water or ice packs, should be applied over the nasal sinuses. Spraying the nostrils and nasal mucous membrane with a 1-1000 solution of adrenalin chlorid will usually control the hemorrhage. However, in case the hemorrhage becomes alarming and fails to respond to the foregoing treatment, a full physiologic dose 
of atropin sulphate should be injected hypodermically.

\section{Laryngitis}

The most common cause of laryngitis is cold. The usual way the animal becomes affected is by lying near a hot stove or a fire until he is too warm, and then lying by a door where a cold draught strikes the neck. Constant barking, a habit of dogs when away from home, is another prolific cause. External injuries to the larynx, caused by the dog straining at the leash or the jerk-line being applied too vigorously by the dog trainer while training animals to the gun, are likewise considered among causative factors.

Laryngitis may also appear as a complication in distemper or in bronchitis.

Symptoms.-A cough is the first symptom of laryngitis. This may be quite violent, and at first is hard, harsh and dry. When exudation takes place the cough becomes moister and softer. Auscultation over the larynx reveals wheezing or rattling sounds; manipulation of the larynx is painful, producing an attack of coughing. The cough is also easily excited and aggravated by running, eating, drinking and by the administration of drugs. In severe cases, where the mucous membrane is much swollen, there is a well-marked difficulty in respiration; the temperature runs high, to 104 or 105 degrees Fahrenheit, and the appetite is impaired.

The average duration of an uncomplicated attack of laryngitis is about four days, when, if not relieved, it may run on into the chronic form, or sudden death may occur from edema of the glottis.

Treatment.-The animal must be immediately placed in warm and comfortable quarters, and an abundance of cold water provided and kept con- 
stantly before him. According to the situation and circumstances, antiphlogistin (cataplasma kaolini U. S. P.), hot applications constantly renewed, a Priessnitz compress, ${ }^{*}$ or the ammoniacal liniment (B. P.), must be applied over the region of the larynx.

The throat should be sprayed three or four times daily with an antiseptic solution, such as equal parts of listerine and water, or one dram tincture of iodin in one pint of normal saline solution. To control the cough administer codein sulphate in doses of from one-fourth grain to two grains, according to size and weight of dog. Glycoheroin (Smith) in teaspoonful doses is useful, as are also some of the preparations of heroin and codein now on the market. Where edema of the glottis threatens, guaiacol should be given and pushed to effect; tracheotomy should not be delayed if there are symptoms of impending suffocation. The following prescription has been used with success:

R Guaiacolis.......................... i

Glycerini...................... ii

Spts. vini rect................... iv

M. Sig. Give half a teaspoonful in four teaspoonfuls of water three times daily.

\section{Chronic Laryngitis}

This condition usually results from an acute attack or from some other grave respiratory disorder. It may also be caused by new growths, or it may be tuberculous. Except as a sequel to the acute form, the approach of chronic laryngitis is gradual and unaccompanied by constitutional disturbances other than those produced by the conditions of which it is a complication.

*For a description of the Priessnitz compress or bandage, see the footnote on page 48 . 
Symptoms.-The manifestations of chronic laryngitis are the same as of the acute form, but are much modified in intensity. The cough is not so easily excited, but is hard and dry, and is usually worse at night, being often of a spasmodic nature. On manipulation, the larynx is not so tender as in the acute form of the disease, though coughing can be produced by pressure upon it. Exercise and excitement likewise produce attacks of coughing, as does also exposure to cold winds or draughts.

Treatment.-This, as a rule, is not satisfactory, but should consist of sedatives, to allay the cough, and stimulant inhalations, such as steam medicated with turpentine or oil of eucalyptus. Medicinally, guaiacol in oil may be given, and the patient should be placed on a good tonic course and liberal diet. A sharp counterirritant over the laryngeal region is also of benefit in some cases. 


\section{DISEASES OF THE BRONCHI AND LUNGS}

\section{Bronchitis}

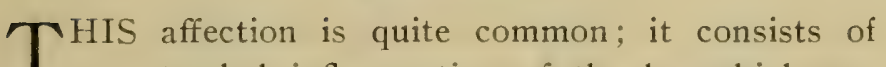
I a catarrhal inflammation of the bronchial mucous membrane, and occurs both in the acute and chronic form, which see.

\section{Acute Bronchitis}

Acute bronchitis may be due to any of the following causes: Infection; exposure to cold and wet draughts when warm from exercise, or from lying in front of a stove or fire; smoke, chemical fumes (inhalation bronchitis), medicines going "the wrong way," particles of food inspired when the animal is insensible, parasites, ether anesthesia, and as a complication of distemper.

The average duration of an attack of acute bronchitis is from two to three weeks.

Symptoms.-This disease is ushered in with shivering fits or rigors, and a rise of temperature to 103 or 105 degrees Fahrenheit. The animal is depressed and more or less indifferent to its surroundings; the bowels are costive; the urine highly colored and scanty. Soon a cough appears, at first dry and dull, but later, as the disease reaches the second stage, moist and loose. The cough can be quite easily excited by pressure on the thoracic walls behind the shoulder or by slight pressure on the trachea. At first only the large bronchi may be 
affected, but if the condition extends to the capillary air passages the above symptoms are much intensified. The respirations are quickened and labored, and the cheeks puff out at each expiration; the fever runs higher and the depression and general constitutional disturbance become more marked. The pulse at first is full and bounding, then becomes quicker and weaker, and in cases approaching a fatal termination is almost imperceptible. Percussion gives negative results. In the first stage, auscultation reveals that the respiratory sounds are harsh and dry (bronchus râle); in the second stage the mucous râle is well established, and the vascular murmur intensified. An attack lasts two to three weeks, and ends in recovery, chronic bronchitis, catarrhal pneumonia, stenosis, or asthma.

Treatment.-In smooth-haired dogs the thorax should be covered with a coating of cataplasma kaolini U. S. P. (or antiphlogistin, thermofuge, or similar remedy), applied hot, and covered with a thick covering of cotton wool, kept in place either by a bandage or by a pneumonia jacket, which can easily be made from factory cotton quilted with cotton wool or batting. This application should be removed and reapplied every twenty-four hours, for two dressings. In animals covered thickly with hair, oil of Sinapis, one part, and olive oil, forty parts, well rubbed in, should be substituted for the cataplasm, and the jacket applied as before.

A purgative is always indicated in acute bronchitis, and may consist of one half to two grains of calomel, repeated as occasion requires, it being most important to stimulate elimination from the outset of the disease. Unless the disease is of the wellmarked sthenic type and the fever extreme, the coal-tar antipyretics, such as phenacetin and anti- 
pyrin, should be sedulously avoided, on accuunt of their depressing action on the heart. The fever may be kept within bounds and elimination obtained far better by febrifuges and diuretics. The following is a satisfactory combination:

I* Potassii acetatis.................... xrs

Liquor ammonii acetatis...........dr. i

Spiritus etheris nitrosi.............min. $\mathrm{xxx}$

Infusion buchu ................drs. iv

M. Give four drams without water twice daily.

The cough may be regulated by one-fourth-grain doses of codein sulphate every hour.

In the second stage an expectorant cough mixture should be given, and stimulants as needed, of which there is none better than 1-200 to 1-60 grain of strychnin. Either of the following prescriptions may be used with good results:

If Heroin hydrochloridi................... i

Syr. tolutani,

Syr. scillæ, aa....................drs. vi

Aquæ chloroformi...................ozs. vi

M. Give two to four drams in water three times daily.

I Vini ipecacuanhæ................min. xii

Liquor ammonii acetatis,

Syr. codeinæ...................dr. ss

Syr. scillæ, aa...............min. $x v$

Aquæ ad....................drs. iv

M. Give four drams three times daily.

Should the bronchial tubes become loaded with mucus of which they are not able to rid themselves, and the animal's breathing becomes greatly distressed because of its interference with the passage of air, an emetic, of which the most effective is one thirtieth to one-tenth of a grain of apomorphin hydrochlorid, will give great relief, often averting suffocation.

The after-treatment consists of a liberal diet and 
tonics, such as Blaud's pills, one pill three times it day, or a granule composed of

If Strychnin arsenate................ 1-128

Iron arsenate,

Quinin arsenate, aa.................. 1-64

Nuclein solution.................min. iv

M. Give one half to three, three times daily, or give ferri et quininæ arsenas, two to ten grains, three times daily.

\section{Chronic Bronchitis}

This is a sequel to cases of acute bronchitis which, owing to neglect or other reasons, merge into the chronic. It runs a varied course of from a few weeks to several months.

Symptoms.-Continual coughing and expectoration is a symptom of chronic bronchitis. The cough is easily started by exertion or excitement, and the expectorated matter is tenacious and often of an offensive odor. The pulse and temperature as a rule are disturbed but little, although in some cases the latter may be slightly elevated. The animal's appetite is variable, and it generally loses flesh and becomes more or less emaciated.

Treatment.-The treatment consists in controlling the cough and the exhibition of expectorants, steaming with stimulating medicated inhalations, and the administration of tonics.

In this condition "Guaialyptol," a combination of guaiacol, eucalyptus, camphor, and phenic acid in oil has a markedly beneficial effect upon the mucous membrane and disinfects the entire respiratory tract. Cod-liver oil is also a most valuable reconstructive and should be given a trial. A liberal diet must be prescribed, and, as in all diseases of the organs of respiration, the importance of proper hygienic conditions must not be overlooked. 


\section{Pneumonia}

Like bronchitis, inflammation of the substance of the lungs is due to infection (pneumoccoccus, or Diplococcus pneumonix, and various Streptococci and Micrococci). It may also be caused by the spread of inflammation from other parts, as from the bronchial tubes in bronchitis or from the pleura in pleurisy. Again, it may be developed by the direct action of mechanical or other irritation, such as the inhalation of irritant gases or dust, or by vomition; or it may spring from the presence of emboli in the branches of the pulmonary artery or $o_{i}$ tubercles or clots in the lung tissue; it may also occur as a complication of heart disease, kidney disease, rheumatism, debilitating diseases, distemper, tuberculosis, or echinococcosis.

Cold and wet, unhealthful surroundings, and a lowered vitality are undoubtedly predisposing causes. Clinically, in the dog we have to deal with two forms of pneumonia, namely, lobar pneumonia, which is of rare occurrence, and lobular pneumonia, which is common. The words "diffuse" and "circumscribed," however, might be used to better advantage, the type of the former variety being furnished by the idiopathic affection; that of the latter by the condition which is secondary to diseases of the air passages. The two varieties, however, merge into one another.

\section{Lobar (Croupous) Pneumonia}

This type begins with hyperemia of the small vessels which are distributed in the walls of the air cells and bronchial passages, a swelling and tendency to proliferation of the epithelial cells of these parts, and an exudation of serum and cellular elements of the blood. The air vesicles and passages 
communicating with them gradually become filled and finally distended with this exudate, the air in them is expelled or absorbed by degrees, and the affected lung tissue becomes solid and heary. If the parts be now examined microscopically, the dilated blood vessels will be found crowded with their corpuscular contents, and the alveoli full of cells, all blended together into a common mass either by an amorphous, glutinous cement, or by a delicate, fibrillated network. With the progress of the disease the contents of the air vesicles liquefy and acquire a purulent character. This liquefied exudate is removed partly by expectoration and partly by gradual absorption. Occasionally, however, this breaking up of the exudate is followed by a breaking down of the lung substance, abscess formation, and gangrene.

The progress of a case of pneumonia through its various phases is quite gradual, but there are at least three stages that are more or less well marked. The first of these is the stage of engorgement (congestion, hyperemia), the second that of red hepatization, the third that of gray hepatization. In the first stage the lung still contains air, though in diminished quantity; it is deeply congested, exudes more moisture than is natural, is increased in weight, and is more friable than normal lung tissue. This condition of the lung tissue is difficult to distinguish from hypostatic congestion, for which it must not be mistaken if found on autopsy. In the second stage the lung is consolidated; the cells have lost their air and the cavities are filled with adherent masses of cells; it is distended to its full size, and its constituent lobules are distinctly mapped out upon the surface. In this stage the lung will sink in water 
and when sectioned it appears fairly dry and slightly granular, presenting a marbled aspect which is due to the intermixture of nearly colorless inflammatory exulate and areas of congestion. The third stage differs from the second mainly in the assumption by the affected lung tissue of a fairly uniform, opaque, grayish, yellowish, or greenish tinge, in its largely increased friability, and in the ready exudation from the cut surface of a thick, turbid, purulent fluid; in some cases this exudate is comparatively scanty; in others it is so abundant that the lung is like a sponge saturated with pus. Since pneumonia tends to spread, it naturally follows that different portions of the affected lung often present well-marked differences of condition, and that we occasionally find all the recognized stages of pneumonia present at the same time in the same case.

Inflammation may involve the lung to a varied extent; thus it may be limited to a section no larger than a walnut or it may include the whole lobe or even a whole lung; and further, it may affect both lungs. The right lung is affected more frequently than the left, and the lower lobe more often than the upper.

Symptoms.-Although of somewhat rare occurrence, the symptoms of lobar pneumonia should be known by the veterinarian. The disease is ushered in with a day or two of lassitude and dullness, anorexia, and restlessness. The invasion of the disease is generally marked by a rigor or a succession of rigors, and the temperature rises to 104 or 106 degrees Fahrenheit: the respirations are increased, in some cases up to sixty a minute. The breathing is shallow and labored and accompanied by more or less dyspnea; the cheeks are puffed out 
at each expiration. There is a short, dry, painful cough. The pulse is full and bounding, running at the rate of 150 to 190 a minute during the hyperemic stage, but becoming softer and weaker but still fast in the second and third stages. In cases approaching a favorable termination the pulse sradually regains its normal tone and rate, but in those cases that do not recover the pulse becomes weaker and weaker, until it is barely perceptible.

While pneumonia is in progress the systemic veins are apt to become overloaded and the mucous membranes may assume a cyanotic tint, although usually they are congested and of a reddish color. Thirst is always increased, but loss of appetite is invariable. The condition of the bowels varies, and though the patient is usually constipated, sometimes there is more or less diarrhea. The urine is scanty and dark colored.

In the first stage of lobar or croupous pneumonia the most observable auscultatory phenomenon is minute crepitation, which may be audible during the whole of inspiration, sometimes during expiration as well, and not infrequently at the end of a deep inspiration, such as that which precedes a cough. In association with this there may be no change or percussion or there may be high-pitched resonance. The second stage is marked by the supervention over the consolidated portion of the lung of cessation of the fine crepitation and the development in its place of well-marked tubular breathing; when, however, the bronchial tubes leading to these consolidated areas are completely obstructed there is total absence of respiratory sounds.

When pleurisy is coexistent with pneumonia, as is often the case, the friction sound indicative of 
that condition will also be heard. At a later stage. when the lung tissue is breaking down or resolution is taking place, tubular breathing gives way to a sort of coarse crepitation to which the name crepitatio redux has been given. This gradually passes into the ordinary bronchitic râles.

The sputum expectorated is of little diagnostic value in the dog, as it is almost invariably swallowed immediately it is coughed up. It is, however, at first transparent, very viscid, and tinged with blood, later it loses its sanguineous tint and becomes opaque and greenish, acquiring, in fact, a mucopurulent character, and then gradually diminishes in quantity. In some cases, instead of undergoing these changes, which may be regarded as the normal changes, the sputum acquires a deep purplish or reddish-brown tint, and at the same time a more watery consistency. This form of sputum has been likened to prune juice and is generally the sign not only of increased congestion and escape of blood but of the onset of the third stage. If attended by a disgusting fetor, it indicates the onset of pulmonary gangrene. In either case, it cannot but be regarded as an unfavorable symptom.

\section{Lobular (Catarrhal) Pneumonia}

This is the commonest pneumonia of the dog. In typical cases the lung is studded with pneumonic patches varying in size from that of a pea to that of a walnut, each involving one or more pulmonary lobules, circumscribed by the interlobular connective tissue and separated from one another by a network of still crepitant, and, it may be, of perfectly healthy lung tissue. The pneumonic patches may be in the hyperemic condition only. in which case they may not be recognized or may 
present the features of ordinary red or gray hepatization.

Further, by an extension of the disease, the neighboring diseased areas may coalesce and thus extensive tracts of lung tissue become involved. Full lobular pneumonia is always secondary to the blocking up of air passages, and especially those of capillary size; it may be excited either by the gradual extension of the inflammatory process from the tubes to the air vesicles, or by the entrance into the vesicles during inspiration of the inflammatory products of the tubes, which then act as irritants and carriers of infection.

Closely related to lobular pneumonia is the disseminated pneumonia due to obstruction of small branches of the pulmonary artery, either by embolism or thrombosis, or in the course of pyemia. In these cases, as in the other, the affected areas are of small size and limited by the margin of the lobules. But there is a greater variety of result, especially in pyemia; in which, while the affected areas sometimes present simple hyperemia, or red or gray hepatization, they not infrequently are the seat of hemorrhage, or undergo rapid suppuration or gangrene.

In all forms of pneumonia, even in such as are not of bronchitic origin, there is a tendency to the development sooner or later of bronchitis. But apart from this there is a marked disposition early in the course of pneumonia to the effusion into the tubes from the inflamed air cells of a transparent, very viscid fluid, uniformly stained with blood and containing cells; and in some rare cases this fluid, like that in the air cells whence it is derived, undergoes coagulation in the bronchial tubes, which thus 
become filled to a greater or less extent with casts consisting of coagulated fibrin and cells.

Fortunately, abscess formation is the exception rather than the rule in lobular pneumionia, the disease seldom going beyond the third stage.

Gangrene seldom occurs in idiopathic pneumonia, it being met with chiefly in those cases in which the pneumonia is secondary to or complicated with some other affection. It is characterized by the breaking down of the lung tissue into a fetid, dirty, greenish-yellow pulp, and by more or les.s greenish discoloration of the surrounding consolidated tissue. The gangrenous condition may involve either an extensive tract of lung tissue of several scattered areas or even a single small patch. The complications of pneumonia are pleurisy, bronchitis, icterus, and intestinal congestion. It is common to find on autopsy the right side of the heart filled with a fibrinous coagulum which is prolonged into the pulmonary artery (antemortem clot), while the left side is contracted and almost empty.

Symptoms.-The lobular type, the common pneumonia of the dog, attacks its victims insidiously during the progress of some other disease, such as distemper or bronchitis. Its onset is not usually marked by rigors or the violent circulatory disturbances noted in the lobar form. In patients suffering from bronchitis or distemper the supervention of this form of pneumonia may be suspected by an aggravation of the respiratory disturbance. The temperature mounts higher, the respirations are more labored, and the puffing out of the cheeks at each expiration more marked. Auscultatory and percussive phenomena are not so distinctive as in the idiopathic form, but apart from its insidious approach, longer course, and greater 
mortality, lobular pneumonia, with the exception noted, presents the same clinical symptoms as the idiopathic variety.

Treatment.-The treatment of puenmonia of either the lobar or lobular, type, resolves itself into the treatment of conditions found, and the upkeep of the vital forces of the patient until the disease runs its course.

The most modern method of treating pneumonia is by the pneumobacterin and antipneumococcic serum. The former combats the disease by raising the opsonic index of the patient, thus enabling the leukocytes to more effectually cope with the invading organisms (active immunity), and should be employed as soon as the diagnosis of pneumonia is positive. It may be used in doses corresponding to those used in human practice, namely twentyfive to fifty million dead bacteria injected hypodermically. It is not necessary to obtain the opsonic index, as variations in the temperature constitute a sufficient clinical guide. In cases responding to bacterin treatment the temperature will fall afte: the injection and then gradually rise again, when another injection should be given. The antiserum is used to neutralize the toxins already liberated by the organism and often produces great ameliora.tion of the symptoms (passive immunity).

One of the most important factors in treating this, or for that matter, any other infectious dis. ease, is to secure free elimination for both the toxins produced by the infecting agents, and also for those manufactured in the animal's own alimentary canal. In health these toxins are eliminated or neutralized, and their producers kept under control by the excretory organs and digestive juices. In disease, on the other hand, the functions 
of the excretory organs and, in fact, the whole mechanism of the body, is more or less interfered with and thrown out of its normal equilibrium. In other words, we have to deal not only with toxins produced by the invading organisms, but with toxins produced by the animal itself, that is, with an autointoxication.

Thus free elimination, both by the intestinal tract and the kidneys, must be maintained as the most essential part of the treatment. This may be accomplished by small doses of calomel, onefourth grain every two hours to effect, or until one to two grains have been taken, and by the diuretic mixture given for the treatment of bronchitis. Constipation must never be allowed to persist.

In sthenic cases, with a full, bounding pulse, the circulation must be equalized either by bleeding or, better still, by small, repeated doses of 1-1600 to 1-800 grain of aconitin every thirty minutes to effect, and then at intervals sufficient to keep the pulse within bounds.

The same application to the chest wall as given under bronchitis should be applied, or equal partis of guaiacol and camphorated oil rubbed in and the thorax covered with a padded jacket. Abundance of fresh air should be allowed. The cough may be controlled by the administration of codein, one. fourth grain as required, and expectoration facilitated by inhalations and expectorant mixtures. Guaiacol is an extremely useful drug in this condition, being an antipyretic, stimulant, and respiratory disinfectant.

In the second and third stages an ever-watchful eye must be kept on the heart, and stimulants given as conditions indicate. Strychnin, 1-200 to 1-60 grain, is the drug most useful here, and it may be advantageously combined with 1-50 grain of digi- 
talin. Throughout the iliness the diet must be light, nourishing, and easily digested. Milk, beef tea, and small quantities of finely chopped raw beef are appropriate articles of diet. In cases where the animal refuses to eat at all, extract of meat, made into pills, may be administered, or a teaspoonful each of brandy and beef tea may be given every two hours.

The after-treatment of pneumonia consists of liberal diet, moderate exercise, and a good, iron tonic.

In cases of pneumonia that take on a septic character due to secondary infection (pyogenic bacteria), all the usual symptoms are accentuated, there is the addition of great prostration, and the case only too often ends fatally. However, antistreptococcic serum in full and repeated doses has in many cases saved life, and should be resorted to as soon as the secondary infection is diagnosed. Subcutaneous injections of camphor are particularly useful in these cases and should be repeated as required. Thirty to sixty minims of spirit of camphor may be given, or it may be given as follows :

IF Camphoræ ....................... $x$

Etheris sulphurici.................. ss

Olei olivæ......................drs. ii

M. Inject fifteen to thirty minims, according to the circumstances.

Gangrene of the lung is often the sequel of the secondary invasion in pneumonia and is nearly always fatal, but the above treatment should be persisted in, in the hope of inducing a favorable termination. 


\section{S E C T I O N I V}

\section{DISEASES OF THE PLEURE}

\section{Pleurisy}

\section{(Inflammation of the Pleura; Pleuritis)}

The disease is found in two forms, primary and secondary pleuritis. The primary form is usually caused by exposure to cold, especially when the coat is wet, as in sporting dogs after swimming, or running through wet underbrush. Secondary pleurisy appears as a complication of other diseases, such as pneumonia, pericarditis, peritonitis (extending from the abdominal cavity through the diaphragm), fractured ribs, punctured wounds of the thorax, perforation of the esopliagus by foreign bodies, tuberculosis, or pyemia.

Morbid Anatomy.-The pleura is dull and tumefied and very hyperemic. Its surface is roughened by accumulations of fibrin and in appearance may be likened to the surfaces of two pieces of bread and butter that have been placed together and then separated. When there is no accumulation of flaid it is termed dry pleurisy, or pleuritis fibrinosa.

Usually, however, exudation takes place and an accumulation of a serous fluid in which fibrinous coagula float, is found between the pleural folds. This pleuritic fluid, closely resembling blood serum, contains cells, and, according to the number of red blood corpuscles it contains, is of a straw color or of a sanguineous color.

In secondary pleuritis, infection with pyogenic organisms may take place and then the fluid be- 
comes purulent, constituting the condition known as septic pleuritis or empyema. In primary pleuritis the condition as a rule affects only one side of the chest, while in secondary pleuritis it affects both sides, but this is no hard and fast rule.

The prognosis in primary pleuritis is generally favorable, though convalescence may be slow; that of the secondary variety depends entirely on the primary cause, or trauma.

Symptoms.-Pleurisy may or may not be ushered in by rigors, but there is always malaise and great stiffness in action, and disinclination to move or bend the body, the condition being very painful. The respiratory movements are painful and almost entirely abdominal, the animal, on account of the pain attending thoracic movement, trying to fix the chest walls as much as possible. The constitutional disturbance is great, thirst is greatly increased, anorexia generally complete; the bowels are costive; the urine is scanty, highly colored, and contains albumin; the pulse is fast and wiry, the temperature 104 to 105 degrees Fahrenheit; the mucous membranes are congested.

Until the effusion has taken place percussion yields negative results. Afterwards a dull sound is heard up to the level of the accumulated fluid.

Auscultation reveals the characteristic friction sounds of pleurisy, or later the fluid may be heard splashing during respiration. Since the accumulation of fluid in the pleural cavity compresses the lung, sometimes to complete collapse, dyspnea is always well marked when effusion has taken place. and when the effusion is great symptoms of asplyyia manifest themselves; the compression of the heart and great blood vessels also leads to their disturbed and impaired action. 
Where the effusion is great in amount the intercostal spaces may be seen to bulge outward and the whole side of the chest is thus altered in appearance. If empyema supervenes, the pulse becomes rapid and weak, and the temperature is intermittent but always high. Sometimes, however, the first indication of the empyemic condition is the expectoration of pus or a pointing in an intercostal space; as the evacuation of the pus in empyema must not be delayed, every endeavor should be made to diagnose it early. If the presence of pus is at all suspected, an exploratory puncture should be made.

Cough may or may not be present in pleurisy; when present it is always painful, dry and suppressed.

Treatment.-At the onset of the disease there is hardly any drug that will combat the pain and fever and equalize the circulation better than aconite or its alkaloid aconitin. Either may be administered in small doses every fifteen minutes for two hours, and then hourly, until the effusion takes place. The tincture of aconite in two minim doses, or 1-800 grain of aconitin, or a defervescent compound (aconitin, 1-800 grain; diditalin, 1-6t grain; veratrin, $1-128$ grain), is perhaps the most useful.

Aconitin, it must be remembered, is a powerful drug, and its frequent administration must be stopped as soon as either the desired effect is produced or the physiological action of the drug brought about, as manifested by the softening of the pulse, champing movements of the jaws, and general relaxation of the patient. When this has been attained the dose is given at longer intervals to maintain the effect. Counter-irritants should 
be applied to the thoracic walls, either in the form of cataplasma kaolini, or mustard or oleum sinapis, and a firm supporting bandage placed around the chest to immobilize the ribs. The relief from pain given by thus fixing the ribs is most marked.

When effusion has taken place, efforts must be made to hasten its reabsorption and elimination. This may be done by purgatives and diuretics. By their alterative and diuretic actions potassium iodid or ammonium iodid is of great service here. Codein in one-fourth grain doses, repeated as required, will control the cough which, if present, is always distressing. Where the effusion is so excessive as to cause great difficulty in breathing, with symptoms of asphyxia, paracentesis thoracis should not be delayed, but promptly performed. The main danger lies in delaying the operation after the necessity for it is recognized, the patient's vitality then not being sufficient for it to recuperate. The after-treatment consists in protecting the animal from exposure to cold and wet, liberal diet, and a line of alterative and tonic treatment.

\section{Hydrothorax}

\section{(Dropsy of the Thoracic Cavity)}

Hydrothorax, strictly speaking, is true dropsy of the chest, although the term has been loosely used in connection with the effusion found in pleurisy. Hydrothorax is a transudation, non-inflammatory in origin, and is the result of some other pathologic condition, such as cardiac or renal disease.

Symptoms.-A gradual filling up of the thoracic cavity with the transuded fluid, with corresponding difficulty in respiration, is the usual symptom of hydrothorax. Percussion reveals a distinct level of the fluid in the cavity, parallel to the horizon. 
The normal respiratory sounds may be heard above the level of the fluid, and indistinctly or not at all below the level. Splashing sounds are also in evidence. The other symptoms will depend upon the primary condition.

Treatment.-Treatment for this disease is usually unsatisfactory. . It must first of all be directed to the primary disease, and unless this can be diagnosed and corrected the treatment of the hydrothorax can be only palliative. It consists in the exhibition of diuretics and in resorting to paracentesis thoracis. This operation is harmless and may be repeatedly performed to relieve the dyspnea, but as a rule gives no permanent relief unless the primary cause is curable. On the other hand, by its repeated performance the life of an old favorite may often be prolonged for a considerable time.

\section{Pleurodynia}

Pleurodynia is essentially rheumatism of the muscular walls of the thorax. This exceedingly painful condition might possible be mistaken for pleurisy unless a careful examination is made. It is due undoubtedly to exposure to wet and cold, more especially in rheumatic subjects.

Symptoms. - The animal is dull and listless, and evinces pain on movement and upon being handled. The breathing is shallow and abdominal, the patient trying to use his respiratory muscles as little as possible. When compelled to move, the animal shows great stiffness and disinclination to bend the body. The pulse may be a little more rapid than normal, but has not the wiry characteristic of the pulse in pleurisy. The temperature, as a rule, is about normal, or a little above it. Auscultation and percussion reveal nothing. 
Treatment.-Counterirritants to the affected muscles, capsicum linament or mustard, and the application of the padded pneumonia jacket should be resorted to. The region on each side of the spine should be palpated, and spots tender to pressure should receive smart counterirritation. The patient should receive a saline laxative, such as one to four drams of magnesium sulphate, Its head being kept elevated for twenty minutes after its administration to prevent it being vomited, 'and acetyl salicylic acid in doses of five to fifteen grains. Asperin (acetyl salicylic acid) is far superior to the ordinary salicylates, because it is soluble only in an alkaline medium and it does not cause the gastric disturbances that other salicylates cause. Bryonin, 1-20 to 1-40 grain, every two to six hours, will usually give prompt relief.

In addition to the medical treatment, the patient must be kept in a warm, dry kennel and not exposed to cold or wet. The diet should be of a light and easily digested nature, such as milk, oatmeal porridge, or dog biscuit, while meat should be withheld until the attack is over. 


\section{SECTION V}

\section{DISEASES OF THE MOUTH, PHARYNX, AND ESOPHAGUS}

\section{Stomatitis}

STOMATITIS, or inflammation of the buccal $S$ mucous membrane, is the result of either chemical, thermal or mechanical irritants, and is often a complication or symptom of some other disease or condition. It is also common during teething, and where there are decayed teeth.

Symptoms.-The patient is observed to be slow and careful in eating, large pieces of food being discarded and smaller portions bolted without mastication. Salivation is a prominent symptom, the saliva hanging out at the corners of the mouth in glairy strings. Upon examination the mucous membrane of the mouth will be found to be red, swollen, and inflamed, the amount of inflammation depend.ing on the cause. In the case of corrosive poisons having been taken into the mouth the mucous membranes may be greatly eroded and hanging in shreds, the gums and tongue are also inflamed and tender, and ulcers sometimes appear on different parts of the mouth.

Treatment.-The dog's diet must consist of bland and non-irritating liquids or mashes, such as soups. milk, beef tea, bread and milk, and boiled rice; plenty of cold water should be supplied constantly.

The mouth should be kept clean by means of antiseptic and mild astringent washes, applied either with a camel's-hair brush or an atomizer; decayed 
and loose teeth must be removed and the tartar cleaned from the remaining teeth. In teething, the inflamed gums may be lanced.

Any of the following solutions constitutes a useful mouth wash for this condition:

1. Potassium permanganate, one-per-cent solution.

2. Peroxid of hydrogen, one to five parts water.

3. Borax, 130 grains; sodium bicarbonate, 130 grains; carbolic acid, twenty-five grains; glycerin, five one-half drams, and water to make twenty fluid ounces.

Ulcers may be touched with tincture of iodin or a strong solution of alum. The tender gums may be painted with a tincture of myrrh, or one part tannic acid to four parts glycerin.

\section{Stomatitis Ulcerosa, or Necrotic Stomatitis}

This condition is a necrotic inflammation of the mucous membrane of the mouth. It attacks the gums more frequently than other parts.

Cause.-This serious condition is generally seen in old, debilitated dogs and in weakly, anemic, and pampered animals of all ages. It is usually associated with decayed teeth, and is no doubt due to some microörganism, probably the Bacillus necrophorus, or possibly to various organisms. It is possible that the "Black Tongue" of the Southern states is a modified (intestinal), type of this same infection.

Symptoms.-The gums in the neighborhood of certain teeth become swollen, red, and painful to the touch. In a day or so there is marked increase of the swelling, and the affected gum is of a dark red, brown-red, or of a purplish hue, while the tissue affected is spongy, bleeds on the slightest pressure, and is seen to have receded from the teeth. Later, beginning with the swollen region next to 
the teeth, the affected part takes on a yellowish or greenish-yellow color. This necrotic tissue is of a pulpy, greasy consistency, and if removed, either by the sloughing process or by force, leaves a large ulcer about one sixteenth of an inch deep, with irregular, ragged edges.

The walls of the ulcer are hard and hyperemic and raised above the general surface of the gum. As the gangrenous process proceeds, abscesses form in the alveolar process, and the teeth become loose and fall out; in extreme cases, the jaw itself may become necrotic and portions of the bone exfoliate.

The morbid process extends from the gums to the lips and to the cheek, corners of the mouth, an 1 in rare cases to the tongue. There is always a profuse salivation, the discharge being ropy, glairy, blood-stained, and of an intolerably fetid odor. There is usually a rise of temperature to 103 degrees Fahrenheit, or even higher if septic absorption takes place, in which case the patient usually dies of septicemia.

The appetite is generally good, although mastication is both painful and difficult. In cases that run a benign course the necrosis is usually confined to the mucous membrane, and after the separation of the slough, the ulcers heal rapidly, often being entirely well in from eight to ten days. In the case of old, debilitated subjects the disease more often assumes a malignant type, the gangrenous and necrotic processes extend rapidly, and the animal dies from septic absorption.

Treatment.-All loose and decayed teeth must first be extracted and abscesses evacuated. The mouth must be frequently swabbed with a deodorant mouth wash, such as potassium permanganate in one-per-cent solution or hydrogen peroxid, and after- 
wards strong disinfectants applied. Creolin in twoper-cent solution, painted on with a camel's-hair brush, is most effectual. Lugol's solution* of iodin applied in the same manner often gives good results, and touching up the ulcers with caustics is also effective. Internally the patient should receive an occasional laxative and an iron tonic. Nuclein (eight minims hypodermically twice daily) should be given from the beginning of the treatment, and the animal's strength kept up by a liberal and easily digested diet.

\section{Glossitis}

Inflammation of the tongue is usually the result of injury by foreign bodies becoming embedded in or lacerating the substances of the tongue. Among the common causes of this condition may be mentioned spicules of bone that become lodged between the molar teeth, elastic bands placed around the tongue either by children in play or by some malicious person, irritant medicines, and caustics, new growths (carcinoma, sarcoma), etc.

Symptoms.-The animal shows difficulty in mastication and in lapping water. Salivation is present, the tongue is red, swollen, and tender, and the breath is often offensive.

Treatment.-A careful examination of the tongue must be made for foreign bodies, which, if present, must be removed. The teeth must also be examined for particles of bone wedged between them, and if any are found they must be removed. Wounds of the tongue heal with remarkable rapidity, and often the removal of the cause and the use of some mild antiseptic mouth wash complete the cure.

\footnotetext{
*Lugol's solution consists of: Iodin 5 parts; potassium iodid 10 parts; water q. $\mathrm{s}$. 100 parts.
} 


\section{Ranula}

Ranula is a large, fluctuating swelling which appears at the lateral or under surface of the tongue, usually near the frenum. It is painful, produces profuse salivation, and may attain such size as to prevent the complete closure of the mouth.

Cause.-This trouble is due to the blocking up or closure of the mucous gland and the consequent enlargement of the gland. An obstruction of Wharton's duct sometimes produces a similar condition. The contents of ranula -are of a thick, glairy, mucoid nature.

Treatment.-This should be surgical, the instruments required are a mouth speculum, a small scalpel, scissors, and tissue forceps. The instrument having been sterilized by boiling for twenty minutes, the mouth is fixed open with a mouth speculum and the tongue drawn into such a position as to place the ranula in the most suitable situation for removal. The sac is then punctured with the scalpel and the whole of the wall dissected out; unless this is thoroughly done, the ranula will recur. After-treatment consists in washing out the wound three or four times a day with an antiseptic and astringent mouth wash. Boracic acid, ten grains to the ounce, and chinosol, one-half grain to the ounce of water, make useful antiseptic washes.

\section{Epulis}

This name is given to a hard, smooth, periosteal tumor which appears on the gums at the edge of the alveoli. There may be only one tumor, or they may be numerous, and may be benign or malignant in character. Unless these tumors cause pain and undue inconvenience in eating it is better not to interfere with them. If an operation is decided upon, they may be removed with a scalpel or scis- 
sors and the hemorrhage controlled by styptics. Occasionally a bony attachment may be found, in which cases bone forceps will be necessary to complete their removal.

\section{Papilloma or Wart}

Papillomas are often multiple, and may cover nearly the entire surface or the mucous membrane of the lips.

Cause.-These tumors are of infectious origin.

Treatment.-In many instances papillomas disappear spontaneously without treatment, and the' animal is immune against a second infection. Where they are very numerous, local applications of a saturated tannic-acid solution should be applied and Fowler's solution given internally for at least a month before resorting to surgical means. If, however, there are only a few tumors present, or the above treatment is futile, they may be removed by ligature or with the thermo cautery. It is best not to excise them with the scalpel or scissors, as the blood may infect a new area.

\section{Tumors of the Mouth}

The new growths met with in the mouth and pharynx of the dog are mostly carcinoma, sarcoma, and adenoma. Usually they are found affecting the soft palate, the pharynx, and the vicinity of the tonsils, sometimes even invading the eustachian tubes. The cause of new growths is unknown.

Symptoms.- At first the patient shows some difficulty in swallowing, and the appetite may be capricous. Upon examination the growth may be discovered or, if deep seated, an wnhealthy looking ulcer with ragged edges may be seen. This may be mistaken for an injury caused by a bone or some other foreign body, but the involvement of the 
neighboring lymphatic glands and the non-response to treatment in time reveal its true character. The diagnosis may be absolutely confirmed if a small portion be excised and submitted to an expert pathologist for microscopic examination. In all cases there is rapid emaciation, and sooner or later death relieves the sufferer.

Treatment.-There is no successful treatment for this condition. Surgical interference appears to stimulate the malignant properties of these neoplasms, and the most humane course, once the diagnosis of malignancy is positive, is to end the animal's suffering by an overdose of chloroform.

\section{Harelip and Cleft Palate}

These congenital malformations may occur conjointly or singly, and are serious defects. More especially is this true of the latter, as it is impos: sible for the puppy to feed in the natural way and it has to be fed from a feeding bottle with a nipple large enough to reach to the back of the mouth.

A harelip may be double or single. A cleft palate may he wide or narrow, existing either in the anterior or posterior portion of the mouth, or it may extend the whole length.

Treatment.-The treatment is surgical, and consists of scarifying the edges of the lips or palate and uniting them by sutures. The details of this operation are given in Part II (see page 191).

\section{The Teeth}

The dog has forty-two teeth, formulated thus:

\begin{tabular}{|c|c|c|c|c|c|c|c|}
\hline & i. $3-3$ & c. $1-1$ & pm. 4-4 & $\mathrm{m}$ & $=42$ & & \\
\hline & $3-3$ & $1-1$ & $4-4$ & & & & \\
\hline & INC & OHS & CAxIxH & PRE & & Mo & \\
\hline JAW. & r. & 1. & r. $\quad 1$. & r. & 1. & r. & \\
\hline Upper & ....3 & 3 & 1 & 4 & 4 & 2 & 2 \\
\hline Lower & $\ldots .3$ & 3 & 1 & 4 & 4 & 3 & 3 \\
\hline
\end{tabular}


The first incisors and the canines, and the second, third, and fourth molars appear in the dog: at the end of five weeks. The permanent teeth begin to appear about the third or fourth month; the canine and middle incisors about the fourth month, and the remaining incisors at the end of five months, as well as the second, third, and fourth molars, the fifth molar about five months, the sixth about the sixth month, and the seventh about the end of the seventh month. Then the dog has a "full mouth" at the end of the seventh month.

During teething the gums become red and tender and the flow of saliva is increased. In some case 3 the appetite may be entirely suspended and convulsions may occur from reflex nervous irritation. In such cases the proper treatment is the administration of simple sedatives, such as potassium bromid (five grains three times daily), and scarification of the gums.

\section{Tartar}

Tartar is a calcareous deposit on the neck of the tooth at the border of the gums. It is deposited chiefly around the canine and molar teeth, and causes great irritation of the gums, sometimes even loosening the teeth and giving the breath an offensive odor. Tartar should be removed by scraping the tooth with a specially constructed instrument termed a "scaler," the mouth either being closed with a tape or kept open by means of mouth speculum. In powerful, violent patients a general anesthetic may be necessary to facilitate the operation of removal. The teeth should then be cleaned with a toothbrush and camphorated chalk or other suitable dentifrice. 


\section{Caries Dentum}

\section{(Caries of the Teeth)}

True caries of the teeth-that is, molecular destruction-is rare in the dog, but such cases have been observed. It begins on the upper surface and mainly in the cavity of the crown, appearing as a black spot. This spot, which is the decaying part of the tooth, gradually penetrates the tooth towari the pulp cavity, and finally exposes the nerve, toothache being the result.

In necrosis of the teeth there is usually an alveolar periostitis associated with it, the tooth becoming loose and yellow in color. In these cases alveolar abscesses may form and pyorrhea alveolaris, or dental fistula, remain. An animal affected with toothache becomes irritable, resents any manipulation of the mouth, has difficulty in mastication, and is salivated. The affected tooth is very tender; if struck with a sound or key causes the animal to cry.

Treatment.-In true caries, if treated before the nerve is exposed, the tooth may be saved by carefully drilling out the carity, removing all diseased tooth substance, and filling the cavity with dental cement or amalgam. If, however, the case has gone too far for filling, extraction is the only remedy. For this purpose the mouth is held open by a speculum or gag, the forceps applied as far on the tooth as possible, the tooth loosened by a few lateral twists and finally extracted by a strong pull in the direction of its roots. Immediately after the extraction the gums should be firmly compressed and the mouth washed out with an antiseptic lotion. 


\section{Empyema of the Superior Maxillary Sinus}

Necrosis of the fourth upper premolar often leads to empyema of the superior maxillary sinus, into which its roots penetrate, the pus discharging by a fistula just below the eye. This fistula may heal for a time under an antiseptic treatment, but sooner or later the discharge is reëstablished. By the use of a probe and tapping the affected tooth the significance of the fistula is recognized.

Treatment.-The fourth premolar niust be extracted, the probe pushed down the fistula into the mouth, the sinus syringed out with an antiseptic, and free drainage maintained. The syringing is continued until healing takes place.

\section{Pharyngitis, or Sore Throat}

Cause.-Pharyngitis, or inflammation of the mucous membrane of the pharynx, may be caused by the extension or spread of inflammation from contiguous organs, by irritant medicines and chemicals. foreign bodies, or by injuries caused by unskillful attempts at removing obstructions lodged in the throat or in passing the probang.

Symptoms.-Pharyngitis is manifested by a somewhat stiff carriage of the head; difficulty in swallowing solids; sometimes by coughing, especially if laryngitis exists also, and by a congested appearance of the affected mucous membrane.

Treatment.-The throat must be examined for foreign bodies, and if any are found they must be removed: this often presents considerable difficulty in the case of needles or pins, and great care must be exercised to avoid breaking them. The throat should then be sprayed either with iodin solution, 
hydrogen peroxid, or Dobell's solution of the following formula:

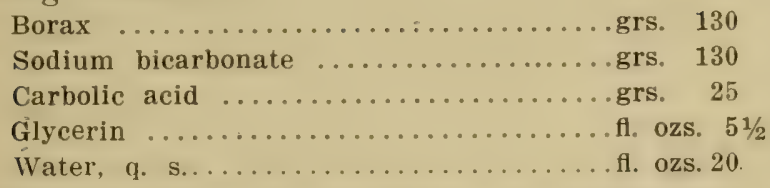

The diet must be restricted to liquids and soft foods to avoid irritation of the inflamed mucous membrane.

\section{Parotitis}

\section{(Inflammation of the Parotid Gland-Mumps)}

Cause.-There are four varieties of parotitis (or parotiditis), the causes of which differ. They may be classed as follows:

Contagious parotitis, the specific organism of which has not yet been determined; traumatic parotitis, produced by injuries; metastatic parotitis, due to a metastasis of pyogenic organisms during attacks of distemper, pyemia, or septicemia; and parotitis caused by the extension of a neighboring inflammatory focus, as in pharyngitis, mastoiditis, or similar diseases.

Symptoms.-In the contagious form the whole gland, or both glands, are swollen and tender, but there is no tendency to suppuration. In the other varieties the swelling is more circumscribed and suppuration usually takes places. The swollen glands are hot and tender and the act of swallowing is difficult and painful. Constitutional symptom:; are fever, anorexia, and lassitude, while in suppurative cases fluctuation is detected and the fever is usually high.

Treatment.-The patient should receive a mild laxative-olive oil or cascara-and the affecteil glands, after being freely fomented with hot water. 
should receive an inunction of warm camphorated oil or have calaplasma kaolini applied.

Moist heat in the form of Priessnitz compresses* often gives great relief by lessening the tension of the parts, and in cases where abscess formation is taking place it hastens the formation of the pus. Abscesses should be evacuated, drainage established, and the wound treated antiseptically: The diet must be of a liquid nature, such as beef tea, milk, and soft mushes.

\section{Foreign Bodies in the Esophagus, and Choking}

Choking is caused by the lodgment in the throat or esophagus of some foreign body, and is usually the result of greediness in feeding. The most common foreign bodies are bones, gristle, needles, and pieces of wood.

Symptoms.-The patient is greatly distressed, efforts at deglutition are greatly increased, and there is a return of food and drink through the nostrils.

Treatment.-Sometimes the offending article may be forced back into the mouth by manipulation of the exterior parts, and in some cases extraction with throat forceps is easy; but in cases where neither of these methods is successful the probang must be

*Describing the Priessnitz bandage or compress and its use, Glass says:

"The object of the compress or bandage is to maintain a con" tinual heat, either dry or moist, to certain parts of the animal's body. We first apply to the part affected a piece of absorbent cotton, thick wool, or dry felt; or if moisture is required, it is soaked in warm water or a medicated solution and wrung out to remove the excess of fluid: this is then held in position by a covering, of some light material-a wide bandage of cheese-cloth is the best-next a layer of oiled silk or rubber cloth (the object of this is to retain the heat and in case of a wet compress, the moisture), and finally over this is placed a compress or bandage of flannel. This last is to prevent loss of heat hy racliation. Sometimes the inner layer of cheese-cloth is omitted, or it may be put on the outside of all.

"The above procedure may seem to the liuried practitioner a rather long, and unnecessary method, but after one has tried it and found the great advantages it has in the retention of heat. especlally in diseases of lungs, in hastening the maturing of an abscess, or in the lessening of a tumefaction by the constant and direct application of heat and moisture, he will realize its benefits." 
passed and the offending borly cautiously pushed on to the stomach. In the case of fish bones or needles the operator may be able to entangle them in the horse-hair portion of the probang and extract them.

Great caution must be exercised not to use undue force or the esophagus may be ruptured, with serious, if not fatal, results. Fuller directions for passing the probang and for esophagotomy are given in Part II, Section II (see page 192). 


\section{DISEASES OF THE STOMACH AND INTESTINES}

$\mathrm{I}^{\mathrm{N}}$ the dog, impaction of the stomach and gas1 tritis constitute about sixty per cent of all diseases of digestion. This is due mainly to the want of proper attention to the animal's diet and to the practice of feeding only once a day, whereby the animal seeks to satisfy its hunger in a voracious manner.

\section{Impaction of the Stomach}

Cause-_Overfeeding and improper food are the principal causes of impaction.

Symptoms. - Vomiting is an evidence of this trouble, and if it takes place may empty the stomach and so relieve the condition, but in the majority of cases vomiting is not violent enough to give much relief, and the patient will retch, slaver, and eructate gas. Pressure over the epigastric region produces pain, the respirations are accelerated, and the patient is greatly depressed. There are colicky symptoms, with great uneasiness and a frequent desire to change positions. The animal groans or whines, and may be irritable and "snappy."

Treatment.-Empty the stomach by a hypodermic injection of 1-20 to 1-10 grain of apomorphin. Enormous masses of undigested or partially digested food are usually vomited, and relief is almost instantaneous. The stomach may then be washed out by means of the stomach pump, or eight to ten ounces of water may be administered and the 
dose of apomorphin repeated to more thoroughly clean the stomach. A gastric sedative, such as from five to twenty grains of bismuth subnitrate, may then be administered and the animal's diet restricted for a week or longer to small quantities of easily digested food:

\section{Gastritis}

This ailment, due to inflammation of the mucout: membrane of the stomach, occurs both in the acute and the chronic forms.

\section{Acute Gastritis}

Cause.-Acute gastritis is caused by irritating foods or medicines, such as decomposing meat or arsenic, corrosive sublimate, or phosphorus, as well as by foreign bodies. Acute gastritis also occurs as a symptom of distemper.

Symptoms.-There is romiting, with little or no appetite, and increased thirst, great quantities of water sometimes being taken, only to be immediately vomited. There is more or less constipation. The temperature is raised and the pulse accelerated. Pressure on the stomach causes pain. The patient is greatly depressed, lying down a great deal and assuming an almost diagnostic attitude, by trying to rest its abdomen on the floor.

Treatment.-If an irritant-poison, decayed meat, or a foreign body is suspected to be the cause, an emetic should be administered to clean out the stomach. The vomiting may be controlled with from five to twenty grains of bismuth subnitrate, combined with three grains of cerium oxalate or five grains of chloretone. The constipation may be overcome "by laxatives and enemas. If the pain 
indicates it, morphin in one-fourth-grain doses may be given subcutaneously, but chloral hydrate must never be used on account of its irritating effect on mucous membranes. In cases where there is, excessive tenderness on pressure, warm fomentations or turpentine stupes, or even the application of mustard over the epigastric region, often afford great relief. The animal must be kept on a low diet for some days; equal parts of milk and lime water may be given, and beef tea or finely minced, raw beef in small quantities. The after-treatment consists of a stomachic, such as a combination of quassia and strychnin, or tincture of rhei and tincture of nux vomica.

\section{Chronic Gastritis}

This disease is often the sequel to repeated attacks of the acute form; it may appear also as a secondary complication in other diseases. Generally, however, it is the result of continued improper diet, associated with à debilitated state of the system.

Symptoms.-Evidences of chronic gastritis are somewhat similar to those of acute gastritis, with the difference that the appetite is not entirely lost but is extremely capricious. Vomiting comes on only after eating, and there is no fever, and seldom is there evidence of pain on pressure over the epigastrium. The patient gradually becomes emaciated.

Treatment.-Constipation or diarrhea must be corrected at the outset and the diet previously indicated for acute gastritis prescribed. Internally a combination of two grains of pepsin, two and onehalf grains of bismuth subnitrate, and 1-100 grain of strychnin may be given immediately after meals 
three times daily, followed later with an alterative and tonic mixture such as:

I?

Tr. nucis vomice................drs. 4

Liqr. acidi arsenosi...............drs. 1

Tr. gentianæ ................. oz. 1

Syr. aurantii ................ oz. 1

Aquæ, q. s.......................... 4

M. Give two drams three times daily.

Or

13

Ac. nitrohydrochlorici ...........drs. 2

Tr. gentianæ comp................ oz. 1

Syr. aurantii .................. oz. 1

Aquæ, q. s........................... 4

ii. Give one dram three times daily, after meals.

The diet must be carefully regulated to avoid a recurrence of the trouble, the animal being fed small quantities at a time and several times daily. Proper exercise, grooming with a hound glove, and sanitary surroundings are valuable aids to treatment that are frequently overlooked.

\section{Foreign Bodies in the Stomach}

The variety of substances which may be swallowed either intentionally or accidentally while at play is so extensive as to prohibit a coniplete list; but sticks, stones, pins, needles, bones, coins, rubber balls, and corks are among those generally met with.

Symptoms.-In many instances the patient shows no ill effects unless the foreign body either perforates the stomach or, passing on, blocks the intestines. Silver coins may remain in the stomach for years and cause no illness whatever, but copper coins undergo corrosion in the stomach or intestine and in time cause serious illness or death. In the case of pins, hat pins, and needles, their presence in the stomach is often unsuspected until an abscess forms either in the esophageal region, be- 
tween some of the posterior ribs, or in the flank. The history of the case should be obtained and the habits of the animal inquired into, to determine whether the patient has actually been seen to swallow something, or is in the habit of picking up and swallowing odds and ends. The most prominent symptoms are attempts at vomiting, poor appetite. general dullness and depression, irregularity of the bowels, blood in the feces, general unthriftiness, and emaciation.

An examination with the Röentgen rays makes the diagnosis more positive. A careful digital examination should also be made through the abdominal walls, but this is very often negative.

Treatment.-In the case of small articles, such as needles or small nails, the animal should be fed on soft or doughy food in order to inclose the foreign body during its passage along the intestine. Thick porridge is of good service here. An emetic of 1-20 to 1-10 grain of apomorphin often proves effectual in expelling blunt objects. If the emetic is unsuccessful, a dose of castor oil should be administered.

In the case of hat pins, needles, meat skewers, or similar articles it is better to await developments, since in the course of a few days the point will often work out, causing an abscess. When this is lanced, the foreign body can be grasperl and withdrawn; if a hat pin, the head is cut off with wire cutters and allowed to fall back into the stomach; the abscess cavity must be treated antiseptically. The patient usually makes a rapid recovery. There is some risk of peritonitis, due to these substances perforating the stomach, but it is remarkable how seldom this occurs. As a rule adhesion takes place between the stomach and the peritoneum, thus cutting off the peritoneal carity from infection. Where large sub)- 
stances, such as stones, rubber balls, or large nails, have been swallowed, and the diagnosis is positive, gastrotomy should be performed without delay. A description of this operation will be found on page 207.

\section{Intestinal Catarrh}

Intestinal catarrh is frequently associated with gastric catarrh, the etiology being the same. Intestinal parasites also play an important part in causing catarrh of the intestines. It is also seen as a complication or accessory ailment in distemper.

Symptoms.-There may be romiting, but not sis persistent or so well marked as in gastric catarrh. The appetite is impaired, the temperature is nearly always fairly high, even up to 103 or 105 degrees Falrenheit. The most prominent symptom of intestinal catarrh is diarrhea, the severity of which may vary considerably; in mild cases the feces are pulpy in consistency, while in severe cases there are often slimy, icteric, bloodstained, and usually very fetid feces. Considerable pain and straining accompanies the act of defecation. There is usually an abnormal amount of fermentation, with consequent flatulence. In protracted cases, emaciation is well marked, and the animal presents a forlorn appearance, with tucked-up abdomen, rough, unthrifty coat, and with its tail and hind quarters soiled with feces.

Treatment.-Dietetic treatment is most important, and in mild cases is often all that is required. A spare diet of finely chopped raw meat, continued for several days, is the best. Above all, the immoderate consumption of water must be guarded against. If the appetite is entirely suspended, a bitter tonic should be given to stimulate it. At the outset of the disease a moderate dose of calomel may be given, followed by intestinal antiseptics and 
astringents,-salol and bismuth subnitrate, creosote, or the sulphocarbolates of zinc, calcium, and sodium, combined with bismuth salicylate. Where there is much pain and tenesmus, chlorodyne, laudanum, or Dover's powder* may be given. In cases which exhibit great weakness, alcoholic stimulants, such as brandy and port wine, must be given in conjunction with strychnin or nux vomica. The after-treatment consists in a generous but non-irritating diet, and tonics.

\section{Colic}

Colic is the term here used for abdominal pain, which is of frequent occurrence in canine patients. The causes are various, the most common being flatulence, distension of the stomach or intestine, parasites, foreign bodies, violent and irritant poisons, hernia, intussusception, and volvulus.

Symptoms.-The attack is sudden, the animal is very restless, yelping and crying out, and tries to hide itself. The abdomen is distended, the pulse quickened, and the temperature raised. Vomiting may occur if the dog takes food between the paroxysms, which sometimes he will do. Eventually, if no relief is afforded, excessive prostration ensues, followed by death.

Treatment.-This will depend largely upon the cause, and may be medical or surgical, as the case demands. If parasites or a foreign body in the stomach occasion the colic, an emetic should be given in the attempt to remove the cause. Hot fomentations should be applied externally, and a diffusible stimulant, such as ether, ammonia, or turpentine, given by the mouth.

To combat the pain one-fourth grain of morphin

*The formula of Dover's powder is: Ipecac 10; powdered opium 10; milk sugar S0. The dose is two to twenty grains. 
may be given hypodermically, or chlorodyne or laudanum may be given orally. If the tympanites is very severe and persistent the stomach or the bowel must be punctured with a trocar and cannula. If these measures do not give relief within three or four hours, an exploratory laparotomy should be performed without further delay; further surgical procedure depends upon the condition found. Quite a number of cases reveal nothing, but for some unexplained reason the operation nearly always gives relief from further vomiting and pain.

\section{Intussusception}

The commonest varieties of this condition are the ileocecal and the enteric. In the former the ileum is invaginated into the colon; in the latter the small intestine is protruded into itself. In some cases the invagination may be so extensive that the bowel protrudes through the anus. It is most commonly seen in puppies and young dogs, but may occur also in adults.

Cause.-Intussusception is produced by a violent peristalsis set up by some irritant, such as intestinal worms, indigestible food, or drastic cathartics.

Symptoms.-The patient is seen to strain continually, as if trying to defecate, but only a little fluid or dark, blood-stained mucus comes away. Colicky pains develop later, and vomiting occurs after the condition has lasted for a few hours. Except when straining the patient is quiet, tries to hide away, and shuns companionship. The eyes are brighter than normal. Examination of the abdomen by manipulation between the fingers and thumb reveals a sausage-shaped swelling, which in the early stages is not sensitive to pressure. 
Treatment.-Unless relieved, gangrene of the bowel, peritonitis, and death are the usual ending, although occasionally sloughing of the intussuscepted gut, with adhesion of the serous surfaces, takes place, the "cast" of the bowel being passed per anum. It is, however, not justifiable to rely upon this remote contingency, and immediate operation should be practiced. The prognosis is good if operation is not delayed until adhesions have formed or tissue changes taken place, or until the patient is moribund.

Operation.-Perform laparotony (this operation is fully described on p. 205) and replace the gut into its natural position by manual manipulation. The after-treatment is to keep the patient quiet for some days and on a liquid diet, the bowels being "rested" by the use of morphin.

\section{Diarrhea}

Diarrhea consists of the frequent passage of liquid feces, often offensive and accompanied by pain and straining.

Cause.-Diarrhea maý be a symptom of intestinal catarrh or of distemper, but it is more often caused by irritant food, chills, intestinal parasites, injudicions use of purgatives, or contaminated water.

Symptoms.-The evacuations are generally painful; frequent, and fluid, and have a fetid odor; thirst is increased, but the appetite is often unaffected. If untreated, the animal soon becomes unthrifty, emaciated, and may even die from exhaustion.

Treatment.-If dietetic errors are suspected a full dose of castor oil should be administered, follower by chlorodyne or laudanum combined with an in- 
testinal antiseptic, astringent, and seclative as in the following:

I;

Bismuthi subnitratis ...........drs. 2

Ac. nitrici dil................... drs. 11/2

Chlorodyne ...................... 11/2

Tr. catechu .................drs. 3

Aquæ, q. s......................zs. 4

M. Give a half-ounce in water three times daily.

Or

Ii

Tr. kino,

Tr. catechu,

Tr. opii camphoratæ, aa...........drs. 4

Misturæ cretæ, q. s................... 4

N. Give one dram every three hours.

Another prescription which gives good results ir persistent diarrhea is as follows:

Ac. sulphurici aromatici,

Tr. opii,

Spts. camphoræ, aa..............drs. 2

Aquæ cinnamonii, q. s............... ozs. 4

M. Give one dram three times daily.

If parasites are the cause, a vermifuge must be administered, followed by a laxative and any of the above anti-diarrheal mixtures.

Where there is much fetor, zinc sulphocarbolate is particularly indicated and must be pushed until the dejections resume their normal color.

The diet must be non-irritating, raw meat being best. Milk and lime water is admissible, but large quantities of water must not be allowed.

In persistent and protracted cases stimulants may be needed and strychnin or nux romica-should be given to restore the normal tone of the bowels. Astringent enemas have many advocates, but as they produce much straining and discomfort, and in reality reach only a very short distance up the 
bowel, they are practically valueless, except in the treatment of ulcerative conditions of the rectum. when a five- to twenty-per-cent solution of nitrate of silver, or better of protargol, may be used.

\section{Constipation}

Constipation is the opposite condition to diarrhea, being a partial or complete absence of defecation.

Cause.-Old age and debility, paralysis of the bowels, improper diet, mechanical obstruction either by foreign bodies or by masses of indigested food, hair, and bones are the commonest causes of constipation. Hemorrhoids and engorgement of the anal pouches or glands are further causes of this condition. Constipation may also be a symptom in various febrile disorders, such as distemper, icterus (caused by absence of bile), peritonitis, or enteritis.

Symptoms.-The chief symptom is the more or less complete suppression of defecation. The patient makes continual efforts, but is either unsuccessful or passes only small, dry, single balls of excrement, which are often composed of undigested portions of food and mixed with hair or pieces of bone. Sometimes it is blood-stained. At first the appetite is only slightly impaired, but later it becomes almost or entirely suppressed. Peristalsis is absent; the abdomen may be distended with gas, but more often it is tucked up and hard. On palpation a hard, sausage-shaped mass may be felt in the abdomen, sometimes extending as far forward as the ensiform cartilage. Manipulation is usually painful. The temperature is raised slightly, and upon introducing the oiled finger into the rectum the accumulated fecal mass may be felt. The animal is depressed, and may have colicky pains. There is a peculiar carriage of the tail characteristic of this ailment. 
Treatment.-This depends largely upon circumstances. In the constipation found in the course of fevers the condition does not get so far as the formation of the "fecal tumor" above described, and a mild laxative is all that is required in ordinary cases.

Foreign bodies may require surgical interference if laxatives do not bring about the desired result, but in no instance should drastic purgatives be given. In peritonitis and enteritis, if the case demands the correction of the constipation, enemas alone should be relied upon to empty the bowels.

In old, debilitated, and improperly fed dogs, where the fecal tumor is well marked and there is marked absence of peristalsis (paralysis of the bowel), smal! repeated doses of olive oil and 1-100 to 1-60 grain of strychnin must be administered. Enemas of warm oil should be injected three or four times daily to soften the impacted mass, and the abdomen gently massaged to stimulate peristalsis. Where the impacted mass can be reached, it must be removed piecemeal, either by the finger, a rectal spoon, or, best of all if available, by an instrument known as French's rectal curette, which is a blunt curette having a hollow shaft and an opening in the spoon portion. A rubber tube is attached to the shaft and a continuous flow of water, obtained from an elevated douche can, washes away the particles removed by the curette. Much patience and gentleness of manipulation is required to avoid injury to the walls of the rectum, but the operation must be repeated and persisted in until all the impacted mass is removed. After recovery the dog should be fed on lean meat, free from bones, milk, and beef tea. Regular exercise should be insisted upon, and the animal given a good nerve tonic. In cases that do not yield to the above treatment laparotomy 
may be performed under rigid antiseptic precautions, and if the condition of the impacted bowel allows, the mass must be gently broken up and pressed toward the rectum. If, however, the bowel is inflamed or necrotic, resection of the affected portion must be performed and the ends joined by either of the methods given in the description of that operation, which is discussed in Part II, Section IV (see p. 209). The prognosis, of course, is graver where resection has to be resorted to; still, recoveries do take place, and the operation should be tried in the effort to save the animal's life.

\section{Typhlitis}

Typhlitis, or inflammation of the cecum, is of more frequent occurrence than is usually supposed. It is caused by constipation, indigestible foods, or by microörganisms. The symptoms are acute and persistent abdominal pain, especially after a meal. There is pain on pressure over the posterior portion of the abdomen, and usually the swollen cecum can be felt through the abdominal walls.

Treatment.-All food must be withheld, and only water, or milk and lime water, allowed. A mild laxative to empty the bowels, assisted by rectal injection of oil or warm soapy water, should be administered. One-fourth grain of morphin, hypodermically injected to relieve pain, and hot applications to the abdominal region should be followed by gentle massage over the cecum. Should the above treatment, fail to give relief in twelve hours laparotomy must be performed, with the usual precautions, and the cecum emptied by gentle massage. The after-treatment consists of rectal feeding for three or four days, when the paticnt may be allowed liquid food in small quantities until the abdominal wound is healed. The diet should be of an easily 
digestible and non-irritating nature for a fortnight longer, and bones should not be allowed for at least six weeks.

\section{Enteritis}

Enteritis, or inflammation of the bowels, is an exceedingly fatal disease, and if at all extensive, recovery is doubtful.

Cause.-Among the many causes leading to this grave condition are poisons-either chemical or bacterial-foreign bodies, intussusception, and intestinal parasites-consequently both symptoms and trcatment will vary somewhat in accordance with the causative factor.

Symptoms.-In enteritis produced by poisons the onset of the attack is sudden, the pain continuou'; and severe, and the abdomen is exceedingly tender on pressure and in many cases tympanitic. Peristalsis may be completely in abeyance or there may be profuse and bloody diarrhea. Upon examination the lips, tongue, and mucous membrane of the mouth and jaws are found to be intensely inflamed, sometimes eroded and hanging in shreds. The animal evinces great depression and a disposition to hide away in dark corners and secluded places. Convulsions may supervene, in one of which the animal may die, or it may gradually lapse into coma and die unconscious.

The treatment in these cases consists in emptying the stomach either by the stomach pump or by an emetic (1-20 to 1-10 grain of apomorphin), and in administering the suitable antidote to the poison. This having been done, the inflamed surfaces of the stomach and bowels must be soothed with demulcents, such as barley water, linseed tea, or marshmallow tea, and protectives given, such as full doses of bismuth subnitrate. To relieve the agonizing 
pain hypodermics of one-fourth to one grain of morphin sulphate, or the H-M-C tablets (No. 1) may be given as required.

Hot applications to the abdomen afford relief and if necessary the patient's strength should be supported with stimulants, such as ether or brandy administered hypodermically. In cases that respond to treatment and become convalescent the diet should be restricted at first to milk, gruel, and beef tea. Later on, very small quantities of soft and non-irritating food may be given.

When enteritis is due to any of the causes above mentioned other than poisons, the attack usually comes on more slowly and as a sequel to other preliminary disorders. The animal becomes more depressed, the nose is hot and dry, the temperature extremely high, 105 to 107 degrees Fahrenheit, and the pain continuous. The abdomen is tender, frequently tympanitic, and sometimes hard and tense. Peristalsis is absent, and constipation obstinate. The animal evinces intense thirst, but the stomach is su irritable that vomiting is easily cxcited, even by water. Symptoms of collapse appear, the pain abates, the pulse is barely perceptible, the mouth feels cold and clammy, and the patient dies in comi or, more rarely, in convulsions. In all forms of enteritis extreme fear and apprehension are evident, the patient presenting a haggard, miserable, and appealing appearance.

Treatment.-Enteritis following intussusception, impacted foreign bodies, and so on, is almost invariably fatal; operation offers the only hope for recovery, and that is most forlorn. In other cases, however, anodynes and hot applications to the abdomen to relieve pain, with demulcents to protect and sooth the inflamed surface, enemas to gently solicit action of the bowels, and stimulants as re- 
quired, constitute the line of treatment to be followed. The after-treatment as regards diet is as before mentioned.

\section{Tumors}

Neoplasms of the intestines are fortunately of rare occurrence in dogs, as the diagnosis is almost impossible, but their presence may be suspected in cases where the digestive functions are disturbed and continual attacks of pain, especially after meals. and rapid emaciation ensue. Their presence may sometimes be diagnosed either by palpation through the abdominal walls in thin subjects or by the aid of the Röentgen rays. The varieties found are sarcomas and carcinomas. 


\section{SECTION VII}

\section{DISEASES OF THE RECTUM AND ANUS}

THIS class of ailments includes hemorrhoids, atresia ani, and obstruction of the anal glands.

\section{Hemorrhoids}

A dilated and varicose condition of the veins around and just within the anus is known as hemorrhoids. This troublesome condition is frequently seen in old, overfed, and fat dogs.

Cause-Constipation, overfeeding, debility, impaired portal circulation, old age, and want of exercise are the major causes of hemorrhoids in dogs.

Symptoms.-Pain and irritation during defecation and pruritus, the animal continually licking the parts and rubbing them on the ground, are evidences of hemorrhoids. The anal surface appears moist and swollen and the feces are frequently streaked with blood. Constipation is often present both as a symptom and as a cause, the patient, owing to the pain experienced, delaying the defeca. tion as long as possible.

Treatment.-This consists in attention to the state of the bowels, mild laxatives such as cascara, phenolphthalein, or olive oil being given to effect, and local treatment to the affected parts. The anal region should be kept clean and dressed with anodynes and astringents, such as adrenalin ointment, hazelin ointment, bensinol ointment or the Unguentum Gallae et Opii (B. P.). In internal piles suppositories of any of the above may be 
used so as to bring the medicaments into direct contact with the dilated veins. In cases where the above treatment is ineffectual a resort must be had to surgical interference. The piles may be removed either by ligation or by clamps and the actual cautery. By the former method, the piles are picked up separately with forceps and ligated by passing around them a curved needle armed with the ligature, after first cutting a groove around the base of each with blunt-pointed scissors. Where the clamp is used the pile is picked up as before, the clamp applied, and the loop removed with the cautery. In operating by either method a general anesthetic is advisable.

After-treatment.-After the operation the patient should be kept without food for twenty-four hours and for the next week allowed only small quantitie.s of milk, beef tea, or other sloppy diet. Small doses of opium should be given three times a day to keep the bowels at rest, but if no movement of the bowels has taken place by the fourth day a warm enema of oil should be given. Locally, the parts must be kept clean and dressed with antiseptics, such as chinosol ointment or suppositories.

\section{Prolapsus Recti}

This condition generally occurs suddenly during the course of or after recovery from some debilitating illness.

Cause-Debility, constipation, diarrhea, hemorrhoids, rectal irritation and the consequent straining are all important causes of prolapsus recti.

Symptoms.-The patient shows a disposition to hide, and is quieter than usual. The floor on which it has lain down is blood-stained, as are also the hind quarters. Examination, which is resented, reveals the prolapsed gut, congested and, it may be, 
lacerated. If not treated, the gut in a. few hours becomes intensely swollen, of a dusky hue, and excecdingly painful, and if further neglected death may take place from shock, collapse, or septic absorption. When the act of defecation takes place the gut is seen to protrude and retract as straining takes place and ceases.

Treatment.-As a rule a general anesthetic should be given, since the parts are very sensitive and there is always straining by the patient; furthermore, reposition is a great deal easier when the parts are relaxed and the patient's opposition under control. In recent cases before the mucous membrane of the gut has become friable and easily lacerated, the parts should be bathed freely with hot water, to which may be added some antiseptic such as creolin or chinosol, or if exceedingly congested. they may be sprayed with a solution of adrenalin chlorid, 1 to 10,000 or 1 to 20,000. The gut is then cautiously manipulated with the fingers and the prolapsed portion replaced, the rectum being smoothed out into its normal position with the middle finger. A stitch or two should then be placed across the anus to retain the bowel, or a pursestring suture inserted for the same object.

In longer standing cases in which the prolapsed portion is lacerated and the mucous membrane friable, cold water should be substituted for hot in the preliminary bathing as it will tone up the parti and render them less liable to injury during the subsequent manipulations. Straining must be controlled by morphin or cannabis indica, and the diet restricted to slops. Constipation should be guarded against by the use of laxatives of olive oil or cascara. Prolapse of the rectum is very prone to recurrence, and amputation will very likely be necessary to obtain permanent relief. Amputation should 
be proceeded with in the first instance if the parts are badly torn or show symptoms of gangrene.

\section{Amputation of the Prolapsed Rectum}

Instruments.-Scalpel, scissors, tissue forceps, eight needles and sutures, a needle holder and artery forceps are the instruments needed for this operation. An antiseptic solution (chinosol 1-1000) must also be provided.

Operation.-The instruments, needles, sutures, and the operator's hands, having been made surgi. cally clean and the patient under a general anesthetic, H-M-C and choloform by preference, gentle pressure is made of the prolapsed gut and a needle armed with a suture (catgut or absorbent silk) is thrust through the gut from above downwards as close as possible to the anus; another suture is placed transversely so as to cross the first at right angles. The assistani then holds the ends of the sutures while the operator amputates the gut close behind the sutures. Any hemorrhage is then controlled with the artery forceps and the sutures cut at the point of intersection and tied. In this way it is seen that the remaining portion of the rectum is secured with four sutures. Sutures an eighth of an incl apart are then put in all round the gut, the ends cut short, and the parts gently pushed back inside the sphincter ani after being washed in the antiseptic solution.

After-treatment.-All food must be withheld for twenty-four hours and the diet restricted to slops for at least four days, the bowels up to that time being kept quiet by small doses of opium. After the fourth day, if the patient is uncomfortable, a laxative may be given to produce action of the bowels, and for a month at least the feces must be 
kept soft by the administration of laxatives (olive oil) and soft foods.

\section{Prolapsus Ani}

The causes of this condition are the same as those producing prolapsus recti, and while not of so serious a nature as that condition, it is often exceedingly troublesome to deal with and causes the animal much discomfort, to say nothing of the unsightly appearance it presents. The symptoms are practically the same as in prolapsed rectum except that only a very small portion of the posterior part of the rectum appears as rather a red, angry-looking mass at the anal opening.

Treatment.-Reposition is comparatively easy. but to induce the parts to remain in their natural position is often quite another matter. Astringent washes may be tried and the anus sutured, the same rules as to diet and regulation of the bowels being applied as in prolapsus recti.

If these measures fail to give permanent relief, operation should be resorted to. The animal should be placed on a milk or beef-tea diet for a few days previous to operation and should receive a laxative to clean out the bowels. Immediately before operation the patient should receive an enema, of warm boracic acid solution, and a general anesthetic administered. The relaxed portion of the mucous membrane is then gently pulled out from the anus by the fingers and three or four elliptical wounds, from one-half to one inch in length, made witis sharp scissors in the longitudinal axis of the mucous membrane close to the anus. The rationale for this operation lies in the fact that contraction takes place during cicatrization and thus tends to prevent the parts from again prolapsing.

The after-treatment consists in giving antiseptic 
injections or suppositories, keeping the bowels at rest by means of opium, and prescribing a sloppy diet for a week or two. The animal should then be placed on a substantial, nutritious diet and every effort made to improve the general condition by proper exercise and a good course of tonic medicine.

\section{Fistula in Ano}

This is a fistulous opening in the anal region.

Causes.-Laceration of the rectum by foreign bodies, such as needles, fish bones, or spicules of bone, with subsequent abscess formation, neglected hemorrhoids, and constipation are common causes of this ailment.

Symptoms.-The patient is continually licking the anal region and demonstrating the irritation that exists there by rubbing it along the ground. Upon examination a fistula, discharging pus, is seen near the anus.

Treatment.-The extent and depth of the sinus must be accurately determined by aid of a probe gently and cautiously introduced. By means of a grooved director and bistoury the sinus should then be laid open, cutting through all tissues, even the sphincter ani if necessary, in order to provide thorough drainage. The abscesses must be evacuated, the fistulous walls curetted, and the wound tamponaded with iodized gauze, which should be removed within twenty-four hours. The wound is then irrigated with antiseptic lotions until healed. A new line of treatment for fistulæe and similar conditions has lately come into rogue with remarkably good results. The fistulous tracts are first syringed out with hydrogen peroxid and as much pus as possible removed; a mixture of bismuth subnitrate and paraffin is then injected, so as to com- 
pletely fill the whole tract, and the opening plugged to prevent the escape of the mixture.

The formula for this bismuth-paraffin paste is as follows :

Bismuth subnitrate ..........120 grams

White $\operatorname{wax} \ldots \ldots \ldots \ldots \ldots . \ldots 20$ grams

Paraffin .................20 grams

White vaselin ...........240 grams

The mixture is heated to liquefaction before being used and is injected by a metal syringe that has been previously warmed. The treatment may be repeated every five days as long as necessary.

\section{Atresia Ani}

Imperforate anus is a congenital defect and often escapes notice for some hours after the puppies are born. Upon examination the anal opening is found to be absent, but the position of the anus may be found by a bulging of the perineum caused by the imprisoned feces. In some cases the opening is merely occluded by the skin and all that is required to rectify the condition is the excision of an elliptical portion of the integument. In others, however, the rectum ends as a blind tube and is not attached to the anus proper. When such is the case, after removing an elliptical portion of the skin the end of the rectum must be sought for, brought back into the wound, opened, and stitched to the raw margin of the wound. Occasionally the rectum is entirely absent, and of course treatment is then hopeless.

The after-treatment consists in the application of antiseptics and keeping the parts clean.

\section{Obstruction of the Anal Glands}

Situated on either side of the anus, the ducts communicating with the rectum just anterior to the 
sphincter ani are two pouches whose walls secrete a mucoid substance, the function of which is to lubricate the parts during the act of defecation. If by reason of any rectal irritation the ducts of these glands become blocked the secretion eventually causes them to become enlarged and encroach on the lumen of the rectum, causing difficulty and pain during the act of defecation.

Symptoms.-A swelling about the size of a walnut on either side of the anus, constipation, often the result of the pain which defecating causes, and irritation shown by the animal's licking the anal region and rubbing it along the floor are indications: of this ailment.

Treatment.-In many cases the evacuation of the contents of the glands by pressure exerted by the finger introduced into the rectum and the correction of constipation and any dietary errors are sufficient to bring relief, but if the condition continues, more radical measures must be adopted. The glands should be opened freely from the exterior, the contents cleaned out, and the walls freely curetted and then painted with tincture of iodin to destroy their secreting properties. The wounds are then treated antiseptically until healed. The operation is quite safe, and if care is taken to entirely destroy the secreting walls a permanent cure may be confidently expected. 


\section{SECTION VIII}

\section{DISEASES OF THE LIVER \\ Icterus or Jaundice}

TCTERUS cannot properly be called a disease per 1 se, but is really only a symptom of some other disease affecting the liver or its ducts. Since diverse causes may produce icterus, it is necessary for purposes of clinical description to classify the various forms of icterus according to their etiological factors.

\section{Catarrhal Jaundice}

Catarrhal jaundice occurs usually in connection with gastroduodenal catarrh, the catarrhal condition extending up the ductus choledochus, the duct becoming blocked by the subsequent swelling and formation of mucus. The bile is thus prevented from reaching the bowel and dams back to the liver, where it is reabsorbed into the circulation.

Symptoms.-In addition to the symptoms appearing in the course of an attack of gastroduodenal catarrh, the visible nutcous membranes are found to be stained a characteristic yellow color. Later on bile may be found in the saliva, urine, and milk of nursing bitches. Lastly the skin becomes deeply stained, and if the stenosis of the duct is complete cholemia supervenes with a fatal termination. Its advent is recognized by great weakness, fall of temperature, slow, weak pulse, and increase of icteric symptoms. The patient dies in coma. In addition to the foregoing signs, the absence of the bile in the intestinal tract scrionsly interferes with the digestive processes, peristalsis is diminished, and the 
feces become clay colored and very offensive. Constipation is usual. Catarrhal jaundice is the most important jaundice of the dog, and the prognosis is usually grave.

\section{Heptogenous Icterus}

This form of icterus is seen as a symptom in hepatitis, atrophy of the liver, degenerations of the liver, neoplasms, and thrombosis of the portal vein.

\section{Hematogenous Icterus}

Hematogenous icterus is jaundice occurring in the course of septic or infective diseases. It is due to decomposition of the blood.

\section{Malignant Malarial Jaundice}

This form of jaundice is a symptom of canine malaria (caused by the Piroplasma canis).

Treatment.-One of the most essential factors in the treatment of catarrhal jaundice is the proper regulation of the diet. Lightly cooked or raw meat, finely minced and mixed with small quantities of boiled rice or oatmeal, form the best ration. Constipation should be overcome by moderate doses of calomel followed in a few hours by a saline laxative such as Epsom salt or artificial Carlsbad salt.

The abdomen over the region of the liver should be gently massaged, as the gentle pressure helps to mechanically empty the gall bladder. Boldin, the active principle of Peumus boldus, gr. 1-30, bilein gr. 1-8 combined with hyoscyamine gr. 1-250, should be given three times daily. In cases with great depression and threatening coma, recourse must be made to stimulants such as strychnin, caffein, camphor, and so on.

In the heptogenous form the icteric symptoms are best treated with small repeated doses of calomel, 
followed by nitromuriatic acid. Other treatment must be directed against the primary condition.

Hematogenous icterus must be treated by vigorous eliminative measures combined with proper treatment of the condition responsible for it.

\section{Hepatitis}

\section{(Inflammation of the Liver)}

Hepatitis occurs in two forms, acute parenchymatous and chronic interstitial.

Cause.-Acute parenchymatous hepatitis, inflammation of the liver substance, occurs during the course of the various infectious diseases. Injudicious feeding on rich, stimulating food, exposure to cold, and phosphorous poisoning are also causes of hepatitis.

Symptoms.-Vomiting, colicky pains, pain on pressure over the hepatic area, icterus and fever are evidences of hepatitis.

Treatment.-Administer small doses of calomel followed by saline laxatives, hot applications to the abdomen and hypodermic injections of morphin as required to relieve pain. The diet should consist of sloppy food during the attack, and previous errors corrected thereafter.

\section{Cirrhosis of the Liver-Interstitial Hepatitis}

The most marked result of chronic interstitial hepatitis is the formation of new connective tissue in the liver. The character, amount, and distribution of the new tissue varies greatly in different cases. Secondarily there are usually marked changes in the liver cells, blood vessels, and gall ducts. The new tissue is most commonly formed and most abundant in the periphery of the lobules along the so-called capsule of Glisson, but it may extend into 
the lobules between the liver cells. The new formed tissue tends to contract, causing atrophy of the celli from pressure or from interference with the portal circulation. The bile ducts may also become obliterated and both the secretion and the outpouring of the bile be interfered with. Owing to the above mentioned changes, secondary lesions occur; these are ascites or dropsy icterus, gastrointestinal catarrh, and hemorrhages.

Cause.-The causes of cirrhosis in the dog are unknown, but it is often seen in connection with cardiac diseases.

Symptoms.-In cirrhosis of the liver gastrointestinal catarrh with icterus, abdominal dropsy, cardiac disturbances, and gradual emaciation give evidence of the disease.

Treatment.-Cirrhosis of the liver invariably sooner or later ends in death, and treatment is ineffectual. However, life may be prolonged by treating the symptoms as they arise, and by liberal diet, alteratives and tonics keeping up the patient's strength.

\section{Ascites-Abdominal Dropsy}

The accumulation of fluid in the abdominal cavity is always a secondary condition. The fluid in true ascites is a non-inflammatory transudate of serum from the peritoneal blood vessels and must be distinguished from the inflammatory exudate formed in peritonitis. The fluid is generally straw colored, though sometimes it has a reddish tinge; it is sticky to touch and clots readily on exposure to the air. It is usually clear, but may contain shreds of fibrin. Contrary to the exudate of peritonitis, it is rich in fibrinogen but poor in cells.

Cause--Cirrhosis of the liver, on account of the obstruction of the portal circulation, for the 
same reason new growths of the liver or the peritoneum are etiological factors; as are also renal disease and cardiac disease.

Occasionally ascites appears after an' attack of distemper, there being no organic changes discernible. Such cases yield readily to treatment and make permanent recoveries. Recovery in cases due to primary cardiac, renal, or hepatic disease will depend upon the extent and amenability to treatment of the primary cause.

Symptoms.-This disease is manifested by a gradual enlargement of the abdomen, pendent belly, and a characteristic falling in of the flanks. Palpation with the finger tips and auscultation reveal the presence of fluid within the cavity. In great accumulations of fluid the pressure upon the diaphragm and lungs causes much difficulty in respiration, sometimes even to suffocation. The appetite is variable, the secretion of urine diminished, and constipation usually present. The conditions which may simulate ascites and which may be mistaken for that condition are:

1. Chronic Peritonitis.-The history of the case, the absence of cardiac, renal, or hepatic disease, and the character of the fluid, determined by examination of a sample obtained by aspiration with a fine needle or trocar, will, however, serve to make the distinction.

2. Pregnancy--Palpation of the abdominal walls and contents reveals the true nature of the enlarged abdomen.

3. Distended Bladder.-The shape of the abdomen is different, and dyspnea is not increased by elevation of the hind quarters as it is in abdominal dropsy.

4. Urine in the Abdominal Carity From Rup- 
Inred lindeler.-Lrine in the abluminal cavity from ruptured bladder is a condition in which there are always symptoms of collapse, absent in ascites. Aspiration reveals urine instead of ascitic fluid.

Treatment.- Mleviation and sometimes permanent relief may be obtained for this condition by (1) purgatives: (2) diuretics; or (3) paracentesis abdominis. In addition treatment against the primary cause must, however, be instituted, and even then in many cases recurrences occur. It is usual, however, in addition to treating the primary cause to make use of all three of the above-named methods of treatment for relieving the ascitic symptoms.

1. Drastic purgatives are here indicated: Elaterin in doses of 1-20 to 1-12 grain or one to three grains of colocynth are the most useful. These cathartics cause copious fluid evacuations.

2. Digitalis, potassium iodid, potassium asetate, or spirits of juniper are the usual diuretics employed.

The following is a useful formula for a diuretic pill :

R

Scillæ,

Digitalis foliæ,

Ext. hyoseyami, aa.................. 1

Calomel ......................gr, 1-4

Misce et fiat pilula mitte No, XII

sig.-Give one pill three times a day to free elimination.

3. The evacuation of the fluid by means, of the trocar and cannula-paracentesis abdominis. (The technic for this operation will be found on p. 204.) This operation should not be delayed if there is excessive dyspnea, and it may repeated as often as required. The diet should consist of meat extracts, milk, and finely minced meat. Water should be allowed only in small quantities. The patient should 
afterwards receive a good course of tonic treatment.

\section{Diabetes Mellitus}

This is a condition in which the urinary secretion is not only abnormally increased but also contains a large quantity of sugar.

Cause.-This disease is essentially one of perverted hepatic metabolism, where glycogenic function is interfered with by the absence or poverty of the internal secretion of the pancreas. Due either to disease of or to a lack of function of the islands of Langerhans in the pancreas, this internal secretion is diminished or suppressed and its absence from the blood stream causes the perverted metabolism in the liver. Sugar in the urine is also found, after re. moval of the pancreas, in concussion of the brain, fracture of the skull, epilepsy, urari poisoning, and after large doses of morphin and amyl nitrate.

Symptoms.-Diabetes mellitus is evidenced by depression, dullness, and great emaciation, although the appetite is enormously increased. Thirst is increased and the patient passes large quantities of urine of high specific gravity, 1,035 to 1,060 , containing from seven to twelve per cent of sugar.

Ulceration of the cornea and blindness may occur from cataract formation, and a chronic bronchitis is also often associated with this condition. Diarrhea is usually persistent. The course of the disease is gradual, the animal slowly wasting away, finally lapsing into coma in which it dies. Sometimes toward the end the comatose state is ushered in by convulsions. The prognosis is unfavorable.

Treatment.-The diseased animal should be given a meat diet with as little carbohydrates as possible in cases of diabetes mellitus. Thie administration of arsenic in the form of Fowler's solution is the most 
effectual form of medication in checking the formation of sugar in the liver. Glycerin also has the same property of regulating the glycogenic function of the liver and may be given in conjunction with the arsenics.

In impending coma, large doses of sodium bicarbonate must be given, and if coma has supervened 50 to 100 cubic centimeters of a saturated solution of the same salt should be given intravenously.

The treatment that has up to the present time given the most favorable results is the administration of an extract of pancreas containing all the ferments combined with arsenic and bromid of gold. This extract, obtainable under the name of trypsogen jambul (Eugenia jambolana), also has the property of arresting the formation of sugar, and may be given in doses of from five to ten minims of the fluid extract or five to ten grains of the powdered seed. It may also be given with good effect combined as follows in tablet form:

Antipyrin $\ldots \ldots \ldots \ldots \ldots \ldots \ldots$. . . . . .

Fluid extract jambul............... ii

Codein sulphate..................... ss

Give one such tablet, two or three times a day or oftener, to maintain effect. 


\section{DISEASES OF THE KIDNEY}

DISEASES of the kidney in the dog are of relative unimportance owing to their rarity. For clinical purposes they may be classed as follows: acute nephritis, chronic nephritis, suppurative nephritis, pyelitis, degenerations, neoplasms, and parasites.

\section{Acute Nephritis}

\section{(Acute Infiammation of the Kidney)}

Cause-Acute nephritis occurs from various causes. It may arise from blows and injuries to the back or from exposure to cold. Or it may occur during the course of infections diseases, such as distemper, bronchitis, septicemia, and pyemia, which fact is not remarkable when we remember that many bacteria and their toxins are excreted by the kidneys. Nephritis also follows poisoning by arsenic, mercury, phosphorus, lead, male fern, squill, turpentine, croton oil, cantharides, tar, phenic acid, ergot, and other poisons. It may also arise from infection extending from the pelvis of the kidney (pyelitis), the ureters, or bladder (cystitis).

Symptoms.-The most important and in the course of severe infectious diseases sometimes the only symptoms are in connection with the urine. There is well marked albuminuria, the quantity of urine (except in the initial stage, when for a few hours it may be increased) is much reduced, sometimes entirely suppressed for several days. The causes of this anuria are stoppage of the urinary canals by casts, inflammatory swelling of the kidney 
tissues and consequent compression of the ducts, and diminished secretion of water.

The specific gravity of the urine during acute nephritis is higher than normal $(1,035$ to 1,050$)$, and the urine itself is thicker in consistency," slimy, turbid, dark in color, and may be bloofl-stained (hematuria). A microscopic examination reveals the various kinds of casts in large quantities, numerous epithelial cells, and red and white blood corpuscles.

The discharge of urine occurs only in small quantitic: during acute nephritis and is accomplished by considerable pain anci straining. If the bladder is examined per rectum it is found to be empty. Pressure over the loin causes pain, direct manipulation of the kidneys themselves is painful, and they are found to be enlarged.

Renal colic is most marked at the commencement of the disease. The patient arches his back, and the gait is staggering. In male animals one testicle is often drawn up. The appetite is suspended from the beginning and romiting is common. Constipation generally sets in soon after the vomiting appears, to be later alternated with diarrhea.

The temperature ranges from 103 to 105 degrees Fahrenheit. At first the pulse is liard and strong, but later on becomes weak and fast. Hydremic swellings appear on the head, breast, belly, scrotum, extremities and within the abdomen, while abdominal dropsy is also somettimes seen.

In the severer cases, tending to a fatal issue, the symptoms of uremia are added to the foregoing. Uremia consists of an accumulation of urinary constituents in the blood and tissues. The symptoms of this condition are a fall of temperature, romiting. great weakness, dyspnea, convulsions, and coma. The patient exhales a characteristic uriniferous odor from the lungs and skin. 
Treatment.-The treatment of acute nephritis must begin with the prohibition of all foods and drugs having an irritant effect upon the kidneys, and the diet must consist exclusively of milk. The bowels must be kept freely active with catharticsone to two grains of calomel-and the kidneys solicited by non-stimulating diurectic such as five to twenty grains of potassium acetate. If edema is present the potassium acetate may be replaced advantageously by digitalis.

To relieve the congestion warm, moist compresses may be applied to the loins and hypodermic injections of morphin sulphate given. In urgent cases the patient should at once be placed in a hot bath, rubbed dry, and kept warm with flannel coverings. Later on from five to twenty minims of tincture of iron perchlorid with a bitter tonic, such as one half to one ounce tincture of cinchona, may be given with advantage.

\section{Chronic Nephritis}

Chronic inflammation of the kidneys may develop from the acute form or it may assume an insidious chronic form from the beginning.

Cause.-Irritants to the kidney substance such as chemical irritants, bacteria, emboli, and the extension of infection from the pelvis in cases of pyelitis, may cause chronic nephritis where the irritation is not severe enough to produce acute nephritis.

Symptoms.-These symptoms come on so gradually that the disease may be far advanced before it is noticed. The patient exhibits an unexplained lassitude and loss of appetite, and later dropsical swellings appear on the dependent parts and direct one's attention to the urine, which alone can give diagnostic data. 
Upon examination we find that the urine has its specific gravity increased, and contains albumin, epithelial cells, casts, fatty granular cells, and sometimes red blood corpuscles also. The amount secreted is always diminished, although in the dog it is not always easy to get reliable information on this matter.

Upon further examination the pulse is found to be hard and tense and the heartbeats strong and audible on both sides of the chest. The area of cardiac dullness on percussion is found to be increased. All these symptoms point to hypertrophy of the heart, a common sequel to renal disease. The temperature is usually only slightly above normal; thirst is greatly increased. The disease may go on to a fairly favorable termination, its intensity may increase, uremia and death supervening, or again it may pass into the chronic indurative stage.

When chronic induration of the kidney takes place, the urine becomes plentiful, is of a low specific gravity, contains few casts or epithelia, and but little albumin. The dropsical swellings disappear; thirst is very intense and the blood pressure is high. (Compensatory hypertrophy of the heart.) If this compensation is complete the patient may survive for years, but gradually the strain on the heart leads to increasing incapacity of that organ. The pulse grows irregular and feeble, and catarrh of the bronchi, stomach, and intestines sets in, combined with dyspnea. Anemia is a constant symptom. The secretion of urine again diminishes, while the specific gravity and the albuminous content increase. Death finally ensues from uremia.

Treatment.-The treatment of chronic nephritis is much the same as for the acute form, though the prognosis is more unfavorable. Cardiac tonics such as digitalis or caffein must first of all be adminis. 
tered and the bowels kept open with calomel. Potassium iodid should be given for its diuretic and deobstruent properties and the anemia combater with one half dram of tincture of iron perchlorid. The uremic symptoms must be met by drastic purgatives and stimulants. Elaterium is the drug of choice for this condition and should be given in doses of one-fourth of a grain.

\section{Suppurative Nephritis}

This grave condition arises from the invasion of the kidney by pyogenic organisms during the conrse of some other diseases, such as septicemia, pyemia, endocarditis, pneumonia through metastasis, or during purulent cystitis by way of the ureters or from renal calculus. The condition occurs in two forms, the diffuse and localized (abscess of the kidney). In the latter form the whole kidney may become changed, by the complete destruction of its parenchyma, so that its capsule becomes a sac containing nothing but pus.

Symptoms.-These are usually masked by the symptoms of the primary disease, but emaciation, increased thirst, fluctuating temperature, and anorexia point to suppuration and the urine should be examined. The urine is usually offensive, contain's blood and kidney detritus, and is thick and slimy. In thin animals the kidney may be palpated from the exterior; and in small animals it may be palpated per rectum and the condition recognized.

Treatment.-The treatment of this condition is purely surgical and consists of aspirating the pus by puncture in the lumbar region or the extirpation of the affected kidney by laparotomy. This operation is fully described on page 205. 


\section{Pyelitis}

\section{(Inflammation of the Pelvis of the Kidney)}

Cause-Pyelitis is the usual result of an infection spreading from the bladder to the kidney. It also often develops in the course of infectious diseases, and may be cansed by irritant poisons and renal calculi. The presence of the Sitrongylus giga in the pelvis of the kidney may lead to pyelitis. This, however, is of rare occurrence.

Symptoms. The manifestations of pyelitis are so indefinite that we have to rely for its diagnosis almost entirely upon a microscopical examination of the urine. Pus, and the cells characteristic of the epithelium of the pelvis, being found therein are indicative of this condition. In addition we may find coagulie of blood, necrotic mucous tissue, and if Strongylus gigas is the exciting cause, ova of this parasite. There is often difficulty in urination and colicky attacks of pain.

Treatment.-In ardition to the treatment required for the primary discase, antiseptics and astringent: shot1d be administered. Of these formin (hexamethylenetetramin) is most effectual, exerting its antiseptic action by the liberation of formic aldehyd along the whole urogenital tract. In chronic cases buchu and juniper in doses of one to two ounces may be given with benefit. For the pain, give Cannabis indica in doses of from one to two minims of the fluid extract.

\section{Degenerations and Tumors}

Neither of these conditions is of much interest, from a clinical point of view, since in the dog their diagnosis is most nncertain and treatment useless. 


\section{Parasites}

The parasite with which we are chiefly concernel is the Strongylus gigas, a nematode worm usually of a blood-red color, slightly tapering at the extremities. Its length may reach three feet. The ova are oval in shape and brownish in color, appearing granular or pitted on the surface. The diagnosis of infestation by this parasite can be made with certainty by the discovery of the ova in the urine by microscopic examination. Suspicion of this parasitism should be aroused by frequent attacks of renal colic, hematuria, rabiform symptoms, and emaciation.

The treatment consists of repeated small doses, from five to ten minims, of oil of turpentine.

\section{Diabetes Insipidus}

This is a rare disease in the dog, characterized by an abnormal increase of the urinary secretion. It is most frequently found in young animals, and may be ascribed as due to some derangement of the nervous system possibly affected reflexly from the intestinal canal. Pressure on the brain caused by neoplasms has also been noted as a possible cause.

Diabetes Insipidus is classed with diseases of the kidney because an increased functionating of this organ is the most noticeable symptom. It is probable that it should be discussed with diseases of the nervous system.

Symptoms.-Increased thirst, emaciation, and the passage of large quantities of pale-colored urine of low specific gravity characterize this disease.

Treatment.-A meat diet, avoidance of cereals, and exercise are essentials in the treatment. Codein in doses beginning with one third to one grain and 
tincture of valerian, one half to one dram three times daily, are the therapeutic indications.

\section{Diabetes Mellitus}

For a discussion of this disease see the preceding section on "Disease of the Liver." 


\section{DISEASES OF THE BLADDER}

ISEASES of the bladder, while not very common, are numerous, including cystitis, spasm of the sphincter vesicæ, paralysis of the sphincter vesica, lithiasis, retroflexion, new growths, prolapse, and eversion.

\section{Cystitis}

Cystitis consists of a catarrhal inflammation of the mucous coat of the bladder.

Etiology.-Pyogenic bacteria, colon bacilli, and other organisms may gain access to the bladder from the kidneys, during the course of septic diseases, from an extension of an infection in a near-by organ (uterus or vagina) or from the surrounding tissues, and from the introduction of a dirty catheter.

Cystitis may also be produced by chemical irritants, such as cantharides, turpentine, and phenol administered internally or absorbed through the skin or wound surfaces and eliminated in the urine: by irritation of a cystic calculus; by prolonged retention of urine; by hypertrophy of the prostate, and by traumatisms resulting from kicks or being run over.

Symptoms.-The irritable condition of the bladder is shown by the patient's frequent desire to micturate and by the increased amount of urine passed in the twenty-four hours (the normal amount of urine voided in twenty-four hours is seventeen to fifty ounces, depending upon the size of the animal). The amount passed, however, at each act of urination is usually small and often accompanied by pain. 
The urine is turbid and darker in color than normal and is usually alkaline in reaction (normal urine in the dog is acid), although where the colon bacillus is the causative agent the urine is acid. On microscopical examination the urine is found to contain mucus, pus cells, bladder cells, bacteria, and often blood corpuscles. The salts in the urine are alsn greatly increased luring attacks of cystitis, the triple phosphates predominating.

Examination of the bladder per rectum is painful, and in many cases, especially when the disease has become chronic, the walls of that viscus are found to be greatly thickened. If the exciting cause be a calculus, palpation of the bladiler through the rectum will reveal its presence. The temperature as a rule does not run high, the fever being of a remittent type.

The appetite is suspended and the patient suffers from general malaise. The chief complications to be dreaded in cystitis are an extension of the infection up the ureters, with consequent involvement of the kidneys (nephritis or pyelonephritis) and rupture of the bladder from necrosis of its walls.

In chronic forms of cystitis the symptoms are not so evident, the most prominent being incontinence of urine, and the micruscope must be relied upon to complete the diagnosis. The walls of the bladder in these cases, as stated above, are found upon examination jer refum to be greatly thickened.

Treatment.-The treatment of crstitis must be dietetic, medicinal, and local. In every case the diet must be of a non-irritating character, milk being the ideal food. Barley water ad 7ibitum should also take the place of ordinary water, and should be kept constantly before the patient. Among the medicinal agents user for this condition are formin. lithium benzoate, ammonimm benzoate, hyoscyamin. 
potassium bicarbonate, and buchu. Convenient formulas are as follows:

R Lithii carbonas ..................... ii Formin ............................. $v$

Sodii benzoates .................. grs. $\mathrm{v}$

M. et $\mathrm{f}$. tablet No. XXX.

Sig.-Give one tablet in water every three hours.

R Potassii bicarbonas ................ iii Tinct. hyoscyami.

Fl. Ext. Tritici.

Syr. auranti ................... oz. i

Aquæ qs. ad..................... iv

Sig.-Give two drams three times daily.

In chronic cases buchu, copaiba, santal oil, or other stimulating diuretics should be substituted for the above. If pain and spasm are prominent, use 1-200 grain of hyoscyamin, sufficient to control.

The local treatment consists of washing out the bladder, after catheterization, with, first of all, tepid water, and then with a two-per-cent solution of boric acid. If in addition to the other symptoms hemorrhage is constant or severe, the boric-acid solution should be replaced with a 1 to 10,000 solution of adrenalin hydrochlorid. In very acute cases, where there is much pain and tenderness, hot fomentations to the posterior portion of the abdomen gives much relief, and should always be applied.

\section{Spasm of the Sphincter Vesicæ}

This condition more often affects well-broke! house dogs which are not afforded an opportunity of relieving themselves and whose cleanly instincts will not allow them to do so in the house. The long voluntary control over the sphincter funally gives way to uncontrollable spasm, and the patient is unable to urinate when the opportunity arises.

Symptoms.-Repeated but ineffectual efforts to 
urinate, pain, great discomfort, and anxious expression are evidence of the trouble. Upon examination the bladder is found to be quite full and often enormously distended. If not relieved, rupture is almost always the result, although in some cases the patient dies, exhibiting symptoms of uremic poisoning. The symptoms of rupture of the bladder are sudden collapse, constant pain, the presence of fluid (urine) in the abdominal cavity, and upon examination (palpation and catheterization) the bladder is found to be empty.

Treatment.-As soon as spasm of the sphincter vesica is recognized the bladder must be emptied, great care being taken, because of the danger of rupture, that the patient does not struggle. If any difficulty is experienced in passing the catheter the attempt should be abandoned and the bladder emptied by puncturing it through the abdominal wall with a fine trocar and cannula. The puncture is made immediately anterior to the os pubis, just to one side of the penis in the male and in the median line in the female. In any event, when the catheter is being passed or when the trocar is being insertel the patient should be in a standing position.

Hyoscyamin sulphate, 1-60 to 1-30 of a grain, or tincture of hyoscyamus, one half to four drams, should then be given to overcome the spasm.

\section{Paralysis of the Sphincter Vesicæ}

The condition is often associated with chronic cystitis, continued overdistention causing weakening of the bladder walls and spasm of the sphincter. Spinal diseases, general debility, and old age are further causes.

Symptoms.-Constant dribbling of urine, while upon examination the bladder is found to be empty, is evidence of paralysis of the sphincter. 
Treatment.-This must be directed to correcting the primary condition. If the trouble is caused by old age or debility the patient should be given a good nerve tonic, such as the triple arsenates or other good tonics containing strychnin, and every means taken to improve the animal's general condition. Cantharidin, 1-1000 grain, often works wonders in this condition, and should be given a trial. A good combination is one grain each of pulverized cantharides and of pulverized nux vomica, given twice daily in capsule or pill.

\section{Lithiasis}

\section{(Stone in the Bladder)}

This condition is more common in dogs than is generally supposed, since until the calculus has attained a fair size, few symptoms manifest themselves, a slight cystitis often being the only symptom noticed. When, however, the stone becomes large enough to either partially or completely obstruct the neck of the bladder the following symptoms appear:

Symptoms.-The patient becomes very restless, constantly looking round to his hind quarters and whining. He frequently places himself in the position to urinate, but either accomplishes nothing or succeeds in passing only a few drops of urine, which are often followed by blood. The appetite is suspended, the abdomen becomes distended and painful on manipulation, the back is arched, and the patient straddles and staggers in his gait. If not relieved, the bladder either ruptures or the patient dies of uremia and exhaustion. If a catheter is passed it will go only as far as the neck of the bladder and cannot be introduced into that organ, being stopped by the calculus, consequently no urine will be liberated through it. 
In the bitch the stone may be discovered by the catheter striking it; in the dog, rectal examination will make the diagnosis positive.

Treatment.-The treatment is purely surgical and is described on page 213. As cystitis is nearly always associated with vesical calculi the treatment given for that condition should be followed until the calculus is removed surgically and the cystitis overcome.

\section{Retroflexion}

In this condition the bladder is turned backwards toward the perineal region, often causing it to bulge outward (perineal hernia), and sometimes twisted at its neck.

Causes.-Retroflexion of the bladder may be produced by a prolonged distention of the bladder, with consequent attenuation and weakening of its walls. New growths (pressure), prostatitis, tumors of the prostate gland, constipation, and tramma are further causes.

Symptoms.-This condition results in partial or complete retention of urine, with its accompanying symptoms. If twist has taken place the catheter cannot be passed. Rectal examination reveals the malposition of the bladder, the condition of the prostate, and the occurrence of new growths in the immediate neighborhood. If perineal hernia exists the contents of the hernial sac may be ascertained by puncture, and thus the diagnosis positively made.

Treatment.-This is entirely surgical, and is described on page 217.

\section{Tumors}

The new growths of the bladder most commonly met with are papilloma, sarcoma, carcinoma, myxoma, and fibroma. They may occur either outside or inside the bladder wall. 
Symptoms.-In the early stages, hematuria may result from tumors of the bladder, but later there is pain on urination and the usual symptoms of cystitis. In the last stages the patient becomes emaciated and anemic, and the urine fetid and purulent. The presence of the tumor may be diagnosed by the use of the catheter or sound, and by rectal examination.

Treatment.-Treatment consists of suprapubic cystotomy and removal of the new growth. This operation is described on page 213.

In the majority of the cases the prognosis is unfavorable.

\section{Prolapse}

This accident occurs only when the lower wall of the vagina has been ruptured during parturition. The bladder escapes through the rent and may project beyond the vulva. The condition may be distinguished from eversion of the bladder by the fact that the bladder is covered with serous membrane and contains urine or gradually fills up with urine. In inversion, the bladder is turned inside out, the mucous coat being exposed, and the openings of the ureters, from which drops of urine will be discharged, are easily seen. In addition, examination of the vaginal floor reveals the rupture through which the organ has protruded.

If prolapse of the bladder is neglected, inflammation followed by necrotic changes will take place in the bladder walls, rendering the prognosis grave.

Treatment.-Provided the serous coat is little changed, it must be carefully cleaned with antiseptics, emptied of its contents and pushed back into position, and the tear in the vagina sutured: 'The patient should then receive an anodyne to prevent straining. 


\section{Eversion}

This may occur from the same causes that produce prolapse, from which it differs only in that the bladder is inverted upon itself and that it may occur without rupture of the floor of the vagina. It is differentiated from prolapse by the symptoms given under that head. Remedial measures differ somewhat.

Treatment.-In inversion, after thoroughly cleaning the mucous coat the hind quarters of the patient are elevated and the bladder pushed back throug! the uretha with the finger or a smooth rounded article, such as a thermometer or fountain-pen case. The bladder must then be washed out with a twoper-cent solution of tannin, and straining prevented by anodynes-such as H-M-C or morphin. In cases of recurrence, laparotomy may be performed and the bladder sutured to the abdominal walls, thus effectwally preventing further trouble. 


\section{SECTION XI}

\section{DISEASES OF THE PROSTATE, URETHRA AND PREPUCE \\ Urethral Calculi}

ALCULI in the urethra are very common in $\checkmark$ certain districts and cause great pain and distress to the patients afflicted with them. The calculi may lodge anywhere in the urethra, but are more often found at or near the posterior extremity of the os penis. They vary greatly in size.

Symptoms.-The symptoms of urethral calculi are similar to those of cystic calculi. The animal is restless and evinces pain; makes frequent but unsuccessful, or but partially successful, attempts to urinate; there is some discharge of blood; the abdomen becomes enlarged and is painful on manipulation; the bladder is tightly filled and finally ruptures if not relieved or if death from uremi: poisoning does not supervene. The absence of the stone in the bladder and the limited distance that the catheter can be introduced are means of differentiating between cystic calculi and urethrai conditions.

Treatment.-If the bladder is much distended it must be evacuated by suprapubic puncture. A catheter should be passed up the urethra to the calculus, an incision made down to it, and the calculus removed. The wound is best left unsutured and treated as an open wound.

\section{Urethritis}

\section{(Catarrhal Inflammation of the Urethral Mucosa)}

Urethritis is the result of infection which may extend from the bladder, be introduced by a dirty 
catheter, or during coitus come from a female that is suffering from leukorrhea or endometritis. It is also seen as an accompaniment of distemper. True gonnorrheal urethritis is not a canine disease, but the author has seen one case of urethritis in the dog in which the gonococcus was identified beyonil dispute, on microscopic examination. The bacteria usually associated with non-specific urethritis are the pyogenic organisms, staphylococci, micrococci, and streptococci.

Symptoms.-A sticky mucopurulent discharge from the penis, signs of cystic irritation and an inflamed appearance of the prepuce and meatus are tha chief indication of this disease.

Treatment.-Urinary antiseptics must be employe:1 in this condition. Formin in five-grain doses has given the author better results than anything else. Local treatment is also indicated, and consists of syringing the urethra three times daily with an antiseptic, such as a one-per-cent solution of potassium permanganate or a five-per-cent solution of protargol.

\section{Prostatitis}

Inflammation of the prostate gland usually occurs in connection with some other inflammatory condition, such as cystitis, urethritis, cystitic calculi, constipation, or proctitis. The gland frequently suppurates, and the abscess may discharge into the urethra, or into the abdominal cavity and cause peritonitis and even death.

Symptoms.-Great pain is experienced by the afflicted animal when either urine or feces are passed. Retention of the urine and obstinate constipation often result from prostatitis both because of the mechanical obstruction of the enlarged gland and because of the patient delaying the act as long as 
possible to avoid the pain. Upon rectal examination the prostate is found to be enlarged, hot, and painful on palpation.

Treatment.-The patient should receive a laxative, repeated as necessary to keep the feces soft and overcome constipation. Hot rectal injections give relief from the pain and an anodyne suppository introduced into the rectum, after an enema, also gives good results. If pus forms, indicated by fluctuation and a rise of temperature, it must be evacuated through the rectum after the introduction of a speculum.

\section{Hypertrophy of the Prostate}

This condition is seen most frequently in old dogs, and is often the result of prostatitis. The whole gland becomes enlarged, though one side is usually larger than the other. To the touch, the gland is neither hot nor sensitive. It may feel hard or soft, according to whether the enlargement is due to hyperplasia of the fibromuscular tissue or to infiltration of the gland with purulent fluid.

Treatment.-Laxatives should be administered as required to regulate the bowels; tonics should be given and a generous, nutritions diet supplied. For direct action on the gland itself, administer four grains of chromium sulphate twice daily over a considerable period of time.

Castration has a very beneficial effect in many cases, the gland becoming gradually smaller with the lapse of time. However, the chromium-sulphate treatment should be tried first. Potassium iodid administered internally and the injection of iodin into the gland have been tried, but the results are by no means satisfactory. 


\section{Tumors of the Prostate}

Carcinoma, adenoma, and sarcoma are the principal new growths affecting the prostate and during life can be differentiated from hypertrophy of that organ only by the gradual loss of condition and emaciation of the patient.

Treatment is useless, as the surgical removal of the prostate is not possible.

\section{Phimosis}

Phimosis is the contraction of the prepuce over the free end of the penis. It is often congenital, but is sometimes caused by injury to the prepuce, and subsequent inflammatory swelling, that leaves it thickened or contracted.

Treatment.-Remedial measures are necessary only when the constriction interferes with coitus, and is usually attempted only in stud dogs. It consists in enlarging the opening of the prepuce with a probe-pointed bistoury, sufficiently to allow free protrusion of the penis.

\section{Paraphimosis}

Paraphimosis is the opposite condition from phimosis-constriction of the prepuce behind the glans penis. This usually occurs after coitus, the swelling of the glans during the act of copulation making it impossible for the animal to withdraw the penis ints the prepuce.

Treatment.-First of all, treatment consists in trying to reduce the swollen glans penis with cold astringent lotions and pressure exerted on the end of the penis, while at the same time an effort is made to push the prepuce forward over the glans. These means failing, the constricted portion must be divided with a probe-pointed bistoury, as in phimosis. 
Tumors of the Glans, Prepuce, and Vagina

The principal neoplasms occurring of the glans, prepuce, and in the vagina are carcinomas, sarcomas, and granulomas (contagious).

Treatment.-Complete surgical remural of these tumors should be resorted to where possible, either by means of the scalpel or écraseur, the resulting hemorrhage being controlled by pressure, or, if necessary, by thermocautery. 


\section{SECTION XI1}

\section{DISEASES OF THE TESTICLES AND SCROTUM}

\section{Orchitis}

Inflammation of the testicles, except from tramma, is exceptional in the dog. It is usually caused by kicks, blows, or from crushing, though it sometime: occurs during the course of distemper and other contagious diseases.

Symptoms.-The affected testicle is swollen, hot, and painful on manipulation, the scrotum appearing smooth, tense, and glistening. There is generally considerable constitutional disturbances, indicater by a rise of temperature, anorexia, and a quick, full pulse. The patient shows a disinclination to move about much, and when forced to do so walks with a straddling movement of the hind legs.

Treatment.-A brisk purge should be given, followed up by such febrifuges and sedatives as symptoms indicate. Hot fomentation must be applied to the scrotum or, if preferred, antiphlogistin may be applied in a thick layer as hot as the patient can bear it, and covered with absorbent cotton. In -either case the testicles must be supported by a properly applied suspensory until pain and swelliny have subsided. Inunctions, after fomentation, of hot camphorated oil are particularly beneficial and are preferable to belladonna ointment in being nontoxic.

\section{Abscess of the Testicle}

Abscesses sometimes affect the testicles or scrotum. This constitutes essentially a suppurative 
orchitis and may be due to the same causes as orchitis, enumerated above.

They are treated by incision, drainage, and the usual antiseptic measures. In some cases castration is the only satisfactory means of dealing with suppurative conditions of these parts.

\section{Tumors of the Testicles and Scrotum}

The neoplasms commonly affecting the scrotum and testicles are carcinoma and sarcoma. They are best treated by castration, removing the entire scrotum if necessary. 


\section{SECTION XIII}

\section{DISEASES OF THE HEART AND BLOOD VESSELS}

\section{Pericarditis}

T $N$ canine patients in the vast majority of cases 1 pericarditis is idiopathic and not trammatic. It is usually developed in the course of a general infectious disease, such as acute muscular and articular rheumatism, septicemia, pueumonia, or pleurisy. Traumatic causes may be fractured ribs, punctured wounds of the thorax, or foreign bodies such as needles and fish bones.

Symptoms.-The disease passes through two stages, the dry and the moist or exudative stage.

In the first stage the heart's action is palpitating and tumultuous, and there is a high ferer up to 105 degrees Fahrenheit. The pulse is wiry and fast; dyspnea is present. Cardiac dullness extends upward and backward, and friction sounds are present.

In the second stage, after exudation has taken place the heart sounds are diminished and friction sounds absent. The pulse is small, rapid, and irregular, and the jugular pulse is seen. There is great dyspnea, cyanosis, ascites, and marked emaciation and debility.

Treatment.-Absolute rest and quietness must be enforced and an ice pack applied orer the cardiac region. Good results are also obtained by the application of a mustard poultice. The heart's action must be regulated by the internal administration of from ten to twenty-five minims of tincture of Strophanthus every two hours and 1-S00 grain of aconitine 
every fifteen minutes to effect, then every hour to maintain the effect. In the second stage, when the high fever has subsided, give 1-6+ grain of digitalin and 1-100 grain of strychnin three times a day. Continue the aconitin if the temperature rises above 102 degrees Fahrenheit. In addition to the above, five to fifteen grains of potassium iodid three times daily should be given in milk to assist in the absorption and elimination of the exudate. The bowels must be kept active with repeated doses of calomel, and stimulants must be given if necessary. In cases of great effusion of exudate, paracentesis pericardii must be performed early. The operation consists of introducing a fine trocar and cannula, with all aseptic precautions, through the sixth intercostal space, low down near the sternum and into the pericardial sac, and allowing the fluid to drain away.

\section{Acute Endocarditis}

Acute endocarditis, or acute inflammation of the endocardium, is by no means such a rare condition as is generally thought. In the generality of cases it is produced by the entrance of bacteria into the blood stream. It is a sequel to septicemia, pyemia, septic metritis, distemper, and rheumatism.

Symptoms.-There is tempestuous heart action, with a palpitating, irregular, and later diffused beat. The number of heartbeats frequently outnumber the pulse rate, which often varies from 120 to 240 a minute. In addition to its rapidity the pulse is irregular, intermittent, and very weak, often barely perceptible. The heart sounds, at first normal, become blurred, the first often running into the second, so that it is hard to distinguish them. Later, it blowing sound is heard during systole and a prolonged rustling or vibrating during diastole. The temperature rises to 105 or 106 degrees Fahrenheit, 
and great difficulty in breathing and quickened respiration are noticed. Acute endocarditis may lead to death within a few hours, be prolonged for weeks, or merge into the chronic form.

Treatment.-Absolute quietness and the avoidance of all excitement are essential. The heart's action must be regulated by the administration of digitalin and aconitine, and an ice pack or counterirritant applied to the cardiac area. Staphylobacterins have in many cases given good results, and should be given a trial.

\section{Chronic Endocarditis and Valvular Defects}

Chronic endocarditis usually leads to valvular defects, which are inability to close or stenosis. The inability to close, or insufficiency, arises from shrinkage of the valve itself and shortening of its tendinous fibers, this preventing the valve from completely closing the opening which it guards. Insufficiency and stenosis of the orifices usually go hand in hand, the result being certain secondary anatomical changes in the body, as hypertrophy and dilatation of the heart, engorgement of the liver, kidneys, and spleen, dropsy (ascites) of chest and abdomen, anasarca, and emaciation.

Stenosis is produced by the thickening of the valves and endocardium, the edges of the orifice and of the valves sometimes being covered with polypi-like growths or vegetations. Both orifices and valves lose their elasticity and mobility, preventing the normal amount of blood being driven through without increased force.

Symptoms.-The general symptoms of valvular defects are an easily excited activity of the heart with acceleration of the pulse and beat, palpitation, an irregularity of beat and pulse, abnormal sounds 
during diastole and systole, dyspnea upon exertion, vertigo, rapid exhaustion, emaciation, cyanosis of visible mucous membranes, ascites, anasarca, albuminuria, and diminished renal secretion.

Insufficiency of the mitral valve causes a back flow of blood into the left auricle during systole, producing a blowing or hissing sound as the systole is completed. The diastole sound is abnormally pronounced, there is dyspnea, feeble pulse, and engorgement of the venous system.

The characteristic symptoms of mitral stenosis are diastolic after-sounds, loud pulmonary tone, very small pulse, dyspnea.

Insufficiency of the tricuspid causes a regurgitation of the blood from the right ventricle into the right auricle, with sharp systolic after-sounds, cyanosis, ascites, engorgement of the lungs and renous pulse.

Insufficiency of the aortic valves causes a back flow from the aorta into the left rentricle during diastole, producing a whirring diastolic after-sound. The pulse is strong, rapid and jumping-pulsus celer.

Stenosis of the ventricular aortic orifice gives systolic after-sound, slow, feeble pulse, and in extreme cases cerebral anemia.

Insufficicncy of the putmonary valves gives rise to a diastolic after-sound, feeble heart action, and great dyspnea.

Treatment.-Up to a certain point valvular defect: are compensated for by hypertroply of the auricles or ventricles, and in addition to gond feeding and freedom from exertion require no medicinal treatment. In time, however, compensation relaxes and disturbances are set upon the lungs and renous systems. In these cases it is necessary to regulate the action of the heart, reduce the pulse rate, and increase arterial pressure. For this purpose the ideal drug is digitalis; it equalizes the disturbed circula- 
tion, raises arterial pressure, and lessens the frequency of the pulse. The infusion of the leaves, thirty grains of the leaves to eight ounces of water, may be given in doses of one to four drams daily. In dropsical cases a diuretic should also be given, such as acetate of potash, iodid of potash, squill, and so on. Ascitic conditions may be temporarily relieved by puncture; palpitation and excitement by 1-8 to 1-4 grain of morphin, hypodermically injected. In cases of great prostration give stimulants such as strychnin, brucin (for puppies). whisky, ether, or camphor.

\section{Hypertrophy and Dilatation of the Heart}

This affection consists of enlargement of the heart, increased thickness of its muscular walls, and enlargement of its carities in dilatation of the heart. The heart, or portion of it affected (usually the right side), is enlarged, but the walls relaxed and thinner and its cavities distended. Since from a clinical standpoint both hypertrophy proper and dilatation of the heart are so closely allied as to make it almost impossible to differentiate between them, they are here considered together. Dilatation is nearly always secondary to hypertrophy and appear: when the hypertrophic heart begins to fail to meet the increased demands made upon it.

Causes.-Increased physical exertion, which occurs in racing or hunting dogs as the result of continued severe exertion, is a frequent cause of hypertrophy and dilatation of the heart.

Obstacles to circulation in the blood vessels, sucl, as aortic aneurysms, stenosis of the aurta, thrombi, and pressure of neoplasms on the aorta, degenerative processes in the large vessels, are further causes, as well as valvular defects producing compensatory hypertrophy, lung diseases, emphysema of the lungs, 
tuberculosis, pneumonia, pericardial adhesions, and chronic nephritis.

Symptoms.-Extension of the area of cardiac dullness is an evidence of the disease, as well as dyspnea, vertigo, disturbances of the circulation, and tumultuous heart action. The heart. sounds are irregular, the first loud and vibrating, the second weak and sometimes inaudible. The pulse is weak, irregular, and rapid, and there is a venous pulse.

In addition to the cardiac symptoms proper, consequent upon the disturbances of the circulation, secondary symptoms arise. These may be chronic bronchitis, intestinal catarrh, albuminuria, marantic thrombosis of the arteries, general cyanosis, dropsy, and emaciation.

\section{Rupture of the Heart}

The heart may be ruptured by violence or the walls may give way after being weakened by degenerative processes. Death is practically instantaneous if the rupture is large; if small, death may be deferred for a few or several hours. The symptoms are often the uttering of a loud cry, vertigo, trembling, dyspnea, convulsions, and anemia of visible mucous membranes. 


\section{SIECION XIV}

\section{DISEASES OF THE BLOOD AND BLOOD VESSELS}

\section{Aneurysms}

$A$ NEURYSMS are true and false, the former conA sists of a dilatation of the walls of an artery, which is filled with blood, in the latter all the coats - are ruptured but the blood is retained by the surrounding tissues.

Symptoms.-The general symptoms of aneurysms are: The formation of a pulsating tumor; a peculiar bruit heard over the swelling and pressure symptoms, consisting of pain and paralysis from pressure on nerves and absorption of contiguous parts. The aorta is the artery most commonly affected.

Treatment.-There is no effectual treatment, but rest and avoidance of excitement or exertion may defer rupture and death. In rare cases, where only a small artery is affected and the collateral circulation good, the aneurysm should be removed by first ligating the artery above and below and then excising it.

\section{Hematozoa}

The commonest and most important hematozoa in our patients are the Filaria immitis, the Strongylus vasorum, and the Spirotera sanguinolenta.

\section{Filari Immitis}

This parasite is found in localities rich in marshes and surface water. It is supposed, though not definitely proved, that such stagnant waters become infected by the embryos of the parasite by means of 
the mosquito, which sucks them up from an infected animal. An animal drinking this infected water develops the mature form in its blood. The mature forms chiefly inhabit the right heart and pulmonary artery, while the embryos can be detected in the blood drawn from the peripheral circulation during the night, but retire to the deeper blood vessels during the day.

Symptoms.-In a great many cases no symptoms of a general disturbance to health are manifested. Sometimes death is sudden or has been preceded for only an hour or so by labored breathing and convulsions. More frequently, however, for several days before death the patients exhibit dullness, debility, and more or less frequent epileptiform seizures. Epistaxis, lameness, and paralysis are also observed in some cases.

\section{Strongylus Vasorum}

This parasite also inhabits the right heart and pulmonary artery, producing endocarditis, thrombosis, and embolism.

Besides its usual habitat in nests in the mucous membrane of the esophagus and stomach.

\section{Spiroptera Sanguinolenta}

The Spiroptera sanguinolenta also invades the blood vessels, producing arteritis and thrombosis.

Treatment.-In all of the above infestations treatment is unsatisfactory; but turpentine, in doses of half a dram to a dram, given in emulsion with milk or mucilage, has given some good results. Upon a definite diagnosis being made by finding the embryos in the blood an intravenous injection of salvarsan suggests itself as a rational mode of treatment. 


\section{SECTION IV}

\section{DISEASES OF THE NERVOUS SYSTEM Encephalitis}

CLINICALLY, it is almost impossible to differ$\cup$ entiate between inflammation of the brain substance and inflammation of its corerings, since the symptoms are essentially the same, and also because when one is affected the others are also.

It is most convenient to subdivide brain inflammations into the acute and subacute forms. Undar the former are included all rapidly progressing cases, with extreme symptoms of cerebral inflammationthat is, excitement-and under the second, those. which present signs of depression and are slower in their course.

However, it must be remembered that transitions and intermediate stages occur between the two formi and that therefore no strict boundary can be set between then. The following brain diseases may vecur with symptoms of acute encephalitis-hyperemia of the brain, pachymeningitis, leptomeningitis, encephalitis, emboli, and abscesses.

Subacute encephalitis may occur as a sequel to the acute form, from poisoning from food stuffs, and as a result of toxemia during the course of an infectious disease.

Cause.-Exposure to an excessively high temperature, overexertion, parasites, new growths, emboli, thrombosis, skull traumatisms, otitis media, septicemia, and pyemia all cause disease of the encephalon or the meninges, or botl.

Symptoms.-There are generally premonitory signis 
of the disease, such as restlessness, a desire to hide, and timidity. The paticnt objects to being handled, and althongh never aggressive (see rabies, p. 155) will often bite if touched or disturbed. Loss of coördination is an early symptom of encephalitis, the patient staggering about and blundering into things. There is well-marked conjunctival hyperemia, the head feels hot, and the pupils are contracted. Ophthalmoscopic examination of the fundus of the eye reveals a highly congested condition of the optic papilla and blood vessels.

Fever is usually present, the temperature ranging from 104 to 107 degrees Fahrenheit in the acute form, but in the later stages or in the subacute condition the temperature may be normal or even subnormal. The pulse at first is full and bounding; later on it is slow and feeble, but easily affected by outside stimuli. When nearing the fatal termination the pulse becomes extremely rapid and weak.

The respirations are first increased, but later the rate sinks below normal; they are deep and slow, and each inspiration is marked by a snoring noise (stertor).

Vomiting, (central) epileptiform spasms, and involuntary movements are noticed. Later on, the patient becomes paralyzed, lapses into coma, and dies. Death is frequently very rapid, but chronic conditions such as hydrocephalus amaurosis, deafness, and so on may develop.

Care must be taken to differentiate encephalitis from rabies, but the mistake can hardly occur if we remember that in rabies the typical symptoms are aggressiveness, altered voice, and consumption of indigestible substances. In rabies, too, the temperature of the skull is not so intense nor is the hyperemia of the conjunctiva so well marked.

Treatment.-The patient should at once be placer. 
in a cool, dark, but well-ventilated place, and an ice pack placed on the head; or the head may be continuously bathed with cold water or even placed under a running coll-water tap. In the very early stages-that is, when the pulse is full and bounding -venesection is of the greatest benefit, but later on it is to be avoided as positively harmful. The circulation may also be equally well equalized by repeated small doses of aconitin and veratrin. A full purgative should be given, and the bowels kept well relaxed. Great excitement may be controlled either by a hypodermic injection of hyoscin, a rectal injection of chloral hydrate, or the oral administration of potassium bromid. In sub-acute cases, where the depression is great, recourse must be had to stimulants, such as strychnin, camphor, or ether, administered hypodermically.

Later on, if the patient rallies at all, full doses of potassium iodid should be given.

\section{Anemia of the Brain}

In acute cases of anemia of the brain, vertigo and unconsciousness are the premonitory symptoms. In addition, the heart is weak and the pulse small, vomition occurs, the pupils are dilated, and paleness of the optic papilla is revealed upon ophthalmoscopical examination.

Treatment.-Stimulants (given hypodermically) and circulatory equalizers should be administered and counterirritants applied to the head.

\section{Cerebral Hemorrhage}

Apoplexy consists in rupture of a blood vessel in the brain, with effusion of blood and compression or destruction of the brain parenchyma.

Cause.-A cerebral hemorrhage may take place in connection with encephalitis or from any of the 
causes of that condition. In addition, arteriosclerosis, degeneration of the cerebral arteries, and increased blood pressure are frequently responsible for their rupture.

Symptoms.-Indications of cereloral hemorrhage appear suddenly, and they consist of disturbances of the sensorium, vertigo, incoördination and involuntary movements, collapse, and coma. The visible mucous membranes of the head are intensely hyperemic, and bleeding may occur from the mouth and nose. The pulse is very weak, breathing dyspneic, and sometimes involuntary relaxation of the sphincters vesicæe and ani is noticed. The local symptoms, depending upon the location of the effusion, are paralysis of single muscles or groups of muscles (monoplegia) or semilateral paralysis, hemiplegia, paralysis of the optic nerve, paralysis of sensation of one half of the body (hemianesthesia), and paralyzed deglutition.

Monoplegia indicates hemorrhage affecting the motor centers of the cerebral cortex, while hemiplegia is a hemorrhage in the region of the path of conduction up to the pyramid, paralysis of deglutition, and hemorrhage into the medulla.

Treatment.-Any treatment is usually ineffectual, recovery being only partial, more or less paralysis always remaining.

Absolute quietness and freedom from exciting causes are essential. The application of cold packs to the head, free purgation, stimulants, and later potassium iodid in full doses in the endeavor to cause absorption of the effusion are about all that can be done. Any remaining paralysis should be treated by massage, electricity, and the administration of strychnin. 


\section{Cerebrospinal Meningitis}

This is a rare disease in canine patients. It is of undetermined etiology, and, as its name implies, is an inflammation of the meninges of the brain and spinal cord.

Symptoms.-Great depression and stupor, trembling, fever, incoördination, and involuntary movements are indications of cerebrospinal meningitis. The muscles of the neck are hard and prominent and in a state of spasm, and spasms may affect the whole body, finally causing death by exhaustion.

Treatment.-The same treatment as that for encephalitis, with the addition of full doses of H-M-C hypodermically to control the spasms, is indicated.

\section{Myelitis-Meningitis Spinalis}

\section{(Inflammation of the Spinal Cord and Membrane)}

These two diseases are of rare occurrence and cannot be distinguished clinically. Their causes are mostly traumatic, blows, shocks, vertebral fractures, carious vertebra, metastasis during septicemia, pyemia, and distemper.

Symptoms.-(1) Dislurbance of motion. The gait is stiff and staggering, and there is muscular twitching and partial or complete paralysis. The paralyzed limbs are dragged along the ground and are irresponsive to the electric stimulus.

When the cervical portion of the cord is affected the four extremities are affected and sometimes the pupil of the eye contracted; when the lumbar portion is affected, the hind extremities alone are paralyzed.

2. Disturbances of sensation occur either in a hyperesthesia or anesthesia of the cutaneous nerves. The former manifests itself in pain, excitement, and 
excessive tenderness over the spine. The patient cries or screams when handled, and may attempi to bite when approached; the reflexes are accentuated. The latter is recognized by dulled or suspended reflexes and loss of sensation in the skin, which does not respond to any stimuli.

3. Disturbances of the bladder and rectum. At first both feces and urine are retained on account of paralysis of the rectum and detrusor urinæe muscle, while later incontinence of urine and feces takes place.

Treatment.-In the early stages of the disease cold applications should be applied to the spine in the form of ice packs. Later on, smart counterirritation must be maintained with mustard oil. For the paralysis, the induced electrical current should? be applied and strychnin administered internally. Pain is best controlled by H-M-C. The bladder and rectum should be emptied, and due attention given to the patient to prevent the irritation produced from the urine soiling the thighs and belly if incontinence occurs.

\section{Apoplexy}

Hemorrhage into the spinal cord may be produced by traumatism, neoplasms, exostosis of the vertebra, emboli, atheromatous degeneration, or arteriosclerosis of the spinal arteries. Traumatism is by far the most frequent cause, and the lumbar region most often affected.

Symptoms.-The chief symptom is sudden paralysis, due to pressure of the extravasted blood on the cord, and pain on pressure or movement of the spine or lumbar region. The patient drags his hind legs along the ground, and is unable to stand. More rarely, the hemorrhage takes place more anteriorly, and complete paralysis ensues. The bladder and 
rectum are usually affected, as in inflammation of the cord, and the paralyzed numseles do not react to electrical stimuli.

Treatment. - Treatment of apoplexy of the spinal cord is fairly successful, but usually of long duration. In order to promote absorption of the extravasated blood, potassium iodid to saturation should be given and frequent fomentations applied to the spine. The affected muscles should receive electrical massages or friction with stimulating liniments. The bowels must be kept freely active with laxatives and enemas, and every attention given to the comfort and cleanliness of the helpless patient.

\section{Epilepsy}

This is a chronic brain affection, with fits of disturbed consciousness and sensation and accompany ing muscular spasms, between which are varying intervals of freedom from symptoms. The condition is probably due to some fleeting disturbance in the cells of the cerebral cortex and motor cortices centers. Its essential causes are not known, but there is ample evidence to establish the fact that it is undoubtedly hereditary. In individuals predisposed to the disease sometimes an attack is brought on by great excitement, sudden fright, or overexertion. It is a comparatively rare disease in our patients, although they frequently suffer from epileptiform fits, which, however, can generally be accounted for by injuries to the skull which produce concussion of the brain either by depressed fractures or blood clots, and give rise to epileptiform seizures or fits. Fits are also caused by reflex action, painful wounds of the extremities, or body, irritation of the alimentary mucous membrane by parasites, constipation, and by foreign bodies in the alimentary tract. Overexertion during excessively hot 
weather, with deprivation of water, is another very common cause.

Symptoms.-The patient, after staggering around for a moment or so, suddenly, falls to the ground in convulsions, uttering loud cries, champing the jaws, and frothing at the mouth. The animal struggles convulsively with its feet, goes into convulsions, and loses conscionsnes. In some cases the patient seems to have an irresistible desire to run, and tears along, foaming at the mouth and champing. the jaws, finally to fall and go into convulsions before losing consciousness. These cases generally give rise to the cry of "mad dog," the unfortunate only. too often being mercilessly clubbed to death or mangled by ineffective shots from revolvers of zealous but incompetent guardians of the law. During the fit, breathing is rapid and dyspneic, the pupils dilated and insensible to light, reflexes suppressed. and the pulse slow, small, and hard. The convulsions sometimes cease suddenly, at other times gradually, and often give place to general tetanic spasms, during which the involuntary passage of feces and urine takes place. When the attack is over the patient usually quickly regains consciousness, but remains exhausted and dazed for some time. But sometimes the attack is ended by a deep, snoring sleep. In many cases the animal is extremely irritable and until completely restored to its normal mental equilibrium shows a tendency to bite, if handled. Fits may last from fifteen seconds to half an hour and recur quite frequently, or the periods of intermission may be quite prolonged.

Treatment.-During an attack very little can be done, with the exception of preventing the animal from knocking itself about, wedging open the jaws to prevent laceration of the tongue, and applying cold water or ice to the head. Afterwards all discoverable 
causes, such as worms, constipation, and so on, must be removed, and in the case of depressed fractures of the cranium surgical means must be employed, if possible, and the patient placed on a course of potassium iodid and solanin (one grain) three times a day, or one grain of silver nitrate daily in three doses. The bromids so long used in medicine for the relief of epilepsy do not produce what is claimed for them, and certainly are ineffective.

\section{Chorea}

This troublesome nervous infection is discussed under distemper (see p. 151). 


\section{SECTION X VI}

\section{DISEASES OF THE EYE \\ Conjunctivitis}

T NFAMMATION of the conjunctival mucous 1 membrane may vary in its intensity from a simple hyperemia to a purulent condition. It is caused by foreign bodies-such as small particles of dirt, ashes, or metal-by smoke and irritant chemical fumes, and by too strong antiseptic washes. It is a very frequent sequel to an antiseptic bath given for the purpose of getting rid of parasites or even from the use of cheap and crude dog soap. Direci injury may sometimes be the cause of conjunctivitis, and it is often seen as a symptom of some other condition.

Symptoms. - The first symptom usually noticed in an acute attack of conjunctivitis is a flow of tears from the affected eye, which is nearly or completely closed. There is great intolerance to light (photophobia) and upon examination the conjunctiva is found to be congested (hyperemia) and swollen. If the case becomes of the purulent variety the watery discharge (tears) becomes pus-like and the bulbar conjunctiva may become involved, as evidenced by marked pericorneal injection and edema around a slightly hazed cornea. In severe cases corneal ulcers form from erosion of the epithelium. The eyelids often become glued together by the discharge, which excoriates their edges and oftentimes the skin of the cheeks.

Treatment.-The affected eye should be bathed with cold water and then carefully examined for 
foreign bodies, which should be removed at once if detected. If the eye be very sensitive, a few drop. of a four-per-cent solution of cocain instilled into the conjunctival sac greatly facilitates the required manipulation. The eye should then be washed with a saturated solution of boric acid, a 1-3000 chinosol solution, or a two-grain to the ounce solution of protargol. If there is much pericorneal injection and haziness of the cornea, the pupil should be dilated with homatropin. Excessive pain and irritation are nicely controlled by a few drops of a collyrium containing one grain of cocain and ten grains of boric acid to the ounce of water. When the acuie stage is passed the eye should be frequently cleansed with the saturated solution of boric acid. and a few drops of a solution of protargol, five grains to the ounce of distilled water, instilled three or four times a day. Cloudiness of the cornea should be treated, after the subsidence of all acute symptoms, with the yellow oxid of mercury ointment, one to three per cent.

\section{Keratitis}

Inflammation of the cornea may be the result of infection spreading from an infected conjunctiva or introduced by injuries, and it is a frequent symptom of distemper. Keratitis is classified as heratitis superficiatis, keratitis interstitiatis, and suppurative keratitis.

\section{Keratitis Superficialis}

Keratitis superficialis should in reality be regarded as a disease of the conjunctiva, since it is the bulbar portion of that membrane which is affected, and, strictly speaking, not the cornea proper. Consequently it has been described above under conjunctivitis. 


\section{Keratitis Interstitialis}

Keratitis interstitialis is a far more serions condition, since it involves the corneal parenchyma.

Symptoms.-It begins with irregularly rounded whitish dots which usually appear in the center of the membrane. There is haziness, ciliary infection, and a marked formation of new vessels. At times the opacity is so completely confined to the interstitial lamina that the cloudy portion of the membrane looks as if it were covered. As the diseas? progresses the opacities in the lamellae grow larger by fusion until the whole cornea may become opaque. There is intense ciliary injection, a constant flow of tears, and great photophobia. The amount of vascularization varies largely in different cases. Vessels extend in from the superficial and deep loops, until at times the entire membrane becomes of a dark red tint. Their formation generally occurs in that part of the periphery that is nearest the densest opacity. When the disease subsides the corne: clears from its periphery, the central opacities being the last to disappear. The complications are iritis, with the formation of posterior synechia (adhesion of iris to lens), choroidal degeneration, and retinitis.

\section{Suppurative Keratitis}

\section{(Abscess of the Cornea)}

Suppurative keratitis in its various forms is due to injury and infection or is secondary to disease affecting other tissues of the eye or body.

The most common form is marked by ulceration of the cornea, the ulcer sometimes destroying all the layers and causing evacuation of the aqueous humor. Usually, however, the ulcerative process stops at the membrane of Descemet, which from interocular pressure bulges out from the cornea, causing the condition known as staphyloma. 
Abscess of the cornea is an exceedingly serious condition, the eye nearly always being lost. It generally results from injury, with subsequent infection by pyogenic organisms. (In such cases we fincl near the center of the cornea a yellowish-gray rounded spot, infiltrated with pus cells, with an area of swollen tissue around it.) Later the anterior walls slough off, and an open ulcer with prominent edges is revealed. Soon cloudiness of the aqueous humor appears and pus forms in the anterior chamber of the eye (hypopyon).

The infective processes extend to the iris and ciliary body and finally the whole eye becomes involved, there being no tendency to spontaneous recovery.

As all varieties of suppurative keratitis are serious and threaten the eyesight, treatment should be at once instituted and diligently carried out.

Treatment.-The eye should be frequently irrigated with antiseptic solutions such as chinosol (1-2000), a saturated solution of boric acid, or bichlorid of mercury (1-5000).

The pupil should be alternately dilated and contracted by the instillation of solutions of atropin (1-100) and physostigmin (1-200) to guard against adhesions of the iris.

The ulcers themselves should be touched up with a solution of protargol, five grains to the ounce, or, if sluggish, even by thermocautery under cocain anesthesia.

Very small staphylomas should be punctured; by diminishing the intraocular pressure, healing is accelerated. Partial staphylomas should be ligated with a fine silk ligature and ablated three days after ligation.

Abscesses of the cornea should be opened without 
delay with a Graefe's knife after the manner of Soemisch. This method consists in introducing the knife in the sound corneal tissue on one side of the abscess, the back of the instrument being held toward the center of the eye and its edge turned forward. Maintaining this position, the abscess is bisected by bringing the knife out on the opposite side of the cornea, the cut being made horizontally. By this method the ulcerative process is checked and any pus in the anterior chamber evacuated. It must be remembered that opening the anterior chamber is an essential part of the operation, and responsible for its success.

\section{Opacities of the Cornea}

Corneal opacities are sometimes congenital, but generally they are the result of inflammation, injury, or ulceration. According as they present a slight haze, a defined spot, or a dense and permanent opacity, they are termed nebulæ, maculæ, or leukomata. As the tissue of the cornea is resistant and inelastic, any loss of substance is made up by exudation and subsequent organization of connective tissue and epithelium; consequently the opacity is as large as the original loss of substance. Vision is affected not only according to their extent, density, and position, but by reason of the alteration which they cause in the corneal curvature.

It is remarkable what great reparative powers the corneal tissues have and to what extent the effused lymph will clear. Consequently in growing animals, with perhaps the exception of the scar left by an abscess or deep ulceration, the prognosis of good eyesight with any small opaque scar is good. With older animals, however, owing to their low power of recuperation the prognosis is the reverse. 


\section{Congenital Opacities of the Cornea}

Congenital opacities in many cases clear up or diminish a few months after birth, others again persist, and may cause total blindness.

Treatment.-The eye should be left alone until all active inflammation has subsided, since the rationale of all treatment is stimulation.

Solutions of protargol, five grains to the ounce, or six-per-cent ammonium chlorid give excellent results when instilled into the conjunctival sac three or four times a day, but probably the best agent for this condition is three-per-cent yellow oxid of mercury ointment, a small quantity of about the size of the head of a match being introduced twice daily.

In persistent cases intramuscular injections of fibrolysin should be tried, as brilliant results are sometimes obtained from its use.

\section{Iritis}

Inflammation of the iris is characterized by severe pain, especially when the eye is exposed to strong light, sluggishness of the pupil, pericorneal injection, and change in color from the normal to yellowishred or to red. Synechix are nearly always its sequela, and sometimes in cases of profuse exudation there is exclusion of the pupil.

Treatment.-If the eye is protected from light and irritants, mild cases of iritis will recover with local treatment by atropin, adrenalin, and cocain In more severe cases, however, the local treatment should be supplemented with corneal puncture (to relieve tension) and free purgation (calomel). In all cases full dilation of the pupil must be secured to guard against or to break down already existing synechice. Hot compresses over the affected eye and a full dose of H-M-C are of great assistance 
in controlling the severe pains associated with this condition.

\section{Cataract}

\section{(Opacity of the Crystalline Lens)}

The word "cataract" is applied to any opacity or want of transparency of the lens, whether partial or complete. It may be congenital or it may occur at any time in the animal's life. It is often produced by wounds of the lens or its capsule, or it may be caused by any disease of the eye that seriously interferes with the nutrition of the lens. It is a disease common to old age, being then caused by im. paired nutrition.

Treatment.-Only recourse to surgery is of any avail, and in dogs this gives fairly good results, although theoretically it is useless.

The most practical and successful operation is that of discission, which consists of making a crucial incision into the anterior capsule of the lens, thus allowing access of the aqueous humor, which first of all causes swelling of the lens, then its absorption.

The technic is as follows: The patient is anesthetized, the conjunctival sac washed out with a 1-5000 chinosol solution and then with sterile water. and the pupil dilated to its full extent with atropin (if $\mathrm{H}-\mathrm{M}-\mathrm{C}$ is used as the anesthetic, this will be unnecessary). The eyelids are then held apart with an eye speculum, and the discission needle introduced through the cornea at its lower median periphery. When the needle reaches the anterior causule it is drawn across it twice in a crucial manner and slowly and gently withdrawn. Mbsorption takes place in about three months. The after-treatment consists in lieeping the patient in a darkened kennel 
until all signs of irritation have subsided, and in using mild antiseptic collyria.

\section{Diseases of the Retina}

Diseases of the retina are not common in dogs, the two diseases of this nature most frequently met with being detachment of the retina and hemorrhage, both being generally the results of traumatisms. Beyond allaying pain and irritation very little can be done in the way of treatment, blindness always being the result in the first instance, and generally in the second.

\section{Glaucoma}

This name is given to a group of eye symptoms characterized by periodical obscurations of vision in association with an increase of intraocular tension, haziness of the cornea, dilatation of the pupil, pericorneal venous injection, and a dull purplish discoloration situated around the margin of the cornea. At times this condition may be accompanied by agonizing pain; at others no discomfort is manifested, but there is a gradual loss of vision.

When the disease has recurred frequently, excavation of the intraocular end of the optic nerve takes place and atrophy of the entire eyeball ensues.

There is often total or partial anesthesia of the cornea and a characteristic rigidity of the whole eye to the touch.

Treatment.-Treatment is unsatisfactory, but may delay or even inhibit the progress of the disease.

Twenty-four hours previous to operation the pupil should be widely dilated with eserin frequently instilled into the eye.

Operation.-With the patient under a general anesthetic and with the usual precautions as to asepsis, a narrow knife is cntered at the cornea- 
scleral junction and thrust out at a similar point on. the other side of the anterior chamber. Care must be taken to prevent prolapse of the iris, but should this accident occur, the prolapsed portion should be removed with scissors and the remainder gently replaced. The instillation of eserin will tend to prevent the prolapse and should be used freely both before and after the operation.

\section{Ailments Amenable to Surgery Alone}

Injuries, foreign bodies, enucleation and other eye troubles will be discussed under surgery of the eye in Section III (see pp. 194-202). 


\section{SECTION X VII}

\section{DISEASES OF THE EAR}

THE ears, by reason of their exposed position on 1 the body, receive injuries as a result of accidents. These may vary from slight wounds to serious lacerations, resulting from fighting, becoming entangled in barbed wire, or in running through underbrush while hunting. The wounds thus produced require treatment on general surgical principles or special operative interference such as will be described under plastic surgery of the ear (p. 189). Contusions, if severe, produce hematoma, which will also be dealt with in a later section (see p. 188).

The ears are also often affected in the various. skin diseases, both parasitic and non-parasitic, from which our patients suffer.

\section{Ulceration of the Earflap}

This condition is usually the result of infected wounds, the parts becoming infected either by the patient's claws, by flies, or by the discharge associated with catarrh of the auditory passares. It produces great irritation, which the patient $\mathrm{tr}^{\circ}$ es to relieve by scratching and shaking the head, which maneuvers, however, only tend to make matter:3 worse.

Treatment.-First of all, thoroughly cleanse the parts, using the curette if necessary, and paint these sores with tincture of iodin. The ear sluvild then be inclosed in antiseptic gauze and an ear cap, net, or bandage applied. The patient must at all costs be prevented, either by hobbling or by the use of 
the Elizabethan collar, from scratching or rubbing the parts.

\section{Catarrh of the Auditory Canal}

\section{(Canker)}

Causes.-Dust, dirt, and water getting into the ear, either during swimming or while the dog is being bathed, and the ear not being afterwards properly dried, are a frequent cause of catarrh of the auditory canal. Parasites, the symbiotes auricularis var. canis, are a not uncommon cause of the trouble.

Symptoms.-The patient shows great irritation by scratching, rubbing, and holding the head with the affected ear downward, and pain is manifested by the cries and yelps which accompany any manipulation of the ear. Upon closer examination, caked around the external auditory meatus and matting the hair surrounding it, a brownish, sticky discharge with a characteristic odor is noticed. If the base of the ear is gently pressed a sucking sound is heard and the patient evinces pain. In parasitic canker the parasites may be discovered by a careful examination with a magnifying glass. In exceptionally severe and long-standing cases the middle and internal ears may become involved, deafness and sometimes fatal meningitis being the results.

Treatment.-The patient should be secured on the operating table in the abdominal position and muzzled, to prevent accidents. The ear should then be thoroughly but gently cleaned with hydrogen peroxid and absorbent cotton swabs. It is essential that the whole of the external ear, comprising ear flap, external auditory meatus, and auditory canal, be cleared of every vestige of discharge at the first treatment. The ear and canal should then be thoroughly dried and the canal filled up with either of the following dusting powders: Chinosol (fiftcen grains), or for- 
midin or aristol, or bismuth formic iodid, and boric acid (one ounce).

An ear cap or bandage should then be applied to prevent the patient flapping his ears, and means should be taken to prevent scratching of the car. With some very sensitive animals it is a good policy to kecp them under the influence of H-M-C until the irritation has subsided, otherwise it may be impossible to prevent the patient aggravating the trouble by his continual efforts to scratch, rub, or flap the ears.

After the first treatment, all subsequent dressings should be in powder form, washing the ear or passage or the use of lotions or liquids of any description being only productive of pain. It is surprising how even the most obstinate cases of canker yield to dry dressing after resisting even the most conscientious treatment with fluid antiseptics.

In the parasitic form, after cleansing the ear as before described, the most effectual parasiticide is one dram unguentum hydrargyri nitratis and one ounce oil of almonds, the ear being smeared with the ointment and the canal filled with it, and the meatus plugged with cotton wool. The ear cap is applied as in an ordinary case and the ear cleaned up again on the third day, after which the treat. ment consists of dry dressings as in simple canker.

\section{Deafness}

An inability to hear may be either congenital or acquired. The former is incurable, and need not be considered further. Acquired deafness may be the result of an undue accumulation of wax, catarrh of the external auditory canal, ulceration of the tympanum, abscess and destruction of the middle ear, new growths, or paralysis of the auditory nerve.

The only cases that are at all amenable to treat- 
ment are those resulting from accumulations of wax, some forms of neoplasms (polypi), and canker. Paralysis of the auditory nerve occasionally yields to the influence of strychnin and a prolonged course of tonics.

Accumulations of wax are best treated by first of all filling the external auditory canal with hydrogen peroxid, which loosens up the deposit, and then removing the wax with forceps, swabs, or an ear curette. Ulcers should be first curetted and then touched up with a stick of nitrate of silver. Polypi are to be ablated either by excision with scissors and scalpel or by the use of a small wire écraseur or snare. 


\section{SECTION XVIII}

\section{DISEASES OF THE SKIN}

$\mathrm{F}^{\mathrm{ROM}}$ a clinical point of view the diseases of $F$ the skin may be considered under two headings -parasitic and non-parasitic.

Parasitic diseases of the skin are produced by animal and vegetable parasites, while non-parasitic diseases of the skin are idiopathic or exanthematous.

\section{Sarcoptic Mange}

This is a parasitic skin disease caused by the burrowing into the skin of the Sarcoptes squamiferous and is characterized by inflammation, pruritus, and loss of hair. The eruption at first resembles flea bites, but the patient's efforts to relieve the irritation by scratching may cause the whole area to become erythematous. Papules then make their appearance, later becoming vesicles that eventually burst, leaving little raw spots. The usual sites of this eruption are the head, bridge of the nose, base of the ears, eyebrows, the belly, around the elbows, root of tail, the paws, the inside of the legs, and sometimes the genitals. It spreads rapidly and may involve the whole body in less than a month. In neglecterl cases the skin becomes greatly thickened, wrinkled, covered with scales and scabs, and ofter traversed by fissures. Warmth and exercise increase the itching. It is very contagious, and patients should be rigidly isolated.

Treatment.-Isolate the patient and clip off the hair from the whole body. Wash thoroughly in warm water and green soap to remove dirt, scab, 
and so on, then dip in the following solution, brushing it in thoroughly with an old hairbrush:

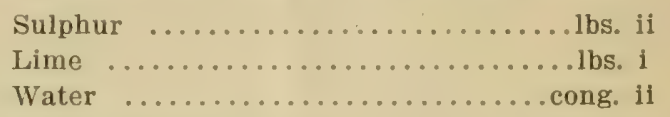

Boil this mixture until it turns a deep orange color and make up to five quarts, with water. Repeat the treatment in ten days. In mild cases it is sufficient to clip the hair around the affected spot and apply the above solution, or in sensitive situations like the eyelids or eyebrows, equal parts of balsam of Peru and olive oil make a most satisfactory substitute. Repeat the treatment every ten days until a cure is effected.

\section{Follicular Mange}

Follicular mange is one of the most serious skin diseases from which our patients suffer, the parasite being so deeply situated that parasiticides have no permanent effect and bacterin treatment is effective in only a few cases.

Cause.-Follicular mange is caused by a parasite, the Demodex folliculorum, which gains access to the sebaceous glands and hair follicles and there multiplies. These parasites produce the characteristic symptoms of the disease by the irritation they themselves create and by opening the doors for the invasion of pyogenic organisms.

Symptoms. - The disease exists in two forms, the squamous and the pustular. In the former the regions affected are usually around the eyes, lips, throat, breast, and legs, but it may extend to the whole body. The skin becomes inflamed and covered with scabs and the hair falls ont, leaving bald patches. By gently scraping the parts with a blunt knife and placing the debris so collected under the microscope the parasite can be casily discovered. 
This parasite is easily distinguished from that of sarcoptic mange in that the latter is shaped somewhat like a horseshoe, while the former is elongated, has four pairs of legs and a segmented abdomen, and in shape somewhat resembles an oat grain. (For full description, see works on parasitology.)

In the pustular form, pustules varying in size from a millet seed to the size of a pea are found studded all over the affected area. These pustules sometimes become confluent and larger areas of skin become necrotic. On pressure of one of these pustules, a sanguineous pus exudes which, if examined under the microscope, reveals the presence of numerons demodecidre. As time goes on the skin becomes covered with scales and much thickened. Fissures and cracks make their appearance, become infected, and exude a bloody pus that cakés on the skin and gives the patient a most loathsome appearance. Irritation as a rule is not great, and sometimes even entirely absent, but the skin is tender, and scratching or rubbing the affected parts is resented by the patient. In addition to the visible lesions there is a characteristic monsy odor emanated from the patient that, once experienced, is unmistakable.

Treatment.-This is most unsatisfactory, the usual parasiticides being practically useless. The only method at present that holds out any hope of permanent relief is the bacterin treatment.

The patient must first of all be thoroughly washed with green soap and a mild coal-tar antiseptic to remove all accumulations of scales and discharge. Evacuate all pustules and give another antiseptic bath (kerosene emulsion is suitable).

Every three to five days inject hypodermically it staphylobacterin. Dust the whole body with prepared chalk (creta preparata) often enough to keep 
the affected parts well covered. . Keep evacuated all pustules that form, but use no further baths or antiseptic washes. If the treatment is successful and pustule formation ceases, the remaining dermatitis can be relieved by inunction of Lassar's paste,* or of a paste composed of :

Ac. salicylic ............... 2 parts

Amylum .................24 parts

Zinc oxid ...................... parts

Petrolatum alba ...........50 parts

Ringworm and Favus

Both of these ailments are caused by vegetable parasites of the genus hypomycetes or fungi.

Favus is caused by the Achorion schönleinii, which, by attacking the skin and hair, causes destruction of the latter and the formation of cuplike depressions and scab formation in the former. These scabs (scutula) are of a roundish shape, concave in the center, and of a yellowish color. When removed they leave a depression in the skin which is thin and discolored with blood. The hair in the affected portion of the skin falls out, leaving bare spots, and the irritation is considerable.

Ringworm is caused by another fungus-the Trichophyton tonsurans-which invades the hair with its mycelium, the spores as a rule being clumped round outside the hair. This invasion of the hair and its root produces inflammation of the follicle; and renders the hair brittle, so that it breaks off. The clinical symptoms of ringworm are varying irritation, circular bald patches on the skin, slightly elevated at their circumferences and covered with dry, grayish-colored scabs. Upon close examination a few stumps of hairs which have broken off are observed in these bald patches. If an affected

\footnotetext{
*Lassar's paste consists of vaselin six ounces; zinc oxide and starch of each six drams and salicylic acid thirty grains.
} 
hair be pulled out with the forceps a white-gray fuzz is observed around its roots. If placed upon a slide, treated with a drop of potassium hydroxid, and placed under the microscope the spores can be seen ranged around or in the hair and the felt-work of the mycelium penetrating its substances.

Both favus and ringworm are contagious to man and other animals, and every care should be used to avoid the spread of these diseases.

Treatment.-Practically the same treatment is given for both conditions. In farus all loose hairs should be removed with the forceps and the scutula lifted off so as to expose the underlying skin. In ringworm all affected hairs must be pulled out and the healthy hair clipped away around the circumference of the affected area, as the disease spreads from the periphery. In both favus and ringworm, tincture of iodin should then be painted both on the affected portions of the skin and that immediately surrounding them. Treatment is often rather tedious, fresh patches developing as one is cured. Sometimes, for some unexplained reason, iodin seems to be ineffectual to promote a cure, and in these cases the author has had most excellent results from giving the parts an application of bichlorid of mercury, 1-300, in alcohol. When the parts are dry follow with an ointment as follows:
Ac. Salicylic
Ac. benzoic, aa.................... $\mathrm{xv}$
Petrolatum alba ................... i

Care should be exercised to burn all extracted hairs and scabs and to disinfect the hands after applying treatment in order to prevent infection. Patients should, of course, be isolated, and the kennels disinfected and bedding burned before other patients occupy them. 


\section{Parasitic Dermatitis}

The dog flea, Pulex serraticeps; the dog louse, Trichodectes latus; ticks, Ixodes, and harvest bugs, Trombidii, often extensively infest our patients and are the cause of much irritation and a mild form of dermatitis, which is augmented by the patient's efforts to relieve the itching by scratching. If the superficial abrasions caused by the animal's claw become infected, very troublesome and sometimes extensive sores are produced. Both fleas and lice should be eliminated as quickly as possible, since they are not only a drain on the patient's constitution but may be the intermediate hosts of the Taenia canina.

Fleas are fairly easily gotten rid of by a thorough washing in a creolin solution. It must be remembered, however, that the female flea does not lay her eggs on the animal, but in cracks and crevices of the floor and sides of the kennel, and in bedding, dust, and filth, so that in addition to the treatment of the patient, his bedding, kennel, or sleeping place must also be thoroughly cleaned either with boiling water or a strong solution of creolin in order to destroy the eggs and larve. If this latter precaution is omitted the patient will be reinfested in a very short time.

Lice are much harder to eradicate than fleas, for although the adults are easily enough disposed of by bathing in creolin water or by applications of tincture of larkspur, yet the "nits," or eggs, which are attached to the hairs, are extremely resistant to the action of parasiticides on account of their keratinous covering. The patient should first of all be washed in a strong solution of sodium bicarbonate, which softens the nits, allowing the subsequent application of the chosen parasiticide to destroy the 
contained embryos. In addition, as many nits as possible should be removed by the use of a finetoothed comb.

Ticl's infest the dog and cause considerable irritation and obstinate sores if the invader is pulled off, as it invariably leaves its pinchers behind. The most effectual way to relieve the patient from ticks is to drop a few drops of gasoline onto each individual tick, which immediately lets go and can then be removed and destroyed. A ready way to remove ticks while camping or in the woods where gasoline is not available, is to make the tick let go by holding the end of a lighted cigarette or cigar to its posterior extremity.

Iharvest bugs also infest dogs, and are the source of much irritation. They yield to the lime and sulphur dip or to application of benzine or gasoline.

The dermatitis resulting from the invasion of any of the above vermin is easily controlled by the application of mild antiseptic lotions or Lassar's paste. Infected sores should be disinfected with tincture of iodin and dressed with an antiseptic dusting powder.

\section{Erythema}

This disease consists of a hyperemia of the papille and superficial layers of the skin.

Cause.-Erythema occurs either indepenciently or forms the introductory stage of other skin diseases. Its causes are mechanical, due to pressure, whipping (wheals), dipping dust, and friction; the result of chemical irritants, due to washing with crude soap, unduly strong antiseptics, insect bites, and decomposition of urine on the skin near the place of discharge during paralysis of the hind legs; or thermal, the result of excessively high or low temperatures, hurns and scalds of the first degree, or sunburn. 
Symptoms.-More or less redness, diffuse or localized, which can be made to disappear temporarily by pressure of the finger, is an indication of erythema. This redness is often accompanied by intense pruritus.

Treatment.-The cause, if possible, should be removed and the affected parts soothed with applications of liquor plumbi subacetatis, Lassar's paste, or oxid of zinc ointment. Burns and scalds should be dressed with a saturated aqueous solution of picric acid, and intense itching may speedily be relieved by painting on a six-per-cent solution of silver nitrate.

\section{Eczema}

Eczema is a dermatitis, manifesting itself in various stages of development and intensity. The stages which a typical eczema goes through are as follows, but it must be remembered that the disease may terminate or become chronic in either one of them, also that the clinical picture may be quite materially altered in appearance by the patient's rubbing, scratching, or biting to relieve the pruritis.

1. The erythematous stage, which consists of hyperemia of the skin, with slight exudation and increased proliferation of the epidermis, which later becomes exfoliated as scales or scabs.

2. The papular stage, characterized by the formation of small red papules, which are caused by smallcelled infiltration and serous saturation of the individual papilla of the dermis.

3. The vesicular stage, which develops when the serous saturation is so extensive as to rupture the cells of the rete mucnsum, the fluid then penetrating as near to the surtace as the stratum corneum.

4. The moist or weeping stage, which results 
from the rupture of the vesicles either spontaneously or from traumatic causes.

5. The pustular stage, which results from the transformation into pus of vesicles filled with serum. These pustules often become confluent, producing large suppurating areas.

6. The scaly stage, which results from the drying up of the exuded matter and the desquamation of the dead epidermal cells.

As before stated, it is by no means necessary that the disease pass through all the above stages. An early stage may pass directly into the last, or the ecrema may long remain persistently in the same stage.

Causes.-Eczema is usually set up as the direct result of some external irritant acting upon the skin, such as dust, mud, fleas, lice, too frequent bathing, and the use of soap. Injurious foods and digestive disorders with faulty elimination no doubt act as predisposing causes, but the disease must be attributed to direct local irritation.

Symptoms.-In the initial stage, which very often passes unobserved, bright red spots appear, surrounded by a hyperemic area. These spots rapidly run together, the affected skin becoming spongy, turgescent, and of a high temperature. The hair in the affected area stands erect, and the skin is tender and irritable. Owing to the patient's efforts to relieve the pruritis, the pustular and scaly stage may immediately be entered upon, but if the disease rums its regular course, small blisters develop from the pimples. By confluence of these vesicles moist and reddened areas of skin are formed from which the hair falls out. These areas are covered with a serous, sero-fibrinous, or even purulent exudate, and are exceedingly sensitive to the touch and show a tendency to spread. 
Frequently the vesicles change into pustules, pustular eczema then being the result. The blisters enlarge and their contents grow dull and become purulent. The pustule may remain isolated, spread in groups, or run together, producing patches of various size. The hairs then become erect, stick to each other, and are glued together by the exudate into stiff tufts, which become felted and are easily pulled out or fall out spontaneously. The skin thus laid bare is found to be thickened, exquisitely tender, and covered with a yellowish, creamy, sticky pus.

Healing follows by the drying up of the purulent discharge into scabs and crusts, under which suppuration persists for some time. Similar localized purulent inflammations of the skin occur, without any preceding stages, as a direct result of mechanical, chemical, or thermal irritation.

Eczema may in all its stages become chronic. The most important factor here is to prevent the patient from rubbing the affected areas.

The changes produced in the skin by chronic eczema consist in persistent hyperemia, higher temperature, and thickening. The skin gradually becomes hard and dry and loses its elasticity. The hairs become thin and stray (compression and atrophy of the hair papille and their blond supply).

The condition of the patient in ordinary cases remains unaltered except for restlessness and often extreme thirst. In cases of long duration, however, the continued irritation in time produces emaciation and, in weakly subjects, results in cachexia and death. Acute eczema may last for from one to three weeks, while the chronic form of the disease persists for months or years and is frequently incurable.

Treatment. The treatment varies both according to stage and individuality. The main obstacle to 
success is the persistent itching and the patient's efforts to relieve it. If possible, the cause must be removed, and in all cases the alimentary tract should be cleaned out by a brisk saline purgative and kept clean by the daily administration of five grains of the sulphocarbolates of zinc, calcium, and sodium in water three times a day. Contrary to popular opinion, diet exercises little influence either on the production or course of the disease, but often a complete change of diet gives wonderful results. Bathing and the use of soaps are absolutely to be avoided during an attack and used only with great discretion upon recovery, or a relapse mù occur.

In the earlier stages of eczema erythematous, papular, and vesicular, soothing and emollient applications are to be employed. Liquor plumbi subacetatis applied to the affected parts, followed by a dressing of zinc oxid ointment or Lassar's paste, is most efficient.

In the later stages (weeping or pustular) the matted hairs should be clipped off and the parts cleaned with hydrogen peroxid and dried with absorbent cotton swabs. The affected parts should then be painted with a six-per-cent aqueous solution of silver nitrate and kept dusted with a desiccant dusting powder such as ten parts of aristol, formidin, or boric acid, ten parts of starch, and one part of tannic acid.

For chronic eczema a more stimulant line of treatment is necessary. The hair should first all be clipped off and the skin dressed with any of the following dressings which are all effective, although the first mentioned is most excellent: Four parts of oil of tar, forty parts olive oil, one part iodin glycerin; thirty grains chrysarobin to one onnce vaselin, or sulphur ointment B. P. 
Internally, administer five to ten drops of Fowler's solution once à day.

\section{Urticaria}

Urticaria is manifested by sharply defined, flat, raised swellings of the skin, the result of quickly occurring serous transudation in the papilla of the dermis. The cause of this transudation of fluid is the sudden dilatation of the capillaries, consequent on undue stimulation of the vasomotor nerves.

This affection of the vasomotor nerves may be due to external irritation or it may be of internal origin. The external irritants may be bites of insects, stinging nettles (Urtica urens), or the hairs of caterpillars.

The internal causes of urticaria are due to certain kinds of foods which, owing to individual susceptibility, act as toxines, or to the absorption into the blood stream of toxic matters from the alimentary canal (fecal toxemia).

The pomphi are sudden in their appearance and frequently run together, producing large edematous areas. The treatment consists in warm fomentations and the administration of a smart saline purge.

\section{Alopecia}

Falling out of the hair occurs as an independent condition and may affect the whole body (alopecia celsi), or it may be confined to loss of hair from small areas (alopecia areata). It is due to a trophoneurosis of the cutaneous nerves, which causes atrophy of the hair roots. The conditions which lead to this neurosis are pregnancy, starvation, suckling, spoiled food, and debility. As a rule, in alopecia celsi the hair grows in again, but in alopecia areata the condition often remains permanent and quite resistant to treatment. 
Treatment.-Stimulating liniments applied with friction to the skin, such as one part tincture cantharides, five parts spiritus vini rectificatus or linimentum saponis and spiritus camphoræ equal parts, or liquor ammonii fortior one-half dram and spiritus rectificatus, eight ounces.

\section{Acne}

Acne is a suppurative inflammation of the follicles of the skin arising from the accumulation and decomposition of the sebum secreted by the sebaceous glands, and the invasion of pyogenic organisma.

The disease is generally localized upon the skin of the nose between the anterior nares and the forehead.

Symptoms.-At first, signs of superficial inflammation are evident, then as the deeper follicles become infected the skin appears thickened and upon pressure discharges at various points a bloody, purulent matter. Nodules the size of a pea are also present, which show purulent centers. By confluence of the acne pustules and infection of the hair follicles large abscesses are formed, portions of the skin sometimes becoming even necrotic. In rare cases acne is found on the forehead, trunk, and extremities, in which case the prognosis is very grave.

Treatment.-Incision and free evacuation of the acne pustules and abscesses, and disinfection with tincture of iodin or Lugol's solution, are advised. In persistent cases an autogenic bacterin should be prepared and injected hypodermically every five days. 
INFECTIOUS CONSTITUTIONAL DISEASES

\section{Distemper}

THIS is the most widespread, the most common, 1 the most fatal, and with the exception of rabies the most dreaded malady of dogs. When first discovered in France, from which country it was imported into Great Britain, it was and is still named distemper, yet that name seems unhappily chosen as being too indefinite for correct application to a disease marked by such rarying phases. Distemper is also known as "dog. ill", in some parts of England, and the Scotch term it "the snifters." This latter term, although unscientific, certainly graphically conveys to the mind one important characteristic of the disease, namely, the snifting noisehalf-cough, half-sneeze-made by the $\mathrm{dog}$ in his efforts to get ricl of the exudate which accumulates in the upper air passages; but "snifting" is a term too limited to adequately describe a disease which has well been called the "scourge of the kennel," and which assumes so many forms and complications.

Etiology.-Until quite recently the causative agent of this disease was simply a matter of speculation, many theories being advanced as to the etiological factor. The microörganism now definitely determined as the sole cause of distemper is the Bacillus bronchisepticus**, discovered and isolated in pure culture by Dr. N. S. Ferry. Although responsible for the primary symptoms of the disease, the Bacillus bronchisepticus is by no means responsible

* Many able observers have been unable to verify the findings of Ferry, and the causative factor in canine distemper may be said to be unsettled in the minds of many bacteriologists, not a few of whom believe the agent to be a protozoan. 
for the complications which generally ensue during the course of the malady. There are always closely associated with it the varions strains of Staphylococci and Streptococci, and it is the cffect of their combined toxins that gives us the clinical picture which an animal presents when suffering from distemper. Among the predisposing causes of distemper may be mentioned youth, unhygienic surroundings, in-breeding, in fact, any circumstance which tends to lower the animal's vitality and its powers of resistance to disease or unfavorable conditions.

Symptoms.-The symptoms of distemper vary considerably according to the particular local complications which are developed; they are also dependent upon the sererity of the attack, the powers of resistance of the patient, the rapidity with which the disease progresses and the treatment the patient receives. As a rule, the first obserrable symptoms are pronounced lassitude and dullness, a great disinclination to play or exercise, a decided preference for warmth, the dog creeping into the warmest corner or crouching before the fire, and a general languor that appears to benumb the dog's energies; so that the hitherto lively dog, instead of jumping with delight at his master's call, merely replies with a spiritless wag of his tail and a dismal, woe-begone look. Loss of appetite is an invariable symptom and feverishness ensues, as shown by the hot, dry nose, rigors, and by the clinical thermometer; considerable thirst is present; the bowels are generally deranged, sometimes relaxed, sometimes constipated; the urine is scanty and high-colored; the coat is usually rough and staring; retching and vomiting often occur; there is a thin, watery discharge from the nose and eyes, and a hyperemic condition of the 
conjunctiva; and the eyes appear unusually sensitive to light.

A short, dry, husky cough and sneezing occur, especially when the animal is brought into the open air. The discharge from the eyes and nose gradually becomes more purulent, sticking the nostrils and eyelids together, causing the patient much discomfort and inconvenience and interfering with respiration, and resulting in constant efforts to clear the nostrils which produce that peculiar noise that has earned for the disease the popular name above mentioned. In many cases the eyes are seriously affected.

A small bluish-white opacity may be observerl which gradually widens and deepens until an ulcer is formed. This ulcer, which, by perforating the anterior layers of the cornea, may cause the condition known as staphyloma or may even allow the aqueous humor of the eye to escape. Such cases, although of alarming appearance, usually do well under appropriate treatment, although in some cases of extreme severity some slight opacity of the cornea may remain. As the progress of the disease advances, special symptoms present themselves, depending upon what-organ or organs are chiefly involved. However, a constant and unvarying symptom in all cases of distemper, irrespective of local complications, is rapid emaciation and loss of strength.

In cases where the respiratory tract is chiefly involved the symptoms of bronchopneumonia predominate. When the digestive tract is the subject of serious invasion, digestive disturbances are emphasized and there is vomiting, profuse watery and offensive diarrhea, and in many instances an icterus. When the central nervous system is involved 
symptoms of cerebral congestion, accompanied by convulsions and sometimes by attacks of mania closely resembling those of rabies, make their appearance. Again the patient may become partially or even completely paralyzed, or it may develop the persistent clonic convulsion of some group or groups of muscles, known as chorea.

In distemper the skin, especially that inside the thighs, on the chest, and on the belly, is often the seat of a pustular eruption (the exanthema of distemper). These pustules discharge their contents, dry up, and form scabs. The scabs later on fall oft, leaving small depigmented areas.

The significance of the appearance of this exanthema is still a debated question among practitioners, some contending that it foreshadows a fatal termination, others that its appearance is a favorable symptom. The author's experience is that it is merely a manifestation of the disease and has no significance either one way or the other.

Another prominent symptom in distemper is the characteristic and exceedingly offensive odor emanating from the patient's exhalations and from the skin, the latter also having a peculiar greasy feel to the touch which can hardly be mistaken when once experienced.

Treatment.-The therapeutic indications for distemper vary with the particular complications the case presents. 'In other words, symptoms must be treated as they arise, but the whole rationale of treatment may be summed up by saying that the patient's powers of resistance must be raised to resist the invading organisms, and free elimination must be established to carry off both the microbic toxins and those toxins produced by the normal flora of the intestinal canal, which in discase are 
absorbed into the system because of imperfect elimination and perverted metabolism. Thus we have to deal not only with the toxemia produced by the primary invading organism, but also with the condition known as autointoxication or aututoxemia.

Elimination may be secured by medicines causing free evacuations of the bowels and by those stimulating the free excretion of urine. To obtain the former, a dose of calomel should be given, preferably in repeated small doses to effect; the latter may be attained by appropriate doses of such diuretic medicines as spirits of nitrous ether, potassium nitrate, potassium acetate, or potassium citrate.

In addition to this cleaning-out process, the flora of the alimentary tract must be kept under control by the use of intestinal antiseptics, such as calcium creosote, the sulphocarbolates of zinc, calcium, and sodium advantageously combined with a bismuth salt, acetozone, (fifteen grains to the quart of water and given ad libitum in the drinking water), creosote, or salol in enteric-coated pills or capsules.

To raise the patient's powers of resistance a bacterin composed of Bacillus bronchisepticus combined with Staphylococcus, atreus and albus, must be administered hypodermically every three days in ascending doses. The action of the bacterin treatment is much increased, especially if the patient has not come under observation until the disease is well advanced, if a simultaneous hypodermic injection of nuclein is arministered. The respiratory complications must be treated as they arise, under the directions given previously for handling respiratory diseases (see pp. 12-30). 
A useful combination for general routine of cases is as follows:

R Glycoheroin ..................... iiss

Nuclein solution ................... iiss

Potassium citrate .................dr. ii

Aqua q. s. ...................... $x$

M. Sig.-Give four drams twice daily.

Gastrointestinal complications are treated with the usual agents for the control of romiting, diarrhea, and intestinal fermentation.

The nervous symptoms should be controlled by ice packs applied to the head, if cerebral congestion occurs, followed by the internal administration of bromids, preferably strontium bromid in five tc twenty-grain doses, since this salt is less liable to create gastric disturbance than is potassium bromid Chorea* is best treated by the prolonged administration of Donovan's solutiont, and paralysis is most successfully combated by strychnin and electrical massage. The exanthema requires only the application of an antiseptic wash, such as chinosol (11000 ).

The eyes require treatment from the onset of the disacse. All discharge must be wiped away with cotton-wool swabs soaked in a warm solution of boracic acid and a few drops of a five-per-cent solution of protargol dropped into the conjunctival sack. When ulcerations occur a ten-per-cent solution of protargol should be used, or the ulcers should be touched lightly with a stick of silver nitrate. Any opacity left may generally be removed by the

*Recently considerable publicity has been given to the hypodermic use of a normal brain (rabbit) emulsion in the treatment of chronic cases of chorea. Its value in this connection has not yet been determined.

tDonovan's solution ccnsists of arsenic iodid and mercuric iodid, of each one part, in 100 parts of distilled water. 
daily application of a one-per-cent yellow oxid of mercury ointment.

Nursing and Diet.-Good nursing and an appro.. priate diet form one of the most essential features of the successful treatment of distemper. Without them the most scientific and rational treatment is seriously handicapped, if not entirely useless.

It must br remembered that, even with the bacterin treatment, distemper will run its course either to a favorable or fatal termination, and that therefore no effort must be spared to sustain the patient's vitality during the progress of the disease.

Good nursing consists in seeing that the patient is kept in sanitary surroundings; that he has before him at all times a plentiful supply of clean, cold water; that his eyes and nose are kept clear of discharge and that his teeth and mouth are cleaned at least once daily with some antiseptic wash. This latter point, although generally overlooked, has a most important bearing on the amount of nourishment the patient can be coaxed to take. A dirty, furred-up mouth and unclean teeth are the cause, in a great many instances, of the animal's refusal of all nourishment. It is also the nurse's duty to administer the prescribed medicines and diet, as directed by the attending veterinarian.

The diet for a dog suffering from distemper must be appetizing, easily digested, and easily assimilated. The patient should be fed four or five times a day, small quantities at a time. In the case of a severe attack, with great prostration and weakness, it is necessary to compel the patient to take nourishment by drenching him with beef tea, raw eggs, milk, and so on. Any of these liquid foods may in these cases be advantageously combined with stimulants, such as small quantities of whisky, brandy, or port wine. 
The diet should consist of raw meat minced fine, or, if the patient prefers it, of cooked meat, milk, eggs, and beef tea. Great care must be taken not to upset the patient's digestive system, and no indigestible articles of diet should be allowed.

When convalescence sets in, as shown by the improved appetite, subsidence of symptoms, and raised spirits, a good tonic must be administered (triple arsenates).

Prophylaxis.-Owing to the extreme contagiousness of the disease, great care must be exercised to prevent its spreading. The patient should be isolated, all articles coming in contact with him, such as dishes and water troughs, should be sterilized by boiling immediately after use. All brushes, bedding, sponges, swabs and other articles should be burned, and upon recovery the premises must be thoroughly disinfected. The attendant should sterilize thermometers, pill guns, and medicine spoons before using them again; he should wear a gown while handling the patient, and should disinfect his hands and boots before proceeding to the next case. It is advisable to immunize all dogs exposed to the infection with a prophylactic dose of distemper bacterin.

\section{Rabies}

Rabies is a true infective disease and is transmitted solely by inoculation, generally by the bite of an affected animal. Noguchi, of the Rockefeller Institute, and Zell, of Chicago, simultaneonsly and independently have recently isolated and cultivated the cause of rabies, which appears to be a protozoan, present in the tissues of the central nervous system, saliva, and urine. The virus is fixed and endogenous, that is to say, the animal body is absolutely necessary for its natural development. The period 
of incubation varies from three to six weeks or longer, with an average of twenty-five days.

Symptoms.-The clinical appearance of rabies occurs in two forms, namely, furious rabies and dumb rabies. The two are only different forms of one and the same disease, not two different diseases as was formerly supposed. It must be understood, however, that there are many intermediate forms between these two varieties, which often merge so closely into each other that a distinction is impos. sible. Rabies runs an acute and invariably fatal* course in from two to ten days.

\section{Furious Rabies}

There are three stages recognized in furious rabies,-the premonitory stage, the maniacal stage, and the paralytic stage.

The premonitory stage lasts on an average from twelve to forty-eight hours and is characterized by an alteration in the behavior and disposition of the patient. The animal becomes either morose and sullen, furtive and irritable, or shows just the opposite characteristics-an increased affection and desire for its master's notice. Restlessness and nervousness, and a capricious and perverted appe. tite, are well-marked symptoms of the disease. In some cases hyperesthesia in the region of the site of inoculation causes the patient to bite at the seat of injury. There is a tendency to gnaw and tear up whatever comes across his way, and he sometimes swallows foreign objects, such as stones, earth, grass, rags, pieces of wood, and even excrement. In other cases the patient continually licks his genital organs and shows sexual excitement.

\footnotetext{
*It is believed by some competent authorities that mild cases of rabies occur which are not ordinarily recognized and from which the animal recovers. It is believed that the disease is often spread by these mild unrecognized cases.
} 
In this stage is noticed slight difficulty in swallowing, inclination to romit, dyspnea, and symptoms of choking. Constipation is usually present.

The maniacal stage lasts three or four days and is characterized by attacks of mania and convulsions, with remissions. The patient evinces an overwhelming desire to run away from home, tearing down doors and kennels or breaking his chain in order to effect his escape.

At first the animal shows only a slight tendency to bite, running along aimlessly, sometimes for long distances, but later on it begins snapping at imaginary objects, or at other animals or people that are directly in its way, until finally, losing all control and impelled by some uncontrollable impulse, it savagely and aggressively attacks everything in sight. In some cases the patient may bite and tear its own body, being apparently insensible to pain. A prominent symptom in this stage is the alteration in the voice, which manifests itself as a peculiarly hoarse, howling bark, the first notes of which are prolonged into a high-pitched, longdrawn-out how1.

In the paralytic stage the patient is much emaciated. The eyes are deeply sunken, staring, and glassy, and there is usually external strabismus of one eye. The patient now becomes gradually paralyzed, swallowing becomes impossible, and the saliva runs out of the mouth in ropy masses. In time comes paralysis of the lower jaw, which hangs down, allowing the tongue to protrude. This condition is followed by paralysis of the hind quarters which manifests itself in staggering, stumbling, an:l by increased bodily weakness. The muscles of the tail, rectum, and bladder become paralyzed; and symptoms of great depression are alternated with paroxysms of excitement that gradually become 
rarer and rarer, but which, throughout the course of the disease, are increased in violence by the sight of another dog. Finally the paralytic condition becomes complete and the animal's sufferings are ended by death.

\section{Dumb Rabies}

Dumb rabies is distinguished from the furious type by the early onset of the paralytic symptoms and the absence of the desire to bite unless greatly aggravated. The patient early becomes dull and listless and often appears to have some slight difficulty in swallowing; anorexia is always present. It is in cases of dumb rabies that the veterinarian needs to use much caution when called upon to examine an animal that is supposed to have a "bone in its throat." The paralysis of the lower jaw takes place quite early in the course of the disease and is rapidly followed by the general paralytic condition described above, death usually taking place about the third day.

Treatment.-The disease being quite unamenable to therapeutic measures, any treatment is useless* in either the furious or the dumb forms, but valuable animals may be saved before symptoms have developed if given the Pasteur treatment as soon as possible after being bitten by a rabid animal.

\section{Tetanus}

Tetanus is a specific infective disease, characterized by tonic muscular spasms and caused by the Bacillus tetani. The spores of the Bacillus tetani are ubiquitous and gain entrance to the animal's

*Quite recently several recoveries from rabies, brought about by the hypodermic use of quinin or quinin and urea hydrochlorid, have been reported by doctors of human medicine; however, in inoculated cases these drugs have apparently had no influence on the course of rabies. A serum treatment (Zell) for rabies is being investigated at the present time. 
system through wounds of the skin, however slight. The anaërobic properties of this bacillus make punctured and penetrating wounds the most favorable environment for its derelopment. Suppurating wounds are also favorable to the development of the tetanus bacillus on account of the oxygen being used up by the pyogenic organisms, so that the deeper portions of the wound may be devoid of oxygen, thus allowing anaërobic organisms to develop. The organisms remain at the site of inoculation, the symptoms being produced by the action of their katabolic products or toxins on the nerve centers.

Tetanus is unusual in dogs, this animal being rather less susceptible to the disease than is man and far less susceptible than the horse.

Symptoms.-The general symptoms of tetanus: are a greatly increased irritability of nervous reflex and tonic spasms of the various muscular groups. Trismus, or spasms of the masticatory muscles, occurs, causing the jaws to be firmly held together, hence the popular name of "lockjaw." The recti occulorum, when affected by spasm, cause the eyes to retract within their orbits and the membrana nictitans to protrude over the eyeballs. The whole body becomes rigid; the extremities stiff and stiltlike, the neck outstretched, and the tail elevated. Breathing is interfered with by the spasm of the inspiratory muscles.

Depending upon which group of muscles is mainly affected, the patient's body assumes various positions when the spasms are present. The head and tail may be elevated and the back depressed: the back may be arched and the abdomen tucked up; or the body may be curved laterally. The temperature is at first only moderately elevated, but in cases approaching a fatal termination it may 
reach 108 to 110 degrees Fahrenheit and continut: to rise for some time after death.

The prognosis should be guarded. As a rule it is slightly more favorable in the dog than in the horse.

Treatment.-Good nursing and the avoidance of all excitement and noise are essential. The patient should be placed in a darkened room and kept absolutely quiet, as on account of the extreme irritability of the nervous reflexes the slightest sound or movement may induce or aggravate the spasms.

Nerve sedatives are the drugs indicated, in order to endeavor to control the spasms, the most valuable being gelsemium and lobelia. In exceptionally acute cases chloroform combined with H-M-C in full doses must be used to induce complete anesthesia in order to relax the patient. It must be remembered that to produce the desired effect tetanus patients require doses far in excess of those usually administered, and that these drugs may be pushed with safety. Elimination per anum and through the kidneys must be provided for, and enemas of predigested milk or beef given to sustain life, if the patient is unable to eat.

Tetanus antitoxin is useless as a curative, but as a prophylactic is of undoubted benefit if administered before the appearance of symptoms.

\section{Anthrax}

Anthrax is an infective disease caused by the Bacillus anthracis. Young dogs are extremely susceptible to anthrax, but mature animals appear to develop a comparative immunity. Lating meat containing anthrax germs is the usual cause of the disease in the dog. On this account the animal ordinarily suffers from the intestinal form of the 
disease and from local anthrax of the mouth and pharynx.

It must be remembered that the blood of an infected animal is the medium of infection and also that while the bacilli themselves are comparatively easily destroyed, that the spores are extremely resistant to the action of disinfectants. The gastric juice has no effect upon the spores of anthrax, although it is bactericidal for the bacilli themselves.

Symptoms.-Suddenness of attack, high fever, dyspnea, riolent intestinal disturbances, hemorrhages from all the natural openings of the body, the local manifestations in the mouth and pharynx-salivation, the presence of vesicles on the mucous membrane, swelling of the throat, cyanosis, are all indicative of this disease. The diagnosis is completed by the microscopical examination of the blood and the identification of the Bacillus anthracis.

Treatment.-Needless to say, suspected cases should be handled with extreme caltion, as the disease is communicable to other animals and man. All blood and discharges should be carefully disinfected and the carcasses should be cremated. Attempts to cure the patient are futile.

\section{Tuberculosis}

Tuberculosis is not a common disease of the dog, but cases do occur and such can nearly always be traced to infection from mankind. It has been amply proved that the paticnt becomes infected by inhaling the dust from a room inhabited by a tuberculous person, or by licking up tuberculous sputum or from eating food from the plate of a person affected with tuberculosis.

Symptoms.-Usually the disease assumes the aspect of a chronic pneumonia or a chronic bronchial 
catarrh, quite unyielding to treatment. The temperature is fluctuating, there usually being a rise towards night. There is often weakness and a rapid, progressive emaciation. Auscultation reveals areas of dullness, the presence of cavities, rales, suppressed respiratory murmur and so on.

Diagnosis is assisted by the history of the case and a knowledge of the owner's state of health. It may be confirmed except in advanced cases by the tuberculin test.

Treatment.-Owing to the danger to human beings, treatment should not be attempted. The patient should be sent to the happy hunting grounds immediately.

\section{Granuloma}

This is a disease of true infection, a venereal disease of dogs, affecting chiefly the mucous membrane of the vagina and of the penis and prepuce. It is transmissible by coition and by inoculation.

Cause.-The causative agent is unidentified as yet.

Symptoms in the Female.-Although the lesions are usually confined to the genital organs, other parts of the body may become affected in conjunction or independently. The lesions are generally found on the skin of the neck, throat, back, groin, abdomen and limbs.

Metastatic growths are also often found in the abdominal and mesenteric lymphatic glands, kidneys, spleen, liver and lungs.

In the genital organs of the bitch the lesion generally begins as a circumscribed swelling of the mucous membrane, deep red in color and by a gradual elevation of the tissue and develops into round nodular sessile tumors, these become lobulated, presenting a warty and cauliflower-like appearance. 
These growths are friable and bleed frecly upon the slightest manipulation.

They are most commonly situated in close proximity to the urethral opening. The presence of these-infective venereal tumors is usually unsuspected until a sanious, muco-purulent discharge is noticed at the vulva, when an examination reveals their presence.

In severe cases the whole vagina may become blocked up, rendering coition painful and parturition impossible. After about three months retrograde changes take place in the tumors which break down and disappear but invariably leave the vaginal wall inelastic by reason of cicatricial contraction, so that if numerous parturition is attended with grave difficulties.

Treatment.-The growths should be removed by dissecting them off the vaginal walls, their bases freely curretted and painted with tincture of iodine. Hemorrhage is easily controlled by packing the vagina with aseptic gauze.

Symptoms in the Male.-Hemorrhage after coition, refusal to serve and sometimes difficulty in urination (if the growth involves the urethral opening) should lead to an examination for venereal warts. Exposing the penis by drawing back the prepuce of course reveals the condition.

In the male the infective tumors, in the early stages of the affection, appear as small yellowishwhite raised patches about the size of a mustard seed. Later on the growths take on the characteristic cauliflower-like appearance and bleed readily when touched. They may be situated at the extreme end of the penis, upon the sides or at its base.

Treatment.-Secure the patient in the dorsal po- 
sition and then fill prepuce with a five per cent solution of cocaine. After waiting ten minutes to allow the parts to become properly anesthetized, expose the penis, pick up the growths with rat-tooth forceps and snip them off with sharp scissors, cutting well into the surrounding healthy tissue. The resulting wound should be treated antiseptically and, if extensive, sutured with fine silk (No. 00). 


\section{INTERNAL PARASITES AND PARASITISMS}

Contrary to the popular belief, intestinal parasites, unless the infestation is particularly heavy, do not cause any very serious constitutional disturbances. However, large numbers of parasites, especially in puppies, may give rise to alarming symptoms and in some instances cren cause death. Intestinal parasites are detrimental to their host by reason of the irritation they produce, their interference with the digestive process, the deleterious effect of their waste products, the abstraction of blood, or by mechanical obstruction. We may, therefore, look for a definite train of symptoms when the infestation is heavy.

The general symptoms of parasitism are a general unthrifty condition of the patient-a rough, dry coat, appetite variable but usually voracious, anemia, gradual cmaciation and alternate constipation and diarrhea. Convulsions are common and in certain special cases there is bleeding from the nose. Pruritus ani is well marked in cases where the parasites inhabit the rectum. The diagnosis, however, is positive only when the parasites or their ova are detected in the feces. Notwithstanding the comparative harmlessness of parasitism to the patient itself, every means should be employed to rid him of these pests and to destroy their ova and thus prevent their reproduction, on account of the danger from some of them to both man and other animals. Of these Trenia echinococcus and Tænia cœnurus are examples producing respectively hydatid disease 
in man and "gid" in sheep. Only brief reference to the morphology and life history is given here, the student being referred to the standard works on parasitology for more detailed information.

\section{Spiroptera Sanguinolenta}

This parasite is a small, round worm that inhabits the walls of the esophagus and stomach. The intermediate host is the Blatta orientalis. This nematode worm is readily recognized by its bloodred color, and in addition to its normal habitat as stated above, it may be found in the aorta, lungs, and lymphatic glands. Its presence in the esophagus and stomach forms tumors consisting of indurated tissues in which there are chambers containing the rolled-up worms.

Symptoms.-Repeated voniting and rapid emacidtion give evidence of infestation by this parasite. Sometimes, too, there is rabiform convulsions.

\section{Ascaris Marginata}

The Ascaris marginata is a nematode worm, the male from five to ten centimeters long, the female from nine to twelve centimeters long. In color it is white or reddish white. These worms are more especially common in puppies, who may be infested with them at three or four weeks old, but it is at the age of three or four months that they are most abundant. Their habitat is the small intestine and frequently the stomach.

Symptoms.-Unthrifty coat, cmaciation, enlarged abdomen (pot-belly), variable appetite, sometimes convulsions, colics, and constipation or diarrhea result from infestation by this parasite. By collecting in rolled-up masses the parasites sometimes cause complete intestinal obstruction, which is followed by death. In one case in the author's practice the 
stomach and intestines of a month-old puppy were found on postmortem examination to be tightly packed with worms, which, when removed, completely filled a six-ounce bottle.

Treatment.-All solid food must be withheld for twelve hours, when capsules containing calomel and santonin, one-half grain of each, should be administered every three hours until purgation takes place. The following prescription has given complete satisfaction to the author in numerous cases:

I; Fluidextracti spigeliæ,

Fluidextracti sennæ, aa............. i

Sig.-Give 15 to 30 minims every morning until three doses have been given.

There are many other vermifuges applicable, but whatever one is used, a laxative should either be combined with it or administered after the last dose, and in every case of worm infestation the treatment should be repeated every two weeks until the feces show no evidence of parasites or of their ova. All feces of infected dogs should be burned to prevent other animals from becoming infested.

\section{Oxyuris Vermicularis}

This is a small, white, thread-like worm, the male from two to three millimeters long, the female from nine to ten millimeters long. These parasites inhabit the rectum and cause considerable irritation and anal pruritus; the patient continually drags the anus along the floor to relieve the itching.

Symptoms.-Anal pruritus and the presence of the parasites in the feces prove the presence of the parasite.

Treatment.-A laxative should be administered, followed by enemas of a saturated solution of sodi$11 \mathrm{~m}$ chlorid or a strong infusion of quassia chips. 


\section{Ankylostomum Trigonocephalum}

The hookworm is white in color, the male from nine to tivelve millimeters long, the female from nine to twenty-one millimeters long. These parasites inhabit the small intestines and produce grave symptoms. They are true bloodsuckers, producing pernicious anemia.

Symptoms.-The symptoms are those of grave anemia-a gradual but progressive emaciation, and debility. The appetite is unaffected at first, but later on becomes capricious and finally disappears. The coat is staring, the skin scaly and dry. The nose is tumefied and becomes excoriated with the mucosanguinolent discharge. Later a copious epistaxis supervenes, two or three ounces of blood being lost at each attack. These attacks of epistaxis recur at intervals of a few days to several weeks, during which the mucosanguinolent discharge continues.

In some cases convulsions occur. Intermittent edematous swellings make their appearance on the limbs, which finally become permanently edematous. In the last stage there are ulcerations, gangrenous patches, and persistent diarrhea, which may give place to dysentery. The course of this condition is extremely chronic, the animals lingering for months or even a year.

Treatment.-iVhen pernicions anemia appears in a pack of hounds extreme care should be taken to thoroughly clean and disinfect the kennels. The sick should be isolated and prevented from soiling the drinking troughs, they should be fed and watered in individual utensils which should be boiled immediately after use, and their feces should be burned.

The anthelmintic which has been proved to be 
of the greatest service is thymol given in large doses and followed up by a saline laxative. Oil of male fern is also very effectual. This latter should be given only after the patient las been fasted for twenty-four hours, having previously received a purgative. Six to eight hours after the exhibition of the male fern a saline purgative should be given to clean the intestinal canal. The strength of the patient must be carefully maintained by nutritious and easily digested foods-milk, and raw meatwith tonics of iron and a bitter tonic such as quassia or gentian.

\section{Tricocephalus Depressiusculus}

A white worm forty-five to seventy-five millimeters long. This parasite inhabits the cecum of the $\operatorname{dog}$ and is often found in conjunction with the Ankylostomun trigonocephalum. It produces anemia and often typhlitis-appendicitis.

Treatment.-The same as for Ankylostomum trigonacephalum.

\section{Tænia-Tapeworms}

The tapeworms most commonly. met with in the dog are merely mentioned here and the reader is again referred to works on parnsitoingy for a more extended description. The fulluwing table gives the name, the intermediats: host, and the larval form:

\begin{tabular}{|c|c|c|c|}
\hline Name & LENGTII & $\begin{array}{l}\text { INTERMEDIATE } \\
\text { HOST }\end{array}$ & Larval Form \\
\hline Tenia Serrata.... & $50 \mathrm{~cm}$. to $2 . \mathrm{m} .$. & Hare and rabbit.. & Cysticercus pisi- \\
\hline $\begin{array}{l}\text { Tania Serialis.... } \\
\text { Tania Marginata. }\end{array}$ & $\begin{array}{l}45 \mathrm{~cm} \text {, to } 75 \mathrm{~cm} \text {. } \\
1.5 \mathrm{~m} \text {. to } 2 \mathrm{~m} . . .\end{array}$ & $\begin{array}{l}\text { Rodents } \ldots . . . \\
\text { Ruminant } \ldots\end{array}$ & $\begin{array}{l}\text { Conurus serialis } \\
\text { Cysticercus tenui- } \\
\text { collis }\end{array}$ \\
\hline $\begin{array}{l}\text { Trnia Coenurus.. } \\
\text { T. Echinococcus.. }\end{array}$ & $\begin{array}{l}50 \mathrm{~cm} \text {. to } 75 \mathrm{~cm} . \\
4 \mathrm{~mm} \text {. to } 5 \mathrm{~mm} .\end{array}$ & $\begin{array}{l}\text { Sheep . . . . . . . } \\
\text { Man, sivine, rumi- } \\
\text { nants........... }\end{array}$ & $\begin{array}{l}\text { Conurus cerebralis } \\
\text { Echinococcus poly- }\end{array}$ \\
\hline Trenia Canis...... & Io $\mathrm{cm}$. to $40 \mathrm{~cm}$. & Louse and flea... & $\begin{array}{l}\text { morphous } \\
\text { Cryptocystus trich- }\end{array}$ \\
\hline 'Trenia Krabbei.... & I $171 \ldots \ldots \ldots \ldots$ & Reindeer & $\begin{array}{l}\text { Cryptocystus serra- } \\
\text { ticeps }\end{array}$ \\
\hline
\end{tabular}


Of these tapeworms the most important from an economic standpoint are the Trenia echinococcus and the Trenia cœnurus, the first producing hydatid disease in man, the second "gid" in sheep.

The symptoms of tapeworm infestation are those of general parasitism, and the diagnosis is positive when the segments are found in the feces.

Treatment.-After thoroughly preparing the animal by fasting and laxatives, administer on an empty stomach fifteen minims to one dram of oil of male fern, followed in eight to ten hours by a saline cathartic in full doses. The expelled parasites should be carefully examined to ascertain if the head has been expelled, and if not the treatment should be repeated in ten days. The same precautions as to burning worm segments and the bowel discharges must be taken as mentioned before in this section. 


\section{POISONS AND POISONING}

P OISONING in the dog may be accidental or intentional. The former may come about by the animal picking up poisoned meat or other food laid down for vermin, or the patient may show symptoms after an overdose of some drug given empirically. Some animals show a great susceptibility to the action of some drugs, notably strychnin, symptoms of poisoning appearing after even moderate medicinal doses. Poisons cause death

1. By their corrosive or tissue-destroying properties;

2. By their physiological action;

3. By a combination of (1) and (2).

Poisoning in canine patients, whether intentional or accidental, is only too often fatal, the quantity of the drug ingested in nearly every case being large and the patient's condition often not discovered until irreparable damage has been done. The usual portals through which poisons are absorbed are the respiratory and digestive system, with air or food. Occasionally they are absorbed through wounds in the skin.

\section{Pathology of Poisoning}

Local Effects.-These are the results of the action of the poison on the living cells with which it comes in contact and consist of more or less violent irritation, varying in degree from congestion or inflammation to mortification, and these local effects react on the whole system, giving rise to fever, collapse, and other serious conditions. 
Absorption.-The rapidity with which poisons are absorbed depends to a great extent on the poison itself, the portal by which it gains entrance to the animal's economy, the condition of the digestive tract-whether full or empty, and also the nature of the stomach contents. For instance, the unbroken skin will casily and rapidly absorb gases, but solids or liquids hardly at all; on the other hand, irritated or woundea skin becomes very absorbent. Again, an empty stomach will absorb far more readily and rapidly than a full one, and yet again, the digestive tract just after a meal of fatty substances, even milk, is far more impenetrable than when a meal with no fat has been partaken of. The exception to this is phosphorous, the absorption of which is accelerated by oils and fats.

Elimination.-Poisons are eliminated in the excretions. Nearly all mineral poisons are eliminated through the kidneys, some by the bowels. Gases, vapors, and volatile poisons are eliminated by the lungs. Some poisons undergo changes during elimination, others are eliminated unaltered.

Treatment. - The main objects to be attained are to delay or prevent absorption, to neutralize the poison or render it insoluble, to eliminate from the system both what is absorbed and what is unabsorbed, and to counteract the effects produced by the poison absorbed and the resulting lesions.

The first can be accomplished by emetics and purgatives, the former by reason of their rapidity of action being the most valuable. Purgatives are chiefly valuable when combined with agents that retard absorption or with those that unite with poisons and form insoluble or inert compounds. Antidotes act in three ways: they neutralize the poison, render it insoluble, or arrest its injurious effects. 


\section{Arsenic}

Arsenic, or rather arsenious acid (arsenic trioxid), is one of the commonest of vermin poisons, and dogs are frequently poisoned on that account. Dogs may also become poisoned by the arsenic in an overdose of Fowler's solution given for medicinal purposes.

The toxic dose of arsenic is two grains. Arsenic is a corrosive irritant, producing gastroenteritis, with its corresponding effects.

Arsenical poisoning may be either acute or subacute.

Symptoms of Acute Arsenical Poisoning.-About an hour after ingestion there suddenly appears profuse salivation, swallowing movements, and great thirst. The patient is restless and anxious, with convulsive action of the lips, acute colicky pains, and vomiting. There is great pain over the epigastric region, and intolerance to pressure. At first there is constipation, which later on gives way to a fetid dysentery. The pulse at first is full, but soon becomes small, irregular, and imperceptible; respiration is difficult, urine scanty and often bloody, and the temperature is subnormal and the extremities cold. Later on rigors set in, with weakness and paresis, the gait is staggering, the pupils dilated. The pain is excruciating, and the animal dies in a state of collapse in from four to five hours.

Symptoms of Subacute Arsenical Poisoning.-There are much the same as in the acute form, but not so violent. There is dryness of the mouth, great thirst, colicky pains, vomiting, fetid diarrhea, and tympanites. The mucous membranes are pale, the pulse irregular but frequent, the urine scanty. These symptoms may persist for about twenty-four 
hours, when the patient seems to improve. This improvement, however, is short-lived, the patient soon relapsing and becoming worse. There is great depression and weakness and he is unable to stand. Dyspnea is present, and the pulse is weak and almost imperceptible. Convulsions may occur, or the patient may lapse into coma. There may be edema of the eyelids and dependent parts; paralysis of the hind quarters then sets in, and death follows in a day or two.

Treatment.-Administer an emetic, $1-20$ to 1-10 grain of apomorphin, and follow with an antidote.

Antidote: Sesquioxid of iron prepared by precipitating tincture of iron perchlorid with an alkali. Large amounts of this antidote should be given every twenty minutes or so, followed up by at purgative of magnesium sulphate. Three ounces tincture of iron perchlorid, and one ounce of sodium carbonate, dissolved in water and mixed together, will produce sufficient sesquioxid to neutralize ten grains of arsenious acid. Insoluble arsenate of iron is formed and is swept out by the magnesium sulphate.

In acute cases hypodermic injections of morphin every half hour will retard absorption and ease pain. Later on and in subacute cases, opium combined with a demulcent has the same effect and tends to soothe the inflamed mucous surfaces. To hasten elimination, spiritus etheris nitrosis should be given in full doses, well diluted with water. Collapse must be combated wth stimulants.

\section{Phosphorus}

Phosphorus poisoning in dogs is usually accidental, and due to their picking up pieces of bread or meat smeared with vermin paste of which phosphorus is the chief constituent.

Symptoms.-The first symptoms of phosphorus 
poisoning generally appear about an hour after the ingestion of the poison, manifestations of acute gastritis, increased flow of saliva, acute pain, bloody diarrhea, extreme prostration, convulsions, and coma occurring in rapid succession. A garlicky odor of breath and icterus complete the clinical picture. If the patient is taken into a darkened room, his breath, feces, and urine appear luminous. If the patient survives the acute symptoms, fatty degeneration, chiefly of the liver, takes place, from which the animal may die later on.

Treatment.-An emetic of two grains of copper sulphate in water should be given and repeated until the stomach is completely emptied. Copper sulphate is both an emetic and an antidote for phosphorus, forming an insoluble phosphid of copper. After all the poison has been ejected by vomiting that it is possible to eject in this way, demulcents and opiates should be given, but on no account should oil or milk be used, as they hasten the absorption of the phosphorus. To counteract the extreme prostration and collapse associated with phosphorus poisoning, subcutaneous injections of strychnin sulphate (gr. 1-200 to gr. 1-60) or one-half to one ounce of spirits of camphor should be given.

\section{Strychnin}

Strychnin poisoning is probably the most common form of poisoning with which the veterinarian has to deal. The dog poisoner generally chooses this powerful alkaloid to attain his nefarious object. In addition, canine patients are extremely susceptible to strychnin and poisoning frequently results from overdosage of this drug. These latter cases, however, are usually quickly amenable to treatment. In malicious poisoning, on the other 
hand, the mortality is high on account of the mas:ive doses employed.

The length of time which elapses before symptoms of poisoning appear after the ingestion of toxic quantities of strychnin varies considerably, depending on the nature and amount of food in the stomach. Absorption is retarded by a full stomach and by food stuffs of a fatty nature. The author has records of a case in which this delayed absorption is well illustrated. The patient on her return home was given a bowl of milk, after which she was under continual observation until the symptoms of strychnin poisoning developed, a period of four hours. The dose in this instance must have been a large one, as the bitch was saved only by heroic doses of H-MI-C, being kept narcotized for twenty-four hours.

Symptoms.-Uneasiness, an anxious expression, and panting, followed by acute tetanic spasms at varying intervals, are indications of strychnin poisoning. The spasms affect the whole body, the limbs become stiffened, and the animal, unajle to stand, falls to the ground. The jaws are in a state of trismus, the lips being drawn back, especially at the commissures, producing the characteristic risus sardonicus. The spine is curved (lordosis), inspiration is labored, and the nucous membranes are cyanotic. The eyes are prominent, the pupils dilated, the heart action tumultuous, and the pulse small and quick, but the artery full and hard. After the spasm the muscles relax and the foregoing symptoms abate until another paroxysm sets in, and these become more and more frequent, with shorter intervals, until ultimately the patient dies from asphyxia during one of the convulsions.

Treatment.-Immediately an emetic of $1-10$ to 1-20 grain of apomorphin hydrochlorid should be 
administered hypodermically to produce prompt vomition of the unabsorbed portion of the poison. No matter what treatment is resorted to for the purpose of allaying the spasms, this emetic must be given first of all; wnless the quantity of strychnin ingested be very small, all other treatment is futile, and a fatal termination certain if this is neglected.

If the patient can swallow, the emetic may be followed by a liberal dose of oil. To control the spasms, the animal must be chloroformed, or an intravenous injection of chloral hydrate may be given. Large doses of morphin sulphate may also be used subcutaneously; but the treatment that has given the best results, and has in addition the advantage of easy administration, is the hypodermic injection, after the production of emesis, of from three to five tablets of H-M-C (No. 1). The main object is to keep the patient free from spasms until the poison is eliminated. These injections of the H-M-C tablets should be repeated in sufficient dose to attain the result, the procedure being entirely without danger. The patient when completely narcotized should be placed in a dark and quiet place and all noise and excitement avoided,-or a return of the paroxysms will result.

\section{Hydrocyanic Acid and Potassium Cyanid}

These poisons are sometimes used by the dog poisoner, the latter being the more used of the two.

Symptoms.-The rapidity with which this poison usually acts does not allow of the development of definite symptoms, but when relatively small toxic doses have been given (luckily the commercial potassum cyanid is often impure) the following symptoms appear in a few minutes after the ingestion of the - poison: Great distress, difficulty in breathing, head and neck extended, and convulsive movements of 
the fore limbs. The patient next falls on his side and goes into convulsions, the spine being alternately arched (kyphosis) and curved (lordosis). There is champing of the jaws, an increased flow of saliva, spasms of the diaphragm, asphyxia, and death.

Treatment.-Death usually takes place so quickly as to preclude treatment, but if there is time, 1-10 to 1-20 grain of apomorphin hydrochlorid should be given for its emetic effect, and the poison neutralized with a solution in water of ten grains of iron sulphate. Complete anesthesia should then be.induced by the administration of ether.

\section{Carbolic Acid (Phenol)}

Dogs are extremely susceptible to the effects of carbolic acid and are often poisoned by being washe: 1 in a strong solution for the eradication of vermin, or by licking wounds to which carbolic dressings have been applied.

Symptoms.- TVhen taken by the muth the caustic action of carbolic acid is observed by the stomatitis, pharyngitis, and gastroenteritis which it produces. The mucous membranes that have been touched by it appear white in patches. Then phenol is absorbed into the general circulation the following symptoms appear:

The patient is anxious and distressed; there are fits of rigors and convulsions followed by paralysis of the hind quarters; the pulse is small, irregular, and barely perceptible; the respiration is quick and difficult; the temperature subnormal, and the urine high colored.

Treatment.-Alcohol neutralizes carbolic acid and is, in addition, a stimulant. It should be given in full doses, one to two ounces, or if it be obtainable give gin, one to four ounces. A full dose of sodium sulphate should then be given for a purgative. This 
also in a measure neutralizes the poison. The inflamed mucous membranes should be soothed by the administration of oil or demulcents. The failing respiration should be combatted by repeated full doses of atropin sulphate (1-100 to $1-25)$ administered hypodermically.

\section{Iodoform}

Poisoning from this agent is usually due to absorption from wound dressings or from the patient licking wounds that have been dressed with iodoform.

Symptoms.-Gastric disturbances, vomiting, constipation, and convulsions alternating with coma, are produced by the ingestion or absorption of toxic quantities of iodoform. In some cases there is great excitement and difficult breathing. The pulse is irregular.

Treatment.-Eliminate by emetics and give starch to form insoluble starch iodid.

The foregoing list of poisons comprises those usually met with in practice; for a more extended list, the reader is referred to special works on toxicology. 


\title{
PAR T I I \\ SURGICAL OPERATIONS
}

\author{
S E C T I O N I
}

\section{PRELIMINARIES}

THE successful carrying out of an operative pro1 cedure depends partly on the skill of the operator and still more on the rigid adherence to the rules of asepsis and antisepsis. To attain these essentials, some means of restraining the patient's movements must be provided for. There are numerous and varied types of operating tables to be found on the market, but for all practical purposes a perfectly efficient table may be devised at home. An ordinary kitchen table, enameled white, makes a thoroughly practicable and cheap substitute for the high-priced tables sold by the surgical supply houses.

The top of the table should be removed and fixed on again with hinges at one end, to allow the top to be tilted at any angle. At the other end of the framework of the table a square frame is hinged, the top of which butts against slats screwed on the inferior surface of the table top. In this way various angles of inclination may be obtained.

On the table top are screwed four cleats, one at each corner, to which to affix the hopples. The hopples themselves may be made of tape, smallsized cotton rope, or webbing. A loop is made in one end of the hopples and a slipknot formed by passing the other end through the loop. In securing the patient the slipknots are placed on each leg, above the carpus or the tarsus, as the case may be, the patient stretched out, and the ends of the hop- 
ples passed round the cleats in figure-of-eight style. The positions in which the patient is placed are the abdominal and the ventral. In the former the patient, is stretched on the table on his belly; in the iatter, on his back. Occasionally the position of lateral recumbency is required, when the patient is placed on his side and the feet secured to the cleats opposite to each other.

\section{Anesthesia}

To obtain the best results, as well as for humanitarian reasons, all operations (with the exception, perhaps, of evacuating an abscess, which as a rule requires only one bold stroke of the knife) should be performed under anesthesia.

Anesthesia may be either local or general. As a general rule local anesthesia is applicable only in minor operations of short duration. It is applied by a spray to the part to be operated on, or the part is injected subcutaneously, by means of an ordinary hypodermic syringe, with the anesthetic selected. Ethyl chlorid is sprayed on the site of operation until the tissues are nearly frozen. It is of use onfy in such operations as opening an abscess or giving an intravenous injection, because the anesthesia is only fleeting and cannot be pushed on account of the danger of the tissues sloughing from too prolonged freezing. The anesthetics administered hypodermically are solutions of cocain, cucain, urea and quinin hydrochlorid, alypin, acoin, novocain, and stovain. Of these cocain is probably the most used and is quite safe, provided that the amount injected does not exceed three-fourths of a grain. Although solutions of varied strength may be used with the above limitation as to dose, a four-per-cent solution is found to be the most suitable for general work. It must be remembered that 
cocain has toxic properties and that no more than a tenth of a grain per pound weight of the patient should be injected at one time. The addition of adrenalin to the solution not only delays absorption into the circulation and so diminishes the risk of poisoning, but by constricting the capillaries it renders the operative field comparatively bloodlessa great advantage in some operative procedures.

Technic.-The site of operation is shaved and cleaned up as described hereafter, and a sterilized hypodermic syringe is filled with the anesthetic solution. A sterilized needle is introduced under the skin in the usual manner, the syringe attached,

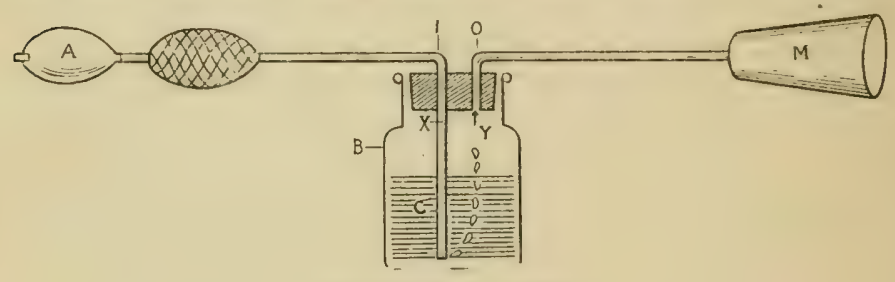

Chloroform Inhaler.

a few drops injected, and the needle withdrawn. Within the wheal produced by the first injection the needle is again introduced and another injection made, and so on until the whole operative area is injected. Gentle massage to the part is then applied for a minute or so in order to distribute the solution. A pause of about fifteen minutes must be made before commencing the operation.

\section{General Anesthesia}

Being so easily and safely induced, general anesthesia in canine practice is by far the most practicable and satisfactory. The general anesthetics most in vogue are chloroform and ether, but various mixtures of these, with or without the addition of alcohol, are often substituted, but with no advan- 
tage to the patient. The safest and must desirable method of inducing general anesthesia is as follows:

About one hour previous to the operation the patient should receive a hypodermic injection of $\mathrm{H}-\mathrm{M}-\mathrm{C}$ (hyoscin, 1-100; morphin, 1-4; catin, 1-64), this usually produces vomiting in ten minutes. The dose must be ganged by the animal's age and general condition, and varies from one No. 1 tablet for a puppy or small dog to five No. 1 tablets for a collie or bull terrier. The anesthetic is then completed by a few whiffs of chloroform administered by means of a Funker's inhaler or some similar apparatus. The advantages gained by using this method are (1) the emptying of the stomach; (2) the small amount of chloroform required to produce profound but safe anesthesia; (3) the long postoperative hypnosis that the patient enjoys, with corresponding benefit to the healing process.

Chloroform alone may be quite safely administered if due considerations are given to the following details :

1. The patient must be prepared by fasting and the exhibition of a laxative twelve hours previous to operation.

2. The anesthetic must be administered slowly and be largely diluted with air.

3. The anesthetic must be administered only in sufficient quantity to inhibit the reflexes.

4. A sharp lookout must be kept for the danger signal, namely, shallow respirations. It. must be remembered that it is the respiratory center that first becomes paralyzed, and that respiratory failure precedes cardiac failure.

5. At the first sign of respiratory trouble the anesthetic must be immediately discontinued, stimulants given, and if need be artificial respiration per- 
severed with antil normal breathing is again established.

6. In case of sudden collapse, every effort must be made to reëstablish respiration. Remove the chloroform mask at once, pull the tongue forward with the tongue forceps, and drop on the back of the tongue the requisite dose of Schcele's acid.* The dose of the acid is one-eighth of a minim for each pound in weight of the animal. The sudden gasping which immediately takes place on the administering of hydrocyanic acid in a large majority of cases starts the respirations again. Then give the patient a smell of strong ammonia and a hypodermic of 1-100 to $1-50$ grain of strychnin, according to its size. If these measures fail, give adrenalin solution (1-1000) hypodermically. In addition to this medicinal treatment, artificial respiration must be resorted to.

Technic-The following apparatus, instruments, and drugs are required to be ready to hand: Chloroform apparatus as illustrated, chloroform, strong ammonia, Scheele's acid (one eighth minim to a pound weight of patient), medicine dropper, hypodermic syringe filled with strychnin solution (1-50 grain), adrenalin solution (1-1000), and tongue forceps.

Administration.-The patient should be secured in the abdominal position on the table and the mask applied over the nose and mouth. The anesthetist then gently squeezes the bulb of the apparatus, taking care to give the patient plenty of time, after the first struggles have subsided, and the patient passes into the second or narcotic stage, recognized by a series of short, sharp yelps or whining. Then the chloroform may be pushed faster until the anes-

*A five-per-cent aqueous solution of absolute. hydrocyanic acid; named for Carl Wilhelm Scheele (1742-1786), a Swedish chemist, discoverer of chlorin and oxygen. 
thetic stage is reached, as shown by the abolition of reflex action. The administration of the anesthetic must then be continued with great caution, the bulb being squeezed only often enough to maintain anestíesia.

If due regard is given to this method of anesthetizing, the patient is kept well within the safety zone and accidents will be of rare occurrence. The author has anesthetized hundreds of cases by this method, which is followed by Professor Hobday, without a single fatality.

\section{Preparatory Treatment of the Operative Area}

This most important preliminary must in all cases be rigidly carried out, as only by surgical cleanliness can healing by primary union be brought about.

Technic.-All hair should be removed by clippers or scissors, the part thoroughly scrubbed with antiseptic soap and boiled water, and then shaved. The site of operation should then be rinsed with boiled water, or rubbed with swabs dipped in ether or gasolin, to remore the greasy secretion of the skin. This must be persisted in until the swabs come away perfectly clean. Tincture of iodin is then applied and allowed to dry, the process being repeated three times. The body of the patient should be covered with gauze wrung out in boiled water, to prevent hairs or dust getting into the wound during the operation.

\section{Preparation of Instruments}

One of the most important factors in the successful healing of surgical wounds is the prevention of pathogenic organisms being introduced either by instruments, sutures, dressings, or the operator's hands. In other words, everything that comes in contact with a wound must first of all be rendered aseptic. Instruments can readily be sterilized by 
boiling for fifteen minutes. A piece of ordinary washing soda should be added to prevent discoloration and rusting. The instruments can then be laid in a sterile dish or instrument tray containing some sterile solution.

The author particularly wishes to emphasize the point that boiling is the only safe method of sterilization, and that putting instruments into an antiseptic solution of a strength that will not injure them is quite ineffective for the purpose of sterilization.

\section{Preparation of the Operator's Hands}

This is one of the most important details that must be attended to, and at the same time the most difficult of accomplishment. The varied duties arising in veterinarian practice make the possession of the ideal soft, white, and sensitive surgeon's hands almost an impossibility. Try as we may, our hands get rough, chipped and cracked, and therefore form a suitable habitat for microörganisms. The safest and best way when operating is to wear rubber gloves, which can easily be sterilized, thereby saving an immense amount of time and trouble. For those, however, who dislike gloves the following routine may be of service: Wear leather gloves while securing the patient, then before operating clean the nails, scrub well with hot water and soap for five minutes, then in alcohol, then in 1-1000 solution of bichlorid of mercury for five minutes, then rinse off in two changes of boiled water. 'It must be always borne in mind that after the hands are once cleaned nothing that is not surgically cleanthat is, aseptic-must be touched. Should this occur, the hands must again be disinfected before touching the wound. Owing to the extreme difficulty of rendering the hands surgically clean, it is best to handle the wound or tissues as little as 
possible with the fingers. With a little practice, nearly all manipulations required can be done with instruments. Take, for instance, the suturing of a wound: the edges are grasped with tissue forceps, the needle held in a needle forceps or holder, and the suture placed without fear of contamination from the operator's fingers. 


\section{SURGERY OF THE HEAD AND NECK \\ Hematoma of the Ear Flap}

TI EMATOMA consists of an effusion of bloo: 11 or serum between the skin and cartilage. It is due to trauma, and first appears as a painful fluctuating swelling on either the under or upper surface of the ear flap, or in some cases on both surfaces. If not dealt with the contents of the sac become organized and by contraction produce a most unsightly distortion of the ear.

Materials for operation.-The following instruments, sutures, and dressings are sterilized and placed in sterile trays ready for use: One scalpel, two pairs of artery forceps, one needle forceps, ten straight needles, ten silkworm-gut sutures, one curette, one dressing forceps, cotton-wool swabs, aseptic gauze, dressing (iodized collodion), aseptic wool (ether), one ear cap or many-tailed bandage, a piece of rubber sheeting ten by twelve inches in size with a slit three inches long in the middle.

Place the patient in the abdominal position and induce general anesthesia.

Technic.-Both sides of the ear flap are shaved and prepared as before described. The external auditory meatus is plugged with cotton wool and the ear pulled through the slit in the rubber sheet, which is laid over the patient's head and neck in order to prevent the operative area touching it.

A longitudinal incision is made in the center of the swelling, sufficiently large to permit of the complete evacuation of the contents, which may 
consist of blood or serum, clots, and tissue debris. The cavity between the skin and cartilage is then gently but thoroughly curretted and any hemorrhage controlled by pressure. The next step is to suture the wound and completely olliterate the carity between the skin and cartilage.

Method of Suturing.-The needle is introduced from the opposite side of the ear flap through the skin and cartilage so that it comes through about one eighth of an inch from the edge of the wound. The suture is taken across the wound and up through the flap again, and tied. The sutures must be placed abont one eighth of an inch apart and be of sufficient number to completely close the wound.

Additional sutures placed in the same manner are then put in where required to obliterate the cavity and insure cluse approxination of the skin and cartilage. The ear is then swabbed with ether, the wound sealed with iodized collodion, and the whole ear enveloped in a sterile gauze and wool dressing, laid on the top of the head, and the ear cap or manytailed bandage applied.

The position of the ear should be changed every twenty-four hours; that is, bandaged over the top of the head or against the cheek. The dressings should be removed on the funrth day and the wound examined. As a rule, with good technic healing is by first intention, but if not the ear is cleaned every day with hydrogen peroxid and dressed with antiseptic dusting powders and dressings, gauze and wool, until healed. The sutures are removed on the seventh day.

\section{Plastic Operation for Split Ears}

Split or torn ear flaps are commonly the result of fighting, and unless remedied give the patient . 
most unsightly appearance and detract considerably from its value.

Experience teaches that simple suturing of the torn parts is most unsatisfactory, since the parts do not as a rule heal properly, the stitches tearing out and the edges of the wound again separating. By using the following technic, however, uniformly good results are obtained to the satisfaction of the owner and surgeon:

Place the patient in the abdominal position and induce general anesthesia, having the following instruments, dressings, and so on ready for use: A small scalpel, dressing forceps, artery forceps, needles and fine silk sutures, iodized collodion, ether, swabs, and gauze.

Technic.-The instruments, needles, sutures, swabs, and gauze are sterilized by boiling and the ear shaved and carefully disinfected in the usual manner. The ear is then pulled through a slit in the gauze so as to prevent it coming in contact with the head or neck during the operation.

After thoroughly disinfecting his hands, the operator then takes the scalpel and dissects the skin away from the cartilage for about one eighth of an inch on both sides of the ear and on either side of the wound. The strips of cartilage thus freed from the skin are then excised, hemorrhage controlled, and the edges of the wound on both sides united with closely placed interrupted sutures. The wound is then swabbed with ether, which is allowed to evaporate, and sealed with the iodized collodion. Healing occurs by furst intention and the sutures are removed in six days.

\section{Operation for Harelip}

Place the animal in the abdominal position and induce general anesthesia with II-M-C and chloroform. 
fior materials to be used have ready a scalpel, scissors, tissue forceps, artery forceps, harelip pins, silk sutures, needles and wire cutters (all to be sterilized by boiling); also the necessary dressings, sterile swabs and iodized collodion.

Technic.-The edges of the cleft are "rawed" up with the scalpel and the mucous membrane uniting the under surface of the lip to the gums dissected loose, so that there is no tension on the lip when the edges of the cleft are approximated. This is the essential part of the operation.

The edges of the cleft are then united by passing the harelip pins through the wound and passing the silk sutures around them in a figure of eight. The pins should be placed about one quarter of an inch apart. The bottom of the cleft at the edge of the lip is united with a single suture and the whole sealed with collodion after cutting off the superfluous portions of the pins with the wire cutters.

\section{Operation for Cleft Palate}

Place the animal in the dorsal position and induce general anesthesia with H-MI-C and chloroform. The following instruments must be at hand: palate knife, scissors, mouth gag, palate needles, silk sutures, needle holder, swabs and swab holder, all to be sterilized by boiling.

Technic.-An incision is made parallel with and on each side of the cleft through the mucous membrane of the palate, which is then loosened from the bone with the points of the scissors (Chury's operation). The object of this is to enable the operator to approximate the edges of the cleft without tension on the sutures.

The edges of the cleft are scarified and approximated with fine silk sutures placed one third of an inch apart. 


\section{Esophagotomy}

When a foreign body becomes so firmly lodged in the esophagus that it cannot be moved by the forceps or probang, the esopliagus must be opened and the foreign body extracted. If in the cervical region the procedure is as follows: Place patient in the dorsal position, with neck well extended. Then apply a local anesthetic of four-per-cent solution of cocain and adrenalin. Have at hand a razor, scalpel, artery forceps, tissue forceps, needles, catgut sutures, and silk sutures.

Technic.-The operative area is shaved and disinfected in the usual manner and the cocain solution injected subcutaneously.

An incision above the foreign body is made through the skin with the scalpel, and the esophagus is exposed by blunt dissection, the vessels and nerves being pushed aside. The esophagus is then incised and the foreign body extracted. Then the esophageal wound is closed with catgut sutures, first the mucous membrane and then the muscular and fibrous layers of the wall.

The skin incision is sutured with silk, drainage being provided for at the lower margin of the wound.

Water must be withheld for at least twenty-four hours and all food for four days, the patient being fed per rectum, after which well minced and sloppy food may be given in small quantities. Unless this part of the treatment is fully carried out, healing is interfered with, infection invariably takes place, and an esophageal fistula often results.

\section{Tracheotomy}

Tracheotomy, or incision into the trachea for the purpose of inserting a tube, is essentially an emer- 
gency operation in the dog to avert impending suffocation during attacks of laryngitis, foreign bodies in the pharynx or esophagus, and sometimes in cases where the nasal chambers become plugged with clotted blood or mucous.

Place the patient in the dorsal position, with neck fully extended, and apply a local anesthetic of cocain and adrenalin solution. The following instruments must be ready for use: scalpel, razor, rat-toothed forceps, tracheal retractor, and tracheotomy tube. Also have plenty of dressings and swabs at hand.

Technic.-After sliaving and disinfecting an area about three inches square immediately below the larynx, a median incision is made through the skin and muscles onto the trachea. The incision should begin about one inch below the larynx and extend backwards about one and one-half inches. Hemorrhage must be completely controlled before the next step of incising the trachea is taken. The trachea is then seized with the rat-toothed forceps and an incision made in its middle line, long enough to allow of the insertion of the tube. To facilitate this, the trachea wound should be dilated with the retractor, as otherwise considerable difficulty may be experienced in inserting the tube. The aftertreatment consists of removing the tube daily and cleaning it, cleaning the wound, and reinserting the tube. After permanent removal, the wound should be treated antiseptically and allowed to heal by granulation. 


\section{SECTION III}

\section{SURGERY OF THE EYE}

\section{Injuries}

TJURIES to the orbit are fairly frequent from 1 blows, kicks, collisions, and other causes, and may involve the surrounding soft tissues, bones, or contents. Treatment will vary naturally with the extent and particular nature of the injury, either coming under the head of general surgical principles or special operative considerations. One of the common results of severe traumatism of the orbital region is dislocation of the eyeball, which may or may not be a very serious condition, depending entirely upon the injury inflicted on the eye itself.

If the injury is recent, with no laceration of the globe or interocular. hemorrhage, there is every chance of the eye being saved and vision unimpaired. However, in cases where the globe is lacerated, cold, or shows evidence of interocular hemorrhage, enucleation is the only proper procedure.

In attempting to replace a dislocated eyeball, the chief difficulty encountered is the inversion of the eyelids and the swollen condition of the parts.

The simplest and most effectual procedure is as follows:

Place the patient in the abdominal position and induce a general anesthesia. Have ready for use a small scalpel, a small-pointed bistoury, dressing, swabs, sterile gauze, fine needles, silk sutures, bandage or eye cap, and collodion (all the instruments being sterilized by boiling).

Technic.-The skin around the outer canthus is 
shaved and disinfected and the eyeball carefully cleaned with warm, sterile, normal saline solution. An incision about half an inch in length is made through the skin at the outer canthus in the same direction as the palpebral fissure, thus enlarging it.

It is now possible to evert the lids, but ustally it is found impossible to return the eye until the orbital sheath, the edge of which can be felt as a tense band closely gripping the eyeball, is incised. This is done by slipping the probe-pointed bistoury between the globe and the sheath, turning the edge to the constriction and cautiously dividing it.

If the lids be now held apart and gentle pressure with the palm of the hand applied to the cornea, the eye will return quite easily to the orbit and the operation is complete.

The wound at the outer canthus is sutured, dried, and sealed with collodion, and a pad of gauze is placed over the eye and maintained in position by a bandage or eye cap.

The after-treatment consists in allaying any irritation, with soothing collyria.

\section{Warts}

Warts on the eyelids are quite common and should be removed by excision, never by ligation, as the irritation produced may lead to irreparable damage to the eye caused by the patient's endeavors to remove the offending ligature with his hind feet.

\section{Foreign Bodies}

Foreign bodies are a frequent source of trouble, the extent of which will vary according to the nature of the foreign bodies. These gain entrance to the conjunctival sac or impinge on the cornea and as a rule give rise to only temporary irritation, which quickly subsides upon their removal. Particles of 
metal, thorns, and other sharp objects, which penetrate the cornea, either partially or completely, are more dangerous, sometimes leading to ulceration and abscess formation which may cause destruction of the eye. Larger bodies may so lacerate the cornea as to cause evacuation of the aqueous humor and collapse of the eye, or may completely disrupt the whole ocular apparatus.

The removal of substances such as eyelashes, dust, small pieces of cinder, or metal lodged in the conjunctival sac, is best accomplished by first of all putting a drop or two of a four-per-cent cocain solution into the eye, to diminish sensibility, and then everting the lids, locating the foreign body and removing it gently with a loop of horsehair.

Pieces of metal that have stuck onto or into the cornea may be removed with fine forceps, the eye spud, or with a magnet (if steel or iron).

If the eye is destroyed, as is usually the case when injured by bullets or shotgun pellets, enucleation should be immediately performed.

Injuries to the eye by chemicals are not frequent, but when occurring are always serious.

Acids must be neutralized with alkali, and vice versa.

Lime is best rendered harmless by flooding the sac with a saturated solution of sugar, as sugar neutralizes the corrosive action of lime. The resulting irritation from any cause is to be allayed under the line of treatment laid down for the varying conditions produced.

\section{Entropion}

Entropion, or the turning in of either the upper or lower eyelid, is a congenital condition nearly always calling for surgical interference on account, of the great irritation produced by the eyelashes 
impinging on the cyeball. The object sought by the operation is the reduction of the superfluous skin of the cyelid and the restoration of the lid to its normal position.

Tlie following instruments and dressings are sterilized by boiling and laid in sterile water in sterile instrument trays: a scalpel, a pair of curved scissors, entropion forceps, dressing forceps, needle holder, six needles and sutures (silkworm gut), swabs, ether, and iodized collodion.

Place the patient in the abdominal position and induce a general anesthesia.

Technic.-The eyelid is shaved and cleaned in the usual manner, and a fold of skin parallel to the direction of the cyelid seized with the entropion forceps. Care must be taken to pick up skin only, and only just enough of it to bring the eyelid back to normal. The forceps are then clamped and the fold of skin excised with the scalpel or scissors.

The cut must be made close to the forceps. The result is an elliptical wound, which, when the hemorrhage has been controlled by pressure, is sutured by interrupted stitches placed one eighth of an inch apart. The wound is then swabbed with ether, which is allowed to evaporate, and finally sealed with iodized collodion. Sutures are to be removed in about six days.

If neatly executed, and if strict attention is paid to asepsis, most excellent results are obtained by this operation, and unless infected the wound produces no irritation and the patient shows no inclination to interfere with it. Should he do so, howevar, an Elizabethan collar should be placed on him to prevent him scratching it with his hind feet. .

In addition to the surgical treatment, any irritation of the conjunctiva or cornea must be treated with mild astringent and antiseptic collyria. 


\section{Ectropion}

Ectropion is the opposite condition to that described above, namely, the turning out of the eyelid, and gives the animal a most unsightly appearance. The object of operative measures is to restore the eyelid to its normal position by shortening the lid in its longitudinal direction. This is affecter by excising a triangle-shaped flap of skin from the affected lid, the base of the triangle being parallel to the edge of the lid. The technic is the same as for entropion, the size of the flap being determined by experimental manipulations of the skin of the lid.

\section{Pterygium}

Pterygium, or dermoid growth on the cornea, is generally a congenital condition and consists of an outgrowth of the conjunctiva, which covers more or less of the sclerotic and cornea. As a rule numerous hairs project from it and cause great irritation. In some cases the growth is firmly attached to the underlying tissues; in others it is attached only at its two extremities.

Operation consists in the careful dissecting off of the membrane, and is always advisable, owing to the irritation produced. The following instruments are sterilized and laid ready for use: a Graefe's scalpel, fine rat-toothed forceps, eye speculum, and swabs of absorbent cotton.

Place the patient in the abdominal position and induce a general anesthesia by H-M-C and chloroform. The operation can be performed with local anesthetics, but with increased risk of injury to the eye.

Technic.-The patient being completely under the influence of the anesthetic, the operator inserts the eye speculum and drops into the eye a few drops of adrenalin solution. While the assistant steadies 
the head, he then seizes the growth at its corneal attachment and with the Gracfe's scalpel proceeds to dissect it off. The blade of the knife must be kept parallel with the cornea to prevent injury, and moderate tension should be kept on the forceps until the growth is finally removed. Hemorrhage is easily controlled by adrenalin solution and mod. erate pressure.

The after-treatment consists in daily cleaning the eye with boric-acid solution, until all irritation has subsided.

\section{Displaced Orbital Gland}

In the dog, just beneath the membrana nictitans, is situated a little rounded mass of glandular tissue, the orbital gland. This gland, as the result of traumatism, may become displaced and appear at the inner canthus of the eye as a reddish and often angry-looking mass which is exceedingly unsightly and sometimes produces irritation. IVhile easily replaced, it is next to impossible to retain it in position, and surgical interference is the only means of removing this eyesore.

The instruments to be used are blunt-pointed scissors, rat-toothed forceps, and dressing forceps. Have ready also swabs, an adrenalin solution, and a four-per-cent cocain solution.

Place the patient in the abdominal position and apply a local anesthetic of four-per-cent cocain.

Technic.-After confining the patient in the abdominal position, a few drops of the cocain solution are placed in the lower conjunctival sac, which later on is flooded with adrenalin solution. When anesthesia is complete the assistant, who also steadies the patient's head, holds the membrana nictitans aside with the dressing forceps while the operator seizes the gland with the rat-toothed forceps and, 
pulling it out as far as possible, clips it away from its attachment with the blunt-pointed scissors. Hemorrhage is controlled by adrenalin and pressure, and the patient is released. The eye should be washed out daily for a week with boric-acid solution and the patient not exposed to direct sunshine, dust, flies, or strong winds until all signs of irritation have disappeared.

\section{Enucleation of the Eyeball}

This drastic procedure may be rendered necessary by varying causes, such as destruction of the eye by injuries, suppuration, or new growths invading the eye or orbit, or to relieve the patient of the persistent pain sometimes attending glaucoma.

Place the patient in the abdominal position and induce a general anesthesia by H-M-C and chloroform.

Have ready at hand a pair of curved scissors, a tissue forceps, two artery forceps, a rat-toothed forceps, and two needles and silk sutures, all the instruments to be sterilized by boiling and laid in sterile dishes filled with boiled water ready for use. Dressings, sterile swabs, gauze moistened with sterile glycerin, adrenalin chlorid solution, an eye dropper, an eye cap, and bandage must be ready for use.

Technic.-The patient being properly secured and completely under the influence of the anesthetic, the operator passes sutures through each eyelid, but does not tie them. The assistant keeps the lids apart by traction on the sutures during the whole of the following stages. The conjunctival mucous membrane is then seized with the forceps and buttonholed with the scissors, which are then introduced into the wound and the mucous membrance divided around the circumference of the orbit. The resulting 
hemorrhage is controlled quite easily by pressure and a few drops of adrenalin solution. The corned is then seized with the rat-toothed forceps and the eye pulled out as far as possible.

The muscles are then divided one by one with the scissors close to their sclerotic attachments, and finally the optic nerve is divided as far back in the orbit as possible. The hemorrhage is slight and easily controlled by pressure, ligation being not only unnecessary but harmful. The stumps of the muscles are then trimmed and shortened, and the orbit tamponed with the glycerinated gauze. The glycerin prevents the tampon adhering and renders its removal the next day easy and painless.

After firmly packing the orbit the lids are closed with the sutures already used in retracting them, and a sterile gatue pad is applied over the orbit and kept in position with an eye cap or bandage. The tampon is removed in twenty-four hours and the orbit swabbed out with sterile normal saline solution, taking care to remove all blood clots. The orbit is then repacked with the glycerinated gauze, care being taken to completely fill it so that the eyelids are prevented from turning in.

The orbit must be dressed after this manner every day until healing takes place, otherwise the lids will turn in from the contraction during the process of healing and cause great irritation, in addition to introducing infection.

If careful attention is given to asepsis, pus formation is either very slight or entirely absent. If, however, infection does occur, the orbit should be flushed out with a solution of chinosol at each dressing.

After healing is complete, a glass eye may be inserted or the patient may be made more presentable by the following procedure: 
Under complete anesthesia, and with all aseptic precautions, the margins of the lids are denuded of their skin and the lids sutured together (blepharorrhaphy). When union is complete and the sutures removed, melted surgical wax is introduced into the orbit by means of a hypodermic syringe, in sufficient quantity to produce the appearance of a normally closed eye. As the wax cools, it is gently kneaded to the proper form. 


\section{SECTIC N I V}

\section{SURGERY OF THE THORAX AND ABDOMEN}

\section{Paracentesis Thoracis}

THIS simple and harmless operation is performed I for the purpose of removing fluid from the thoracic cavity. It may be repeated with perfect safety as often as circumstances require, provided that rigid asepsis is maintained.

The patient must be in the standing position, but held firmly by an assistant. Apply a local anesthesia of ethyl chlorid spray, and have at hand a trocar and cannula, a razor, swabs, and iodized collodion.

Technic.-The point of puncture on either side is the intercostal space between the seventh and eighth rib. Shave and disinfect an area two inches square. Pull the skin backwards and introduce the sterilized trocar and cannula into the thoracic cavity, taking care to make the puncture midway between the ribs, thus avoiding the vessels and nerves. Withdraw the trocar, and allow the fluid to flow away.

A small trocar and cannula must be used, for otherwise the evacuation of the fluid is so rapid as to produce syncope and sometimes a fatal result. There is little danger of wounding the lung, as it is floated up and compressed by the fluid and is therefore well above the line of puncture.

In withdrawing the cannula, the fingers should press the skin down firmly on either side of it, to prevent its separating the skin from the muscles. It will be seen that after the cannula is withdrawn the puncture in the skin and that in the muscles 
and pleura do not coincide, thus averting the entrance of air through the wound. After withdrawal of the cannula the wound is swabbed dry and sealed with iodized collodion.

\section{Paracentesis Abdominis}

PUNCTURE of the abdominal cavity by means of
a trocar and cannula is necessary for the removal of the ascitic transudate in cases of abdominal ascites or the exudate of chronic peritonitis.

The operation is harmless provided surgical methods are observed, and may be repeated with perfect safety as often as circumstances require.

The patient must be in the standing position, held securely by an assistant and muzzled. For instruments use a razor, trocar and cannula, and probe, and for dressings have ready iodin, swabs, and iodized collodion.

Technic.-Shave and disinfect an area about two inches square just posterior to the umbilicus. At a point one inch behind the umbilicus introduce the sterilized trocar and cannula oblicpuely through the skin for one half an inch, then direct the trocar straight upward and press, until the loss of resistance indicates that the abdominal wall has been penetrated. Withdraw the trocar and allow the fluid to escape through the cannula. Should the flow cease, the cannula is probably blocked by the omentum or by a clot, and the probe should be inserted and the obstruction gently pushed away. In withdrawing the cannula the fingers should press against the skin as in paracentesis thoracis, and for the same reason. After withdrawal, the wound should be swabber with iodin and sealed with the iodized collodion.

\section{Paracentesis Vesicæ}

Puncture of the bladder is rendered necessary when that organ is dangerously distended with 
urine and where it is found impossible or inadvisable to pass the catheter. The operation is simple and harmless if proper surgical methods are used and should be performed without hesitation when conditions indicate it. The point of insertion of the fine trocar or hypodermic needle is directly anterior to the brim of the pelvis in the middle line in the bitch and just to one side of the penis in the dog. The operative area is shaved and disinfected as usual and the trocar, cannula, or hypodermic needle sterilized by boiling. The patient should be in the standing position, owing to the danger of rupturing the bladder during the struggling incident to being laid on the table.

\section{Laparotomy}

Laparotomy, or abdominal section, is the prelude to many surgical manipulations of the abdominal or pelvic organs. It is classed among the major operations, but thanks to, aseptic surgery it has now lost most of its former terrors and dangers.

The incision should be made in the linea alba in the long axis of the body, directly over the organ it is desired to remove or subsequently operate on. $\mathrm{By}$ the middle-line incision hemorrhage is avoided, the mammary glands in the case of the bitch are not interfered with, and the scar is scarcely noticed a month or so after operation. Complete surgical anesthesia is essential, otherwise the omentum or intestines are liable to protrude through the wound and seriously handicap the operator, besides increasing an hundred-fold the risk of that bête noir of abdominal surgery-infection, with its sequelic of peritonitis and death.

The animal should be in the dorsal position, then general anesthesia induced by H-M-C and chloroform. The instruments used, scalpel, razor, dress- 
ing forceps, artery forceps, and grooved dissector, should all be sterilized by boiling, and swabs, iodin, gauze and wool dressing, bandages, and boiled water be ready for use.

Technic.-Wash and shave the entire abdomen and disinfect as usual. With the scalpel make an incision the necessary length and exactly in the middle line through the skin, subcutis, and linea alba down to the peritoneum. Control hemorrhage. Incise the peritoneum, being careful not to wound the intestines or other organs, introduce the dissector, and sliding the back of the knife along the groove, slit the peritoneum open for the length of the wound required for further manipulation and proceed with whatever the abdominal section was performed for.

The wound should be closed with interrupted silk or silkworm-gut sutures, taking care to include all three layers of the abdominal wall; that is, the peritoneum, the muscles, and the skin.

The edges of the wound should be grasped with the dressing forceps while inserting the sutures, and care taken to prevent the latter from touching the body during inaction. Touch the wound only with sterilized instruments to avoid infection. When the sutures are all placed, tie them and swab off the wound with boiled water, then dry it with a sterile swab and apply the sterile gauze dressing and bandage.

Remove dressing in three days and inspect the wound. If it is dry and aseptic, dress again with sterile gauze and leave undisturbed for three days, when the sutures should be removed. If at the first inspection it is found that infection has occurred, the wound must be cleaned with hydrogen peroxid, dried, painted with tincture of iodin, and redressed with a sterile gauze dressing. The wound must then 
be dressed daily and the sutures removed in five or six days.

Diet.-Subsequent to laparotomy the diet must be wholly liquid-milk, beef tea, or beef juice. Raw or cooked meat may be allowed, but no bones or large pieces of meat, bread, or biscuit must be given for at least two weeks after the operation.

Nursing.-After the operation the patient should be confined in a perfectly clean kennel, be perfectly quiet, and be prevented from jumping, and ascending or descending stairs. A plentiful supply of clean, cold water should be constantly provided.

The temperature should be taken night and morning and recorded on a chart, as well as the condition of the bowels and urine.

\section{Gastrotomy}

Incision of the stomach in canine patients is chiefly performed for the removal of foreign bodies either in the stomach itself or in the lower part of the esophagus.

Preparation.-Perform laparotomy, the incision being made in the middle line, commencing about two inches posterior to the xiphoid cartilage and extending backward. The additional instruments and materials required will be a scalpel, long slender forceps, ten round milliner's needles, No. 8 or No. 9, threaded with No. 2 size black sewing silk, the thread being tied into the eyes. Also have ready a needle holder, gauze and a piece of rubber sheeting ten inches square, having a slit three inches long cut in the center.

Technic.-The stomach is located and pulled up through the slit in the rubber shecting, which is laid over the abdomen. The stomach is then carefully packed around with gatue so as to eliminate 
the possibility of any of its contents entering the abdominal cavity.

The foreign body is then pressed up against the stomach wall and an incision made directly over it and large enough for its removal. After the removal of the foreign body, the edges of the wound in the viscus are swabbed off with sterile water and sutured closely with the black sewing silk, using the Czerny-Lembert suture.* If the foreign body is located in the lower part of the esophagus the procedure is as follows: Make an incision into the stom-

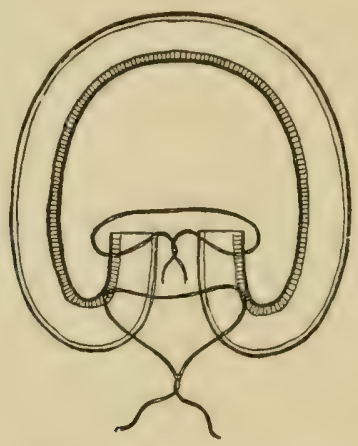

Czerny-Lembert Suture.

ach, introduce the long forceps as far as the foreign body, and extract it through the stomach wound, which is then sutured as before described. After suturing, the stomach is carefully cleaned oft and replaced in the abdominal cavity. The laparotomy wound is closed and treated in the same manner as described for that operation. Rectal feeding must be kept up for at least a week, and fluid food only allowed by the mouth for another two weeks after the operation.

*A combination of Czerny's and Lembert's sutures in circular enterorrhaphy; named for Vincent Czerny, a Heidelberg surgeon, born in 1S42, and Antoine Lembert (1S02-1S51), a French surgeon. 


\section{Enterotomy}

Incision of the intestines is performed for the purpose of removing foreign bodies or fecal accumulation.

Preparation.-Perform laparotomy, using the same instruments and materials listed and the same technic as for that operation and gastrotomy.

Technic.-The intestine with the foreign body is located and pulled up in the same manner and with the same precautions as is the stomach in gastrotomy.

Make an incision and extract the foreign body, clean up the bowel, and suture with Czerny-Lembert sutures, taking care to invaginate the edges of the bowel so as to bring the peritoneal surfaces together.

\section{Enterectomy and Intestinal Anastomosis}

In many cases of injury or gangrene of a portion of the intestines excision of a section of the bowel and anastomosis of the cut ends (enterorrhaphy) is often the only chance of saving the patient's life. To insure any chance of success, the operation must be performed before the onset of peritonitis and utter exhaustion of the patient.

Preparation.-Perform laparotomy, using the technic for that operation.

Have ready a scalpel, scissors, twelve No. 8 or No. 9 milliner's needles, twelve black sewing silk sutures. No. 2, tied into the eyes of the needles, a needle holder, six ligatures, No. 2 black sewing silk, two pairs of artery forceps, a pair of wire cutters, two hairpins, a piece of rubloer sheeting ten inches square with three-inch slit cut in center; gauze, swabs, and so on.

Needless to say, all the above must be perfectly 
sterile, and the utmost care taken to maintain asepsis throughout the entire proceeding:

Technic.-Dr. French's method, the simplest and the one giving the most satisfactory results, is as follows: The affected portion of the bowel is pulled up out of the wound and through the slit in the rubber sheeting, and surrounded with the sterile gauze. The operator must then carefully investigate the local blood supply and determine which mesenteric vessels can be ligated without danger of obliterating the blood supply to other than the part of the intestine to be removed. The branches that supply that portion are then ligated with fine. silk.

One prong of the hairpin is then passed through the mesentery at the upper point of resection, and both are brought transversely across the gut. The other pin is placed in the same manner at the lower point of resection. Both pins are clamped at their free extremities with artery forceps, thus completely obliterating the lumen of the bowel. The intestine is then severed with the scapel, quite close to the clamped prongs of the pin, the incision being extended to the mesentery so as to remove a wedgeshaped portion of it. The two pins are approximated and tied tightly together.

Halsted sutures are then placed, starting at the mesenteric attachment. Care must be taken that the margins are properly turned in so as to bring the peritoneal surfaces together. Next, the bowel is turned over and the other side sutured in the same manner. The pins are then untied and unclamped, severed at their bent ends with the wire cutters: and withdrawn, one prong at a time. The remaining four openings are closed with one stitch each. Finally the incision in the mesentery is closed by a continuous suture. 
Rectal feeding must be maintained for the first week. Then milk, beef tea, or very finely chopped meat for two weeks, after which the ordinary diet may be allowed.

\section{Ovariotomy and Ovariohysterectomy}

The objects of these operations are either to prevent pregnancy or to remove a diseased ovary or uterus. The most favorable age, where sterilization is the object, is from six to twelve months.

Preparation.-First periorm laparotomy as heretofore directed, making the incision just posterior to the umbilicus and extending backwards for about one ard one-half inches.

Technic.-Elevate the table so that the hind quarters are raised considerably. This allows the viscera to slide forward out of the operator's way. With the retractors (blunt aneurysm needles answer most admirably), pull the abdominal wound open, locate the cornua of the uterus by sight, and seize one of them with the forceps. Elevate the ovary by pulling on the horn, place a ligature of aseptic silk above and below the ovary, and excise with the scissors. Remove the other ovary by the same method, and close the laparotomy wound and dress as described previously. It will be noticed that at no stage of the operation are the operator's fingers introduced into the abdominal cavity, and that the intestines are not manipulated in any way.

In ovariohysterectomy, after locating the cornua, the uterus and ovaries are pulled up into the wound and the uterus ligated at the bifurcation, taking care to include the utero-ovarian arteries. Ligatures are then placed below each ovary, which are clipped away from their pedicles with the scissors. The broad ligament is then stripped away from the horns with the fingers and the uterus amputated anterior 
to the ligature. In this manner the ovaries and cornua are removed in their entirety, with practically no hemorrhage or risk of infection.

\section{Hysterotomy-Cesarean Section}

The object of this operation is the removal of live or dead fetuses. This operation, to be successful, must be performed sufficiently early and is preferable to, and offers better chances of recovery to the mother, than prolonged or forcible manipulation with the forceps.

Preparation.-Perform laparotomy, making the incision in the median line extending backwards from an inch behind the umbilicts to about an inch anterior to the brim of the pelvis. If the bladder is full, evacuate the urine by puncture with a sterile hypodermic needle or pass the catheter. Use the same instruments and materials as for gastrotomy.

Technic.-Bring the uterus well up into the wound, blocking it off from the peritoneal cavity with sterile gauze wrung out in warn, sterile, normal saline solution. Make an incision in the long axis of the horn and as close to the fundus as practicable, and extract the fetuses, all through the same incision.

Care must be taken not to incise or rupture the fetal membranes, as the risk of infection is thereby enormously increased. The best plan is for the operator to manipulate the fetuses into the uterine wound. Then the assistant extracts them and hands them as they are delivered to a second assistant, who looks after them. In this way the operator does not get his hands contaminated by coming in contact with the interuterine fluids. The after-births should then all be removed and the uterus swabbed out with normal saline solution. The uterine wound is sutured with No. 2 size black sewing silk, using 
the Czerny-Lembert or Halsted suture. The abdominal wound is closed in the usual manner, with interrupted sutures.

The dangers from this operation are infection, with all its attendant sequele and shock. These dangers are vastly increased if the fetuses are dead and decomposed. In cases where the fetuses are dead and the uterus shows signs of gangrene, or it is deciderl that the bitch is not to be used for breeding again, ovariohysterectomy should be performed in preference to hysterotomy.

The technic here differs slightly from a simple case of hysterectomy. The blood supply of the pregnant uterus is greatly in excess of the resting one, and the first.step is to ligate both utero-ovarian and uterine arteries. Ligatures are placed as for simple ovariohysterectomy, with the exception that the fundus of the uterus is ligated just behind the bifurcation and clamped about one inch posterior to the ligature.

The uterus is then divided between the ligature

UTERUS WITH CUT END

INVAGINATED \& SUTURED

and the clamp, the broad ligament broken dowtí and the cornua, with their contained fetuses, and the ovaries removed. The stump of the uterus is then carefully cleaned and invaginated and sutured with a purse-string suture. Unless this latter step is taken peritonitis almost invariably follows, as it is quite impossible to completely disinfect the uterine stump.

\section{Suprapubic Lithotomy}

The object of this operation is the removal of a vesical calculus. 
Preparation.-Perform laparutomy in the median line in the bitch, to one side of the penis in the dog, the incision extending forward from the brim of the pelvis.

Technic.-Bring the bladder up into the wound, incise it, and extract the calculus. Suture the vesical wound with two rows of Czerny-Lembert sutures, taking care not to suture the mucous membrane. Close the abdominal wound as in laparotomy. 


\section{OPERATIONS FOR HERNIA}

$\mathrm{H}$ ERNIA consists of the protrusion of an organ or portion of an organ out of its normal position, through an opening in the surrounding tissues. The varieties most commonly seen in the dog arc umbilical, ventral, and inguinal. Scrotal, femoral, and perineal hernias are much more rare.

The main principles of treatment are the same in all cases and consist in the return and retention of the organs to their normal position.

The instruments required include two scalpels, two pairs of artery forceps, two pairs of dressing forceps, scissors, probe-pointed bistoury, grooved dissector, needles and sutures, small trocar, and needle holder. Have ready also swabs, iodin, iodized collodion, gauze, dressings, and bandages.

Place the patient in the dorsal position and induce general anesthesia by the use of H-M-C and chloroform.

\section{Umbilical Hernia}

This condition consists of a swelling at the umbilicus. It may be soft, fluctuating, and easily reducible, or it may be irreducible because of ad. hesions or because the umbilical ring has contracted since the mass was extended. In the latter case it feels hard to the touch and contains only omentum.

It is dangerous because strangulation may occur and lead to fatal peritonitis.

Technic.-The shin is shaved and disinfected in the usual manner and the instruments sterilized. Make an incision over the center of the swellings 
through the skin only. Return the contents of the sac, being careful to break down all adhesions. Scarify the edges of the umbilical ring and draw them together with silk sutures. The skin is then sutured with silkworm gut or silk, the wound dried, and sealed with iodized collodion.

\section{Ventral Hernia}

Ventral hernia is usually the result of some injury that has lacerated or ruptured the subcuticular tissues, thus allowing some of the internal organs to protrude. It may also occur as a sequel to laparotomy, due to want of care in suturing.

Technic.-After the usual precautions have been taken for the maintenance of asepsis, make an in-cision through the skin down to the sac (peritoneal covering). Incise the sac, and return the contents to the abdomen, enlarging the hernial opening if necessary to effect this. Trim off the superfluous sac and after refreshing the edges of the muscles, place two rows of sutures. The deep row, of catgut, must include the peritoneum and muscular layers; the upper, of silk, closes the skin wound. After drying the wound, seal with collodion and, if necessary, support with a sterile pad of gauze and a bandage. In large rents of the abdominal wall, where there is risk of prolapses, a Bayer suture and dressing should be placed in addition to the simple suturing.

\section{Inguinal Hernia}

Inguinal hernia is more common in bitches than in dogs, and commoner in multipara than in maidens. As a rule the contents of the sac contain one or both cornua of the uterus, and frequently include intestine and omentum in addition.

Technic.-After thoroughly disinfecting the opera- 
tive area and inducing complete anesthesia, make an incision through the skin over the hernial sac, pick the sac up, with the forceps, and carefully incise it without wounding its contents. Break down any adhesions, and return contents to the abdomen. Seize the extremity of the sac with a pair of artery forceps and twist the sac-until it forms a sort of pedicle, and around this place an aseptic silk ligature. Cut off the lower portion of the sac, suture the external wound, dry, and seal with the iodized collodion.

- At times it is found necessary to enlarge the internal ring before reduction is possible. This must be done with great caution with the probe-pointed bistoury or proper hernia knife, always remembering that the external pudic vessels and nerves are situated toward the middle line and pass through the ring.

\section{Scrotal Hernia}

Scrotal hernia manifests itself as a soft, fluctuating, painless, and usually reducible swelling in the scrotum, the reduction being accompanied by a gurgling sound.

Technic-Disinfect the scrotum and anesthetize. Reduce the hernia, make an incision in the long axis of the scrotum down to the tunica vaginalis testis, and separate the latter from the surrounding tissues. Pull the testicle down and ligate the cord as high as possible above the ligature. Remove the testicle, leave the scrotal wound open, and treat antiseptically.

\section{Perineal Hernia}

This occurs as a soft swelling at the side of the anus. The herniated organs are usually omentum or bowel and the bladder.

In the latter case the condition is dangerous, as the bladder is twisted on itself and retention of trine 
a common sequence. It is often associated with neoplasms on the neck of the bladder, and enlargement of the prostate gland.

Operation.-Disinfect the area and anesthetize. If the bladder is herniated, evacuate the contents by puncture with fine trocar and cannula. Cut down on to the hernial sac and return the contents to the abdomen. Break down all adhesions, seize the sac with the forceps, twist into pedicle and ligate, and ablate remainder of sac.

Remove superfluous skin and suture with interrupted sutures, dry, and seal with collodion. As soon as the patient has recovered from the operation, perform laparotomy, replace the bladder (if this was the herniated organ), and stitch it to the abdominal wall with three sutures, which must penetrate only the muscular wall of the bladder.

\section{Femoral Hernia}

The technic for the operation on femoral hernia is the same as for inguinal hernia, but due regard must be paid to the proximity of the femoral vessels, since the hernia descends through the crural ring. 


\section{SECTION VI}

\section{SURGERY OF THE LEGS AND TAIL Sprains}

$S_{\text {PRAINS of the muscles and articulations are }}$ $S$ commonly the result of injury sustained by falling from a height, sudden turns, and injuries met with while jumping. The symptoms are pain, heat and swelling of the part affected, and complete or partial loss of function.

Treatment consists of enforced rest and, where possible, of immobilization of the injured member. To subdue the acute inflammatory condition, cold applications should be applied and frequently repeated; later hot fomentations and gentle massage should take the place of the cold packs and a mild liniment gently rubbed in or the parts painted with tincture of iodin.

\section{Dislocations}

Dislocations occur as the result of violent wrenching of a joint and are generally sustained while running, jumping, or falling from a height. The symptoms are swelling at the joint, shortening of the limb, and imperfect movement attended with great pain. The most common dislocations met with are those of the shoulder, elbow, stifle, and toe joints.

Since dislocations, wherever situated, can alway: be diagnosed by palpation and manipulation, and confirmed by the X-ray if necessary, only the principles of treatment will be given here and no space taken up with a consideration of any possible condition.

Treatment should be adopted as soon as possible 
after the injury has occurred. Place the patient under a general anesthetic to relax the muscles and so faciliate reduction. By palpation or, if available, by the $\mathrm{X}$-ray determine exactly the condition to be dealt with and then think out the manipulations required to reduce the joint to its normal position. When successful, the bones often go back into place with a distinct snap and the deformity of the joint disappears. After reduction, the whole extremity should be placed in a plaster bandage. When the dislocation occurs in the shoulder, hip, or stifle, where it is impossible to apply a bandage, the parts may be more or less immobilized by first of all clipping the hair, applying fish glue to the skin, and then pieces of book muslin, layer on layer, using the glue as frequently as required, until about a dozen thicknesses of the muslin have been applied. This glue cast sets very hard, is light, and supports the parts nicely. It is easily removed by soaking in warm water.

Dislocation of the tail is generally met with in greyhounds, whippets, or in dogs having a long tail. If seen sufficiently soon after injury the dislocation is easily reduced. The tail should be kept in place either by a glue bandage or adhesive plaster.

\section{Necrosis}

Necrosis of the caudal vertebra is frequently met with and in advanced cases necessitates amputation of the tail. The condition is the result of injury, such as a bite from another animal, or the tail being run over, jammed in a door, or trodden upon.

The symptoms of necrosis are a swelling in the affected region, tenderness and abscess formation. The treatment consists of free incision, the removal of sequestra, and free curettage. After operation 
the wound should be treated with tincture of iodin until healed.

\section{Amputation of the Tail}

Amputation of the tail may be performed by two methods: (1) circular incision through the skin muscles and vertebral joint; (2) the flap operation.

Amputation by Circular Incision.-Place the patient in the abdominal position, and have ready hair clippers, large scalpel, tape, gauze, bandage, and tincture of iodin. Clip the hair from the tail and paint with tincture of iodin. Pull the skin back toward the root of the tail and tie a piece of tape tightly round the tail, close to the body. Locate a joint, and with one cut of the scalpel sever skin muscles and articulation. Paint with iodin, apply a gauze dressing, and bandage. Remove the tape in eight hours and pull the skin down over the stump. Disinfect daily until healed. Healing occurs by granulation.

The Flap Operation.-This is the more surgical method and is to be preferred in old dogs and in the large breeds. Place the patient in the abdominal position and induce general anesthesia by H-M-C and chloroform. For instruments and materials have ready a scalpel, razor, scissors, artery forceps, needles and sutures, tape for tourniquet, gauze dressing, and bandage swabs, all to be sterilized by boiling.

Technic.-The tail is to be shaved and disinfected in the usual manner. Form two flaps by semielliptical incisions and dissect them arvay from the bone back to the next joint. Disarticulate the joint, pick up and twist the arteries, and suture the flaps together. Dress with gauze, and bandage: Remove the tourniquet in eight hours. 


\section{Fractures}

Fractures are termed simple when there is no external wound; compound, when the broken ends of the bone protrude through the skin; comminuted, when the bone is shattered into fragments; and complicated, when serious damage has been done to neighboring tissues, organs, vessels, or nerves. Greenstick fracture is the term applied to the bending and only partial fractures seen in young animals whose bones are more or less plastic. The symptoms of a fracture are pain, inability to support weight, and crepitus.

Reduction.-Besides being more humane, it renders the necessary manipulations simple and more effectual to reduce a fracture under the influence of a general anesthetic. An additional advantage of anesthetization, especially where $\mathrm{H}-\mathrm{M}-\mathrm{C}$ is used, is that the casts used to retain the fracture have time to harden before the patient comes out of the anes. thetic. Reduction is effected, and the ends of bone brought into apposition, by grasping the limb firmly with one hand, above the fracture, and bringing the lower portion into position with the other hand. The limb should then be bandaged, starting at and including the foot. The bandage is to prevent chafing and swelling from unequally divided pressure. A plaster of Paris bandage should now be rolled on the whole length of the limb, about three thicknesses being applied. The limb should be held straight until the cast dries, or a splint of cardboard, basswood, or poroplastic felt applied and bandaged on, and left there until the cast is hard.

With a compound fracture, the wound must first be thoroughly cleaned and disinfected, the fracture reduced, and a cast applied as in simple fracture, but a window must be left for access to and dress- 
ing of the wound. This window may be formed by placing a pill-box lid orer the wound when bandaging and applying the cast, afterwards removing it by cutting ont the parts above with scissors. The wound must be dressed every day and a dressing applied.

Fractures of the hip, shoulder, and pelvis are treated in the same way as described under dislocations. Limitation of exercise by confinement in a small kennel is the prime factor in the successful treatment of this class of fracture.

\section{Unfavorable terminations to be feared are:}

1. The limb may not be straight. This happens if the patient attempts to bear weight on it before the cast is properly hardened, or if the cast is removed too soon.

2. The formation of a false joint, imperfect uniois only taking place and ossification not complete.

3. Gangrene, owing to severe injury to the main blood supply or to pressure from too tight a bandage. A fetid smell from the bandaged leg and continual uneasiness and attempts at liching on the part of the patient give rise to suspicion of this condition and the bandages should at once be removed and the parts treated antiseptically.

4. Chafing of the tissues, from rubbing on the hard edges of the cast. This may be obviated by proper padding and rolling over the edges of the cast.

5. Septicemia, in the case of compound fractures.

\section{Amputation of Claws}

Amputation of claws is often rendered necessary owing to their becoming torn or injured. Where there is no bony attachment they may be simply snipped off with a pair of strong scissors, the resulting wound being afterwards treated antiseptically. 
When there is a distinct bony union, however, the parts must be shaved and disinfected, the skin incised, and the bone cut through with bone forceps. The wound is then sutured and sealed with iodized collodion.

\section{Interdigital Cysts}

These cysts cause considerable lameness and irritation. The symptoms shown are that the patient is continually licking or nibbling at the affected spot between the claws, and is more or less lame. Examination reveals redness of the part and a bleb. like swelling, which is tense and fluctuating, and generally painful to the touch.

Incise the cyst freely and destroy its walls by vigorous curettage with a sharp curette. Paint the affected part daily with tincture of iodin until healed.

\section{Tumors}

Tumors occur on any part of the body, and are classified according to their histology. Their description, varieties, and so on, belong entirely to the realm of pathology, therefore the reader is referred to the textbooks treating that special branch of medical science.

The only effectual treatment of tumors is excision, and since tumors vary in size, shape, and position, only general principles can be laid down for their removal. These are, maintain asepsis, ligate bloorl vessels immediately, regulate the skin so that no pockets are left, provide for drainage, and treat wound as usual. 


\section{SECTION VII}

\section{OBSTETRICS}

PARTURITION, and the diseases and accidents incident to the puerperal condition, belong to the general subject of obstetrics.

The period of gestation in the bitch is sixty-three days, or thereabout, and the act of parturition is usually accomplished without assistance; in fact, interference, except in the case of mal-presentations, unduly protracted labor, or some abnormal condition, is to be strictly avoided. The motto, "make haste slowly," is most applicable here, since even in a perfectly normal, healthy bitch labor may be extended over a considerable period of time. Unwarranted interference is always liable to be followed by disastrous results.

The symptoms of approaching parturition are considerable enlargement of the mamma and of the external genitals; a glairy discharge from the vulva, of which the mucous membrane, as well as that of the vagina, is of a purplish-red color. Urination is frequent and the bitch is restless, constantly seeking a place which to her is the most suitable and secluded in which to bring forth her young.

A large, roomy, clean, dry kennel, or for a small bitch a large box, should be provided and the bitch placed therein as soon as labor begins, otherwise she is liable to hide away in some more or less inaccessible place, rendering observation and any necessary help difficult or even impossible. As regards bedding, there is nothing better, more cleanly, or less liable to interfere with either the bitch, pups, 
or attendant, than a newspaper laid flat. It is easily removed and burned after parturition, and may then be advantageously replaced with hay, straw, or excelsior.

A box or basket lined with flannel must also be provided for the pups, which should be placed therein as soon as born, covered with a piece of flannel, and not returned to the mother until the last one has arrived and the kennel or box has been cleaned. They may then be placed with the bitch, which will in a normal case clean them and attend to them as her instinct prompts her.

In any case of parturition a cértain routine should be practiced, otherwise accidents or untoward sequelac are liable to result. After disinfecting the hands and cleaning the external genitals and vagina, a digital cxamination should be made and the following points noted,-the condition of the os uteri, the presentation of the fetus, the size of the pelvic opening, and the existence or otherwise of maternal malformation, new growths, or constrictions of the vagina.

The importance of this early examination for the discovery of abnormalities cannot be overestimated, since the attendant's whole mode of action depends upon it. For example, it is useless to expect a natural delivery if the pelvic outlet is excessively narrow, either by reason of congenital malformation or from a previous fracture, and a practitioner who allowed labor to proceed until the mother was exhausted and moribund before he discovered it would lay himself open to charge of malpractice. At least, he would lose his patient and his client. Too much stress cannot be laid upon the use of antiseptic precautions, both in making examination and in any manipulation required. The hand's must be disinfected thoroughly, the instruments must be sterilized 
by boiling (laying them in antiseptic solution for a few minutes will not sterilize them), and the hind quarters of the patient must be washed repeatedly with an antiseptic solution. The vagina and uterus in the parturient bitch are highly susceptible to" septic infection, and every effort must be cxerted to avoid infecting them.

In normal parturition the pups follow one another at irregular intervals varying from a few minutes to several hours, so that delay, unless the mother is exhausted, the fetus dead, or the pains continuous and without result, need not cause alarm or be thought to demand interference. If, however, the mother is exhausted or the pains weak or unproductive, an examination must be made, malpresentation corrected, if present, and stimulants-brandy or whisky combined with from one-fourth to one dram of fluid extract of ergot-given every half hour. Pituitrin has recently achieved great popularity as an agent for inducing contractions of the uterus in parturient animals. The dose is from one to four drams until delivery is effected or further measures required.

Let me here caution against undue haste in recourse to forceps, more bitches having been destroyed by the incautious use of forceps than there have been pups delivered with them. Remember always that the slightest laceration is an open door for the entrance of infectious germs, and that septicemia only too often follows such laceration.

The placenta is usually expelled soon after the birth of the fetus, the mother severing the cord with her teeth and, generally, eating placente and membranes. The placente should be counted, as retainerl placentx are a fruitful source of septic poisoning. After the birth of the last pup the bitch should be cleaned and given clean bedding, as heretofore de- 
scribed. She should then be given a hot meal, such as gruel, beef tea, or bread and milk, and should thereafter be supplied with a generous diet.

\section{Dystocia}

Impediments to delivery may arise from two sources, either maternal or fetal. The table below shows the kinds of dystocia that occur in each class:

\section{MATERNAL}

1. Malformations of the pelvis, congenital or traumatic.

2. Rigid pelvis.

3. Constrictions of the vagina.

4. New growths of cervix, os, or vagina.

5. Uterine inertia.

6. Hernia of the uterus.

7. Torsion of the uterus.

\section{FETAL}

1. Malformations.

2. Malpresentations.

3. Abnormal size.

4. Hydrocephalus.

5. Gaseous distension (dead fetus).

6. Engagement of two puppies simultaneously.

Dogs with elongated heads, such as greyhounds, spaniels, and collies, traverse the maternal passage with greater readiness than those with squarer, blunter heads, such as bull dogs, Boston terriers, and pugs, because with the latter there is not the gradual dilatation of the parts that takes place with the former class. However, although difficulty in delivery is often experienced with the short-nosed dogs, yet the conformation is normal and cannot therefore be rightly classed as fetal dystocia.

\section{Maternal Dystocia}

1. Pelvic malformation.-This may be congenital or the result of a previous fracture or of disease (rachitis). In either case it must be determined whether delivery per naturam viam can take place 
or not, and this must be decided at the onset of labor or after the preliminary examination. There are two courses open to the surgeon, one to endeavor to extract the fetus after crushing the head or performung embryotomy; the other, which gives by far the best results if done early enough, is cesarean section. This operation, if performed before the mother is moribund or septic infection has taken place, is justified by its success. The puppies in any event are saved, and the mother is given her only chance for life. It is as well to remove the ovaries at the time of cesarean section and thus effectually prevent a recurrence of the trouble.

2. Rigid pelvis.-In old bitches that have been kept from breeding until late in life relaxation of the pelvic ligaments and consequent dilatation does not take place as readily as in the young animal, hence difficulty in delivery is often experienced. The indications for management are the same as for the foregoing.

3. Constrictions of the vagina.-This condition arises from cicatricial contractions after injuries to the vaginal mucous membrane, usually incurred either during a previous parturition or as a result of operations for the removal of new growths or abscesses. It often is a serious and sometimes an insurmountable obstacle to delivery and may necessitate cesarean section. Before resorting to this, however, a cautious effort should be made to dilate the passage either with the fingers or with a trivalve speculum; or one may even try incising it, if ,its situation and extent warrant such a procedure. In many cases, however, the fetus may be extracted, after thorough lubrication with sterile vaselin or olive oil, by means of the forceps and traction cautiously applied.

4. New growths of the os, cervix, and vagina.- 
The most common new growths in the vagina are pedunculated fibromata and the infectious granulomata. If they are at all extensive they constitute a mechanical obstacle to delivery. New growths involving the os uteri and cervix are of rather rare occurrence, but when present are usually malignant. Cesarean section to save the puppies, with subsequent extirpation of the entire uterus or destruction of the bitch, is the only alternative.

Pedunculated tumors in the vagina should be removed with the écraseur and sessile tumors with the scalpel and curette, hemorrhage being controlled by pressure and styptics.

5. Uterine inertia.-This condition is one of the most common and at the same time the most vexatious with which the practitioner has to deal. It is most commonly found in highly bred, overly fat, pampered, or debilitated bitches. The uterine contractions become weak or entirely suspended after the birth of one or two puppies, sometimes even before the first one is delivered. It is the cause of great mortality, as the unborn puppies quickly die and decompose. The bitch must be given stimulantsbrandy or whisky orally and ergotin hypodermically, or fluid extract of ergot orally every thirty minutes until the contractions begin again.

If ergot fails, large doses of quinin sulphate combined with intrauterine injections of warm sterilized glycerin will often have the desired effect. One to four drams of pituitrin, repeated in one hour if necessary, is a remedy for this condition that gives much promise, but its usefulness is not yet positively decided. In any event, treatment must be persistent and continuous until the contractions are reëstablished. It is not only useless but bad practice to fish around with the forceps and try to remove the 
fetus by force unless it is well into the vagina. Such procedure only leads to lacerations or even perforations of the uterus.

6. Hernia of the uterus (one or both horns).-This is a most untoward complication of parturition. It occurs usually before labor commences. Attempts at reduction and ordinary delivery may be made, but cesarean section is usually the only solution of the difficulty.

7. Torsion of the uterus.-Twist of the uterus occurs but rarely and may be diagnosed by the futility of the uterine contractions and the imperviousness of the cervix. Sometimes the twist may be felt by digital examination. Failing to reduce the torsion by manipulation-that is, by fixing the os by the pressure of a finger or fixation forceps and rolling the bitch over in a direction opposite to that of the twist, cesarean section must be performed promptly.

It may seem that cesarean section is unduly emphasized and advocated in these pages, but the author's experience has been that, when performed early and skillfully executed, it holds out by far the best chance for life both to the mother and her young, and that blind endeavor with the forceps, with no room for a guiding finger, are far more fatal and much less justifiable. In fact, cesarean section, properly performed and at the right time, is a remarkably safe operation and should not occasion a higher mortality than simple ovariotomy.

\section{Fetal Dystocia}

No very definite rules can be laid down regarding fetal dystocia, since every case is a law unto itself.

1. Malformations.-In the case of deformed or malformed puppies it may be necessary to perform embryotomy before delivery can take place, or the 
fetus may be crushed with the forceps, in this way making possible its passage through the natural outlet. Extra large puppies, if the presentation is normal-that is; head, nose, and fore legs in line-often necessitate the use of traction either by means of the forceps or a snare. Great care in the selection of the forceps is necessary. Those with overlapping handles should be discarded on account of the danger of pinching and lacerating the vaginal mucous membrane. They should be adjusted well around the head, given a turu to make sure that only the fetus is grasped, and traction applied only during expulsive efforts of the mother.

2. Malpresentation.-The variations of malpresentations are legion and of such variety that a minute

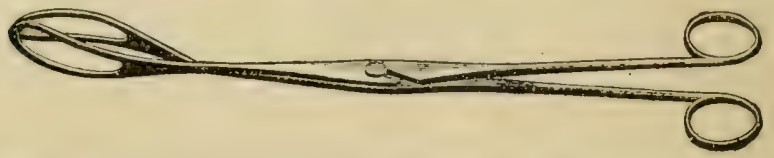

Bad Type of Parturition Forceps-Note the Overlapping Blades.

description of them would be liable to confuse the student and serve no good purpose. The main facts to remember are that the fetus can be delivered

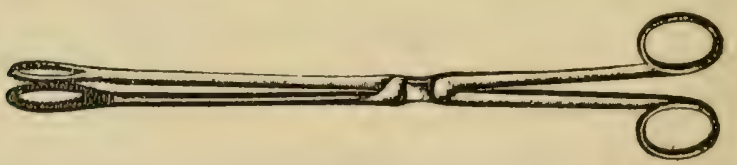

A Better Forcep, but Jaws Fatterned After No. 1 Are Preferable.

without embryotomy or cesarean section only if head, nose, and fore legs are in line, or if the hind legs and hind quarters are presenting. Therefore

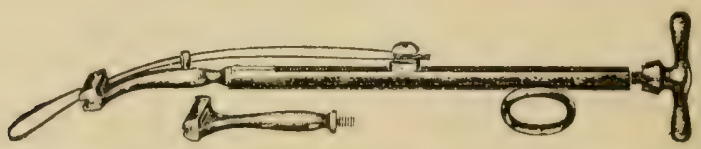

Wire Ecraseur-A Very Useful and Safe Instrument for Use In Embryotomy. 
every effort must be made to manipulate the fetus into one or the other of these two positions. Great patience and skillful manipulation with the finger are required and must be persisted in until either the desired result is attained or found to be impossible, when a decision between embryotomy and cesarean section must be made.

3. Abnormal size.

4. Hydrocephalus.-The abnormal size of the head must be reduced by puncture and draining with is trocar and cannula, followed by crushing with the forceps, when delivery cain usually be effected easily.

5. Gaseous distension.-Gaseous distension of the abdomen of the fetus should be reduced by puncture with the trocar and cannula.

6. Double engagement.-Two puppies presenting at the same time is a common occurrence, and is a source of great trouble to the practitioner, it being difficult to diagnose and still harder to rectify. Having recognized the condition by digital examination, the head of one puppy must be grasped with the forceps and traction exerted, the other puppy in the meanwhile being pushed back as far as possible witl a repeller.

\section{Accidents Liable to Occur During Parturition}

\section{Exhaustion}

In difficult and prolonged deliveries one or more of the puppies may die, or the bitch may gradually sink from exhaustion.

\section{Lacerations of the Vagina}

This accident isually occurs either from the incautious use of forceps or from the use of unsuitable forceps. The author has seen cases where complete rupture of the vagina had occurred and prolapse of the intestines taken place. These lacera- 
tions are always a grave source of danger from subsequent septic toxemia and must receive carefiul and thorough antiseptic treatment. Prolapse of the bladder, vide, may occur after rupture of the vagina.

\section{Rupture of the Uterus}

This serious accident is usually the result of too strenuous efforts to extract a dead fetus, the walls of the uterus having become weakened and friable from the solvent action of the products of decom-

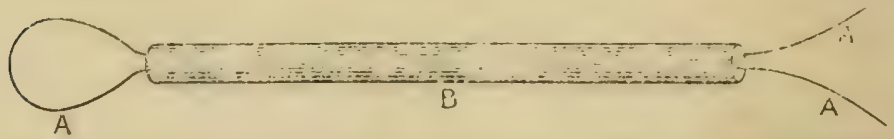

Snare for Extracting Puppies, Made Out of Flexible Copper Wire (A) and a Nickel-plated Thermometer Case (B). A Safe and Efficient Substitute for the Ordinary Forceps. Especially Useful in Small Bitches.

position of the dead puppy. The symptoms are vomiting, collapse, and a running down, weak pulse. The only chance for recovery lies in immediate laparotomy, suture of the rent in the uterus after it is properly cleaned, removing fetus and placenta, or total extirpation of the organ after double ligation. Otherwise septic peritonitis will prove rapidly fatal. Before closing the abdomen, the abdominal cavity must be irrigated with a decinormal saline solution. The prognosis is very unfavorable.

\section{Sequelæ to Parturition}

\section{Vaginitis}

Inflammation of the vagina is almost invariably the result of difficult labor, and is especially common in cases that have required prolonged manipulation and forceps delivery. It is indicated by a whitish, purulent discharge, sometimes with an extremely offensive odor. Upon examination of the vagina by means of a speculum the mucous membrane is found to be hyperemic, swollen, and cov- 
ered with a grayish-colored mucous exudate. Considerable tenderness may also be present and the examination resented by the patient, especially if the mucous membrane is extensively lacerated.

Treatment.-This consists of irrigations of antiseptics and astringents, such as solutions of chinosol 1-1000, one-per-cent potassium permanganate, oneper-cent sulphate of zinc, one-per-cent creolin, or the iodin saline solution mentioned elsewhere (normal saline solution, one pint; tincture of iodin, one dram).

\section{Prolapse of the Vagina and Uterus}

Of the two, prolapse of the vagina is most common. In neglected cases grave alterations, chiefly of a hypertrophic character, may take place in the vaginal mucous membrane, even gangrene sometimes resulting.

Symptoms.-The vagina protrudes through the top of the vulva and appears as a pear-shaped mass of inflamed tissue. In recent cases it appears moi.jt and covered with mucous; later it becomes drier and of a dark red or brown color.

In prolapse of the uterus, one horn only comes down and protrudes from the vulva as a mass of inflamed, mucous-covered tissue having salient borders.

Treatment.-Clean thoroughly with antiseptic solutions, and if much congestion is present constrict the blood ressels with local application of adrenalin solution, pushing back the prolapsed organ and taking care to restore the parts to their normal position. Otherwise straining will invariably take place. To overcome this, a dose of onc-fourth to one grain of morphin sulphate or one to three tablets (No. 1) of H-M-C should be given, and repeated as necessary. 
After the vagina has been replaced and smoothed out the lips of the vulva should be drawn together with two or three stitches, leaving only a sufficient opening for urination. The sutures may be left in three days or longer, as required to prevent further prolapse.

In prolapse of the uterus it is often almost an impossibility to completely smooth out the horn to its normal shape, even by means of some blunt, smooth instrument, and in such cases a recurrence of the prolapse may be looked for. Unless the uterus is completely replaced, straining will invariably persist and the condition recur. In these cases either one of two courses of surgical interference may be followed out: (1) laparotomy, reduction of the prolapse, and suture of prolapsing horn to the abdominal parietes, or (2) laparotomy and amputation of the offending horn, or total extirpation of the uterus.

The first procedure gives good results and should be practiced in cases where the uterus has suffered little or no injury from exposure or trauma. The second is indicated where grave changes have taken place in the uterus, and this is far preferable to the old operation of ligating and amputation, or amputation with the écraseur.

\section{Metritis}

Inflammation of the uterus is a common sequel to difficult, prolonged, or badly-handled labor, and is manifested in two forms: (1) simple metritis, which is a catarrhal inflammation of the mucous membrane, and (2) septic metritis, or puerperal fever, which is a very serious condition.

SIMPLE METRITIS

Symptoms.-The vulva is swollen and congested and there is a copious discharge of purulent, sanious, 
or sometimes putrid matter, increased in quantity after urination or defecation.

The animal draws attention to the hinder parts by constant licking, and the discharge may escape notice unless a careful examination is made. Slight constitutional disturbances occur; the appetite is capricious, and sometimes there is slight elevation of temperature. Unless treatment is prompt the condition is liable to become chronic.

Treatment.-Constitutional disturbances should be treated as they arise and the uterus and vagina flushed out daily with antiseptic, astringent solutions. Chinosol (1-1000) and one-per-cent potassium permanganate are among the best. The chronic form demands both local and constitutional treatment-one-per-cent solution of sulphate of zinc administered locally and iron tonics internally-to tone up the system. In very obstinate cases nuclein and the use of the staphylobacterins give good results and should be given a trial.

SEPTIC METRITIS-PUERPERAL FEVER

This is in reality a case of wound infection, and besides the intense inflammation of the uterus itself it is always accompanied by grave constitutional disturbances.

The parturient uterus is exceedingly susceptible to bacterial invasion and absorbs toxins readily, so that blood clots and remnants of a placenta which so freely decompose, are among the chief causes of this condition. As stated before, wounds and lacerations are of exceedingly grave import at this time, and readily become portals for the entrance of bacteria and their products into the circulation.

The intense inflammation of the mucous membrane extends to the deeper structures of the uterine walls, and often to the broad ligament and peri- 
toneum, when the case becomes complicated with a septic peritonitis. The constitutional symptoms are suppression of the mammary secretion, vomition, anorexia, great prostration, and weak, rapid pulse of a thready nature and gradually becoming imperceptible. The respirations are increased in frequency. The temperature at first is very high, but later on falls rapidly to subnormal in cases approaching a fatal termination. Local symptoms are pain on pressure to the abdomen, and copious, foulsmelling discharge from the vulva; the mucous membrane of the vulva and vagina is swollen, congested, and of a deep reddish-purple color. Mucous membrane of the mouth and lips is at first hyper-

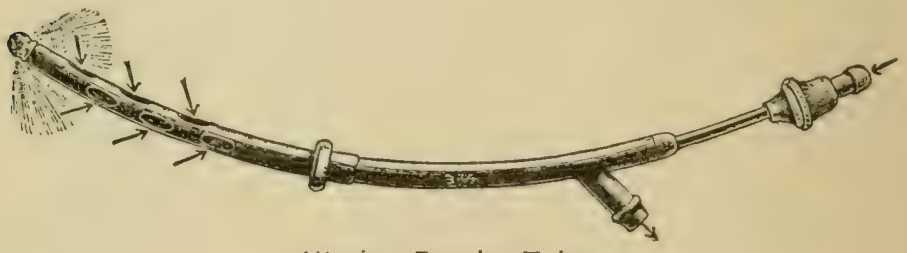

Uterine Douche Tube.

emic, but later of a livid color, and cold and clammy to the touch.

Acute cases run from twelve to twenty-four hours, and the prognosis is as a rule unfavorable.

Treatment.-To be successful, treatment must be instituted early and must be pushed energetically. Nuclein should be given from the outset, and administered three times daily until recovery, and the following given every three hours to effect: ergotin, quinin sulphate, iron phosphate, and fluid extract of echinacea, of each two grains.

To combat the great prostration, stimulants should be given, such as one dram of spirits of camphor, hypodermically every three hours.

The vagina and uterus must be irrigated out sevcral times a day, first with a sterile, warm, normal 
saline solution, until the solution runs out clear; then with antiseptic solutions of chinosol or potassium permanganate. After thoroughly irrigating as above described, the uterus should be filled with a hot, normal, saline solution and the patient allowed to retain it as long as possible.

Antistreptococci serum should be injected hypodermically, giving ten centimeters once or twice a day. Hot applications, such as antiphlogistin poultices, applied to the abdomen give relief from the abdominal pain. The patient should be kept warmly covered, and the diet restricted to beef tea, milk, or meat extracts.

The after-treatment consists of a generous diet and tonics, gentle exercise, and the avoidance of exposure to wet and cold.

\section{Mammitis}

Inflammation of the mamme and lacteal glands is of common occurrence in neglected bitches and in heavy milkers. It may vary from a slight congestion to intense inflammation with abscess formation, sloughing, and obliteration of portions of the glands.

Cause.-The causes are invasion of pyogenic bacteria, retention of milk (the result of taking the puppies away immediately after birth, or too early, or from their death), or from cold and wet, and lying on a cement floor or damp earth.

Symptoms.-Indications of mammitis are shown by tenseness and tenderness of the parts; the milk is curdled, sometimes blood-stained, and, if abscess formation has taken place, accompanied by pus. The constitutional symptoms, varying with the intensity of the inflammatory processes, are raised temperature, partial or complete anorexia, rigors, and constipation. It is highly important that these cases be taken in hand at once. 
Treatment.-First of all a purgative should be given, one to three grains of calomel or one or two compound cathartic pills, U. S. P. or B. P., and if necessary the pain may be controlled by one fourth grain of morphin sulphate or one tablet of $\mathrm{H}-\mathrm{M}-\mathrm{C}$ (No. 1). Give calcium sulphide in one-sixth-grain doses every half hour until the breath smells strongly of hydrogen sulphid.

The affected parts must be fomented with hot water and the mammac gently massaged and milked out, care being taken to entirely remove all the clotted or curded milk. The fomentations may be repeated or a hot antiphlogistin poultice applied, the massaging being repeated after removal of the poultice.

After the acute stage is passed, inunctions of warm camphorated oil are of service. Abscess formation must be treated by free incision and antiseptic treatment.

Neglected cases may lead to induration of portions of the mammary glands, leaving the so-called mammary tumors.

\section{Eclampsia}

Eclampsia is a tonic-clonic convulsive spasm which attacks suckling bitches generally about the second or third week after parturition, sometimes earlier. It usually attacks bitches that are heavy milkers and that have heavy litters of puppies. The bitch is generally an extra good mother, the puppies as a rule laying on flesh rapidly.

The cause of this trouble is still somewhat in dispute, but the prompt response to the eliminative treatment rather scems to indicate that the retention of attotoxins is the cause.

Symptoms.-An attack comes on suddenly, the symptoms at first not being very well marked. The 
patient becomes restless and anxious; the eyes stare and respirations are increased; the mucous membranes are injected; muscular twitchings make their appearance, and the patient falls down and is nnable to rise. The patient then lies on her side with stiffened legs, the muscles becoming hard and tense, quivering at intervals. Clonic convulsions of all the muscles occur, especially those of the extremities; the respiratory muscles are also largely involved, in consequence of which the respirations become quickened and labored; the mouth opens, and the tongue protrudes and appears greatly enlarged. All visible mucous membranes are cyanosed; the pulse is small, hard, and rapid, and the temperature is normal or subnormal. The eyes are staring and the expression anxious, but consciousness persists throughout.

The mamma become flaccid and the secretion of milk is suppressed. Rigidity of the extremities is one of the most prominent phenomena, the joints being flexed only with great difficulty. In untreated cases, coma supervenes before death.

Treatment.-Narcotic and antispasmodic agents are indicated and should be combined with eliminative treatment. The line of treatment giving the best result in the author's hands is as follows:

Give hypodermically three H-M-C tablets (No. 1) to a bitch the size of a fox terrier and five tablets (No. 1) to one of the size of a collie. Induce anesthesia with chloroform by the drop method, keeping it up until the H-M-C has taken effect. The patient being completely relaxed, give hypodermically one twentieth grain of veratrin hydrochlorid.

Repeat the H-M-C as often as necessary to control the spasms, always pushing it to effect regardless of dosage. A brisk purge should be given as soon as the patient is able to swallow, one to three grains 
of calomel or the compound cathartic pill, U. S. P. or B. P. After the attack, the puppies should be weaned immediately and the bitch's strength built up by a generous diet and a course of tonics. 


\section{INDEX}

A

AbDomen, operations on the, 205

surgery of the, 203

Abdominal dropsy, 77

Abscess of the cornea, 124

of the testicle, 103

Accidents during parturition, 233

Acne, 147

Acute Bronchitis, 17

endocarditis, 106

gastritis, 51

inflammation of the kidney, 82

nephritis, 82

Administration of medicines, the, 9

Alopecia, 146

Amputation of claws, 223

of the prolapsed rectum, 69

of the tail, 221

Anal glands, obstruction of the, 72

Anastomosis, intestinal, 209

Anemia of the brain, 115

Anesthesia, 181

general, 182

Aneurysms, 111

Ankylostomum trigonocephalum, 168

Anthrax, 160

Anus, Absence of, 72 diseases of the, 66

fistula of, 71

prolapsus of, 70

Apoplexy, 118

Arsenic poisoning, 173

Ascaris marginata, 166

Ascites, 77

Atresia ani, 72

Auditory canal, catarrh of the, 132
B

BANDAGE, description of Priessnitz, 48

Bismuth-paraffin paste, formula of, 72

Bladder, diseases of the, 90 eversion of the, 97 inflammation of the, 90 prolapse of the, 96 retroflexion of the, 95 stone in the, 94 tumors of the, 95

Blood, diseases of the, 111 parasites of the, 111

Blood Vessels, diseases of the, 105-111

parasites of the, 112

Brain, anemia of the, 115 emulsion for treatment of chorea, $\mathbf{1 5 3}$ inflammation of, 113

Bronchi and lungs, diseases of the, 17

Bronchitis, 17

acute, 17

chronic, 20

\section{C}

CALCULI of the bladder, 94 urethral, 98

Canker of the ear, 132

Carbolic acid (phenol) poisoning, 178

Cardiac hypertrophy, 109

Caries dentum, 45 of the teeth, 45

Cataract, 128

Catarrh of the auditory canal, $\perp 32$

intestinal, 55

Catarrhal jaundice, 74

pneumonia, lobular, 25

Catarrh, simple nasal, 12

Cerebral hemorrhage, $\mathbf{1 1 5}$

Cerebrospinal meningitis, 117 
Cesarean section, 212

Choking, 48

Chorea, 151

brain emulsion for treatment of, 153

Chronic bronchitis, 20

endocarditis, 107

gastritis, 52

laryngitis, 15

nephritis, 84

Cirrhosis of the liver, 76

Claws, amputation of, 223

Cleft palate, harelip and, 43 operation for, 191

Cold in the head, 12

Colic, 56

Congenital opacities of the cornea, 127

Conjunctiva, information of the, 122

Conjunctivitis, 122

Constipation, 60

Constitutional diseases, infectious, 148

Cornea, abscess of the, 124 congenital opacities of the, 127

opacities of the, 126

Coryza, 12

Croupous pneumonia, 21

Crystalline lens, opacity of the, 128

Curette, French's rectal, 61

Cystitis, 90

prescriptions for, treatment of, 92

Cysts, interdigital, 224

Czerny-Lembert suture, 208

DEAFNESS, 133

Degenerations of the kidney, 87

Demodectic mange, 136

Dental formula, 43

Dermatitis, parasitic, 140

Diabetes insipidus, 88 mellitus, 80

mellitus, prescription for treatment of, 81

Diarrhea, 58

prescriptions for treatment of, 59

Dilation of the heart, 109
Diseases, infectious constitu. tional, 148

of the bladder, 90

of the blood and blood vessels, 111

of the bronchi and lungs, 17

of the ear, 131

of the eye, 122

of the heart and bloou vessels, 105

of the kidney, 82

of the liver, 74

of the mouth, pharynx and esophagus, 37

of the nervous system, 113

of the pleuræ, 31

of the prostate, urethra and prepuce, 98

of the rectum and anus, 66

of the retina, 129

of the skin, 135

of the stomach and in. testines, 50

of the testicles and scrotum, 103

of the upper respiratory passages, 12

venereal, 162

Dislocations, 219

Displaced orbital gland, 199

Distemper, 148

prescription for, 153

Dobell's solution, formula of, 47

Donovan's solution, formula of, 153

Dover's powder, formula of, 56

Dropsy, abdominal, 77 prescription for the treatment of, 79

of the thoracic cavity, 34

Dumb rabies, 158

Dystocia, 228

due to abnormal size, 233

due to constrictions of the vagina, 229

due to double engagement, 233

due to gaseous distension, 233 
Dystocia-

due to hernia, 231

due to hydrocephalus, 233

due to malformations, 231

due to malpresentation, 232

due to tumors of the os, cervix and vagina, 229 due to pelvic malformation, 228

due to rigid pelvis, 229

due to torsion of the uterus, 231

due to uterine inertia, 230

fetaı, 231

maternal, 228

\section{E}

EAr, canker of the, 132 diseases of the, 131

Earflap, hematoma of the, 188 ulceration of the, 131

Ears, operation for split, 189

Eclampsia, 240

Ectropion, 198

Eczema, 142

Emollient, formula of, 138

Empyema, superior maxillary sinus, 46

Encephalitis, 113

Endocarditis, acute, 106 chronic, 107

Enterectomy, 209

Enteritis, 63

Enterotomy, 209

Entropion, 196

Enucleation of the eyeball, 200

Epilepsy, 119

Epistaxis, 13

Epulis, 41

Erythema, 141

Esophagotomy, 192

Esophagus, diseases of the, 37 foreign bodies in the, 48

Eversion of the bladder, 97

Examination of patient, 7

Exhaustion due to parturition, 233

Extremities, necrosis of the, 220

tumor's of the, 220

Eye, diseases of the, 122
Eyeball, enucleation of the, 200

foreign bodies in, 195

surgery of the, 194

surgical injuries of the, 194

Eyelids, warts on the, 195

\section{$\mathrm{F}$}

FAVUS, 138

Femoral hernia, 218

Fetal dystocia, 231

Filari immitis, 111

Fistula in ano, 71

Follicular mange, 136

Forceps, obstetric, 232

Foreign bodies in the esophagus, 48

in the eye, 195

in the stomach, 53

Formula, dental, 43

for the treatment of stomatitis, 38

of bismuth-paraffin paste, 72

of Dobell's solution, 47

of Donovan's solution, 153

of Dover's powder, 56

of emollient, 138

of Lassar's Paste, 138

of Lugol's solution, 40

Fractures, 222

Furious rabies, 156

GAstritis, 51

acute, 51

chronic, 52

prescriptions for the treatment of, 53

Gastrotomy, 207

General anesthesia, 182

remarks, 7

Glans, tumors of the, 102

Glaucoma, 129

Glossitis, 40

Granuloma, 162

$\mathrm{H}$

HANDS, preparation of the operators, 186

Harelip and cleft palate, 43 operation for, 190

Head, cold in the, 12 surgery of the, 188 
Heart, diseases of the, 105 hypertrophy and dilation of the, 109 rupture of the, 110 valvular defects of the, 107

Hematogenous icterus, 75

Hematoma of the earflap, 188

Hamatozoa, 111

Hemiplegia, 116

Hemorrhage, cerebral, 115

Hemorrhoids, 66

Hepatitis, 76 interstitial, 76

Heptogenous Icterus, 75

Hernia, femoral, 218 inguinal, 216 operations for, 215 perineal, 217 scrotal, 217 umbilical, 215 ventral, 216

Hook Worms, 168

Hydrocyanic acid poisoning, 177

Hydrothroax, 34

Hypertrophy and dilatation of the heart, 109

cardiac, 109

of the prostate, 100

Hypodermic administration of medicines, 10

Hysterotomy, 212

ICTERUS, 74

hematogenous, 75

heptogenous, 75

Impaction of the stomach, 50

Infectious constitutional diseases, 148

Inflammation of the bladder, 90

of the brain, 113

of the conjunctiva, 122

of the kidney, acute, 82

of the liver, 76

of the parotid glandmumps, 47

of the pelvis of the kidney, 87

of the pleura, 31

of the spinal cord; 117 of the testicles, 103

of the throat, 46

of the urethra, 98

of the uterus, 236

of the vagina, 234

Inguinal hernia, 216

Injurles of the eye, surgical, 194

Instruments, preparation of, 185

Interdigital cysts, 224

Internal parasites and parasitisms, 165

Interstitial hepatitis, 76

Intestinal anastomosis, 209 catarrh, 55

Intestines, diseases of the, 50 surgery of the, 209 tumors of the, 65

Intravenous administration of medicines, 10

Intussusception, 57

Iodoform poisoning, 179

Iritis, 127

JAUNDICE, 74

catarrhal, 74

malignant malarial, $7 \mathrm{~b}$

\section{$\mathrm{K}$}

KenAtitis, 123

interstitialis, 124

superficialis, 123

suppurative, 124

Kidney, acute inflammation of the, 82

diseases of the, 82

inflammation of the pelvis of the, 87

LACERATIONS OF THE VAGINA, 233

Laparotomy, 205

Laryngitis, 14

chronic, 15

Lassar's paste, formula of, 138

Legs, surgery of the, 219

Lithiasis, 94

Lithotomy, suprapubic, 213

Liver, cirrhosis of the, 76

diseases of the, 74

inflammation of the, 76 
Lobar (croupous) pneumonia, 21

Lobular (catarrhal) pneumo. nia, 25

Lugol's solution, formula of, 40

Lungs, diseases of the bronchi and, 17

\section{M}

MALARIAL JAUNDICE, malignant, 75

Mammitis, 239

Mange, demodectic, 136 follicular, 136 prescription for, 136 sarcoptic, 135

Marginata, ascaris, 166

Mastitis, 239

Taternal dystocia, 228

Maxillary sinus, empyema of the superior, 46

Medicines, the administration of, 9

Meningitis, cerebrospinal, 117 spinalis, 117

Metritis, 236 septic, 237

Monoplegia, 116

Mouth, diseases of the, 37

Mumps, 47

Myelitis, 117

\section{N}

Nasal CatarrH, simple, 12

Neck, surgery of the, 188

Necrosis of the extremities, 220

Necrotic stomatitis, 38

Nephritis, acute, 82 chronic, 84 suppurative, 86

Nervous system, diseases of the, 113

Obstetric Forceps, 232

Obstetrics, 225

Obstruction of the anal glands, 72

Opacities of the cornea, 126 of the cornea, congenital, 127

Opacity of the crystalline lens, 128
Operation for cleft palate, 191

for harelip, 190

for split ears, plastic, 189

Operations on the abdomen, 205

for hernia, 215

Operative area, preparatory treatment of the, 185

Operator's hands, preparation of the, 186

Ophthalmia, periodical, 129

Oral administration of medicines, 9

Orbital gland, displaced, 199

Orchitis, 103

Ovariohysterectomy, 211

Ovariotomy, 211

Oxyuris vermicularis, 167

P
P.late, cleft, 43

operation for cleft, 191

Papilloma, 42

Paracentesis abdominis, 204 thoracis, 203

versicæ, 204

Paralysis of the sphincter vesicæ, 93

Paraphimosis, 101

Parasites and parasitisms, internal, 165

intestinal, 166

of the blood and blood vessels, 111

of the kidney, 88

Parasitic dermatitis, 140

Parotid gland, inflammation of the, 47

Parotitis, 47

Parturition, accidents during, 233 sequelæ to, 234

Pericarditis, 105

Perineal hernia, 217

Periodical ophthalmia, 129

Pharyngitis, 46

Pharynx, diseases of the, 37

Phimosis, 101

Phosphorus poisoning, 174

Piles, 66

Pin Worms, 167

Plastic operation for split ears, 189 
Pleura, inflammation of the, 31

Pleuræ, diseases of the, 31

Pleurisy, 31

Pleuritis, 31

Pleurodynia, 35

Pneumonia, 21

lobar (croupous), 21

lobular (catarrhal), 25

prescription for treatment of, 30

Poisoning, arsenical, 173 carbolic acid (phenol), 178

hydrocyanic acid, 177

iodoform, 179

pathology of, 171

phosphorus, 174

potassium cyanid, 177

strychnin, 175

Poisons and poisoning, 171

Preliminaries, surgical, 180

Preparation of instruments, 185

of the operator's hands, 186

Preparatory treatment of the operative area, 185

Prepuce, diseases of the, 98 tumors of the, 102

Prescription for distemper, 153

for mange, 136

for ringworm, 139

for the "treatment of dropsy, 79

for treatment of pneumonia, 30

for vermifuge, 167

Prescriptions for treatment of cystitis, 92

for treatment of diarrhea, 59

for the treatment of gastritis, 53

Priessnitz bandage, description of, 48

Prolapse of the bladder, 96

Prolapsed rectum, amputation of the, 69

Prolapse of the vagina and uterus, 235

Prolapsus ani, 70

recti, 67
Prostate, diseases of the, 98 hypertrophy of the, 100 tumors of the, 101

Prostatitis, 99

Pterygium, 198

Puerperal fever, 237

Puppy snare, 234

Pyelitis, 87

Q

QUININE treatment for rabies, 158

RABIES, 155

dumb, 158

furious, 156

quinine treatment for, 158

serum treatment for, 158

Ranula, 41

Rectal administration of medicines, 11

Rectum, amputation of the prolapsed, 69

diseases of the, 66

prolapsus of, 67

Respiratory passages, diseases of the upper, 12

Retina, diseases of the, 129

Retroflexion of the bladđer, 95

Rhinitis, 12

Ringworm, 138 prescription for, 139

Round Worms, 166

Rupture of the heart, 110 of the uterus, 234

\section{$\mathrm{S}$}

Sarcoptic Mange, 135

Scrotal hernia, 217

Scrotum, diseases of the, 103 tumors of the, $\mathbf{1 0 4}$

Septic metritis, 237

Sequelæ to parturition, 234

Serum treatment for rabies, 158

Sheele's acid, 184

Sinus, empyema of the superior maxillary, 46

Skin, diseases of the, 135

Snare, puppy, 234

Sore throat, 46

Spasm of the sphincter vesicæ, 92

Spaying, 211 
Sphincter vesicæ, paralysis of the, 93 spasm of the, 92

Spinal cord, inflammation of the, 117

Spiroptera sanguinolenta, 112-166

Split ears, plastic operation for, 189

Sprains, 219

Stomach, diseases of the, 50 foreign bodies in the, 53 impaction of the, 50

Stomatitis, 37 formula for the treatment of, 38

necrotic, 38

ulcerosa, 38

Stone in the bladder, 94

strongylus vasorum, 112

Strychnin poisoning, 175

Supperative keratitis, 124 nephritis, 86

Suprapubic lithotomy, 213

Surgery of the eye, 194 of the head and neck, 188 of the intestines, 209 of the legs and tail, 219 of the thorax and abdomen, 203

Surgical preliminaries, 180

Suture, Czerny-Lembert, 208

T.FNA, 169

$$
\mathrm{T}
$$

Tail, amputation of the, 221 surgery of the, 219

Tapeworms, 169

Tartar, 44

Teeth, the, 43 caries of the, 45

Testicle, abscess of the, 103

Testicles, diseases of the, 103 inflammation of the, 103 tumors of the, 104

Tetanus, 158

Thoracic cavity, dropsy of the, 34

Thorax, surgery of the, 203

Thread worms, 166
Throat, inflammation of the, 46

Tracheotomy, 192

Treatment of the operative area, preparatory, 185

Tricocephalus depressiusculus, 169

Tuberculosis, 161

Tumors of the bladder, 95

of the extremities, 220

of the glans, prepuce, and vagina, 102

of the intestines, 65

of the kidney, 87

of the mouth, 42

of the prostate, 101

of the testicles and scrotum, 104

Typhlitis, 62 $U$
UICERATION of the earflap,
131.

Umbilical hernia, 215

Urethra, diseases of the, 98

Urethral calculi, 98

Urethritis, 98

Urticaria, 146

Uterine douche tube, 238

Uterus, inflammation of the, 236

prolapse of the, 235

rupture of the, 234

\section{V}

VAGINA, inflammation of the, 234

lacerations of the, 233

prolapse of the, 235

tumors of the, 102

Vaginitis, 234

Valvular defects of the heart, 107

Venereal disease, 162

Ventral hernia, 216

Vermifuge, prescription for, 167

\section{W}

WARTs in the mouth, 42

on the eyelids, 195 


$=$ 




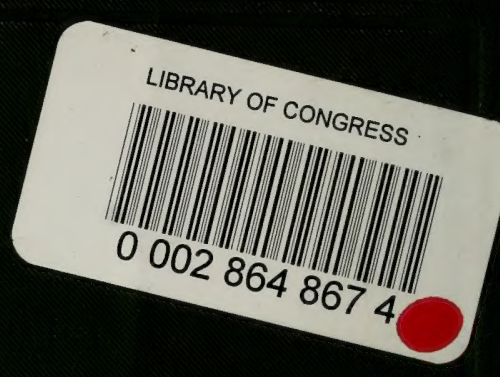

